ENG. 20040713.0003

QA: QA
000-30R-WIS0-00100-000-002

July 2004

BECHTEL

SAIC COMPANYLC

\title{
Waste Package Component Design Methodology Report
}

Edited By:

Delwin C. Mecham

Prepared for:

U.S. Department of Energy

Office of Civilian Radioactive Waste Management

Office of Repository Development

1551 Hillshire Drive

Las Vegas, Nevada 89134-6321

Prepared by:

Bechtel SAIC Company, LLC

1180 Town Center Drive

Las Vegas, Nevada 89144

Under Contract Number

DE-AC28-01RW12101 


\section{DISCLAIMER}

This report was prepared as an account of work sponsored by an agency of the United States Government. Neither the United States Government nor any agency thereof, nor any of their employees, nor any of their contractors, subcontractors or their employees, makes any warranty, express or implied, or assumes any legal liability or responsibility for the accuracy, completeness, or any third party's use or the results of such use of any information, apparatus, product, or process disclosed, or represents that its use would not infringe privately owned rights. Reference herein to any specific commercial product, process, or service by trade name, trademark, manufacturer, or otherwise, does not necessarily constitute or imply its endorsement, recommendation, or favoring by the United States Government or any agency thereof or its contractors or subcontractors. The views and opinions of authors expressed herein do not necessarily state or reflect those of the United States Government or any agency thereof. 
Waste Package Component Design Methodology Report

Originator:

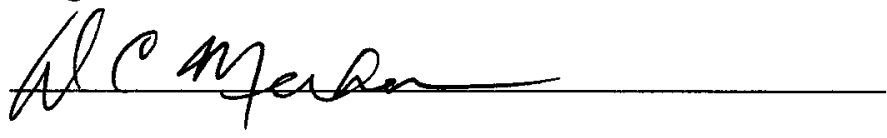

D.C. Mecham, Editor and Thermal Lead

Checkers:

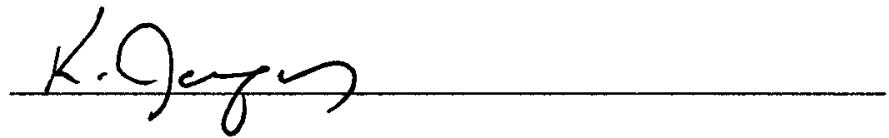

K. Jaquay, Structural

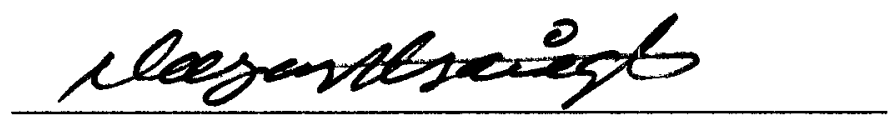

N.T. Alsaeigh, Thermal and Lead Checker

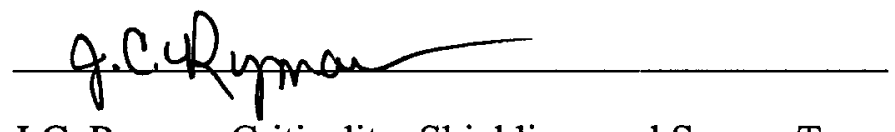

J.C. Ryman, Criticality, Shielding, and Source Term

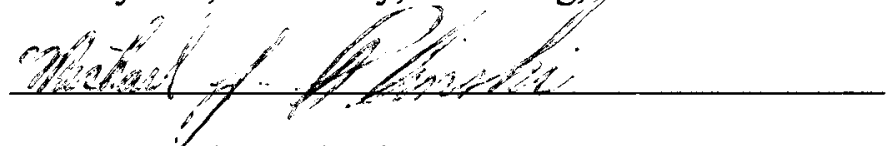

M. J. Plinski, Fabrication

Quality Engineering Representative:

$\frac{\text { Dawies divicy }}{1}$

D. J. Tunney

Sr. Quality Assurance Specialist

Responsible Manager:

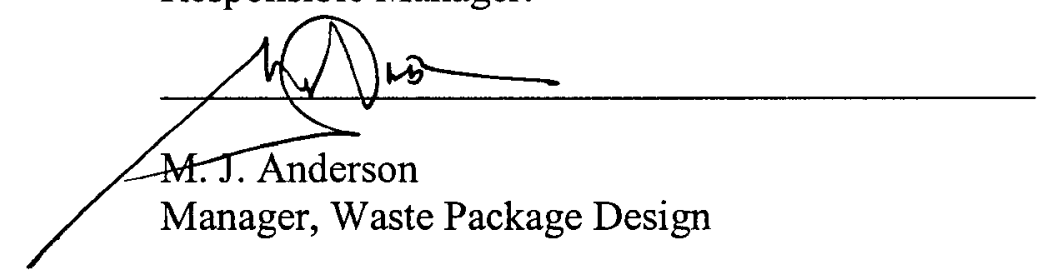

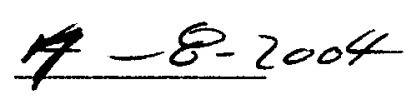

Date

Date

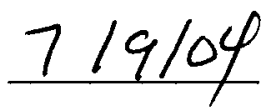

Date

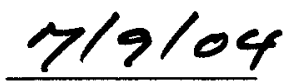

Date

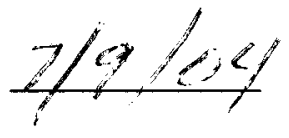

Date

$7 / 9 / 2004$

Date

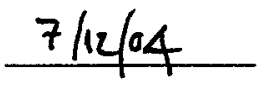

Date 
INTENTIONALLY LEFT BLANK 


\section{CHANGE HISTORY}

\begin{tabular}{|c|c|c|c|}
\hline $\begin{array}{l}\text { Revision } \\
\text { Number }\end{array}$ & $\begin{array}{l}\text { Interim } \\
\text { Change No. }\end{array}$ & $\begin{array}{c}\text { Effective } \\
\text { Date }\end{array}$ & Description of Change \\
\hline 00 & -- & $5 / 16 / 2000$ & $\begin{array}{l}\text { Initial issue of ANL-EBS-MD-000053 REV 00, } \\
\text { Waste Package Design Methodology Report, } \\
\text { MOL.20000526.0335. }\end{array}$ \\
\hline 01 & -- & $9 / 28 / 2001$ & $\begin{array}{l}\text { Initial issue. Supersedes ANL-EBS-MD-000053 } \\
\text { REV 00, Waste Package Design Methodology } \\
\text { Report, 5/16/2000, MOL. 20000526.0335. } \\
\text { Modified to reflect changes in waste package } \\
\text { design and updates to the design methodology } \\
\text { used in the development and verification of } \\
\text { design processes. }\end{array}$ \\
\hline 02 & -- & $3 / 19 / 2002$ & $\begin{array}{l}\text { Modified to reflect changes in waste package } \\
\text { design and updates to the design methodology } \\
\text { used in the development and verification of } \\
\text { design processes. }\end{array}$ \\
\hline
\end{tabular}

History of TDR-MGR-MD-000006 is provided above

000

-- NA

001 $-\quad$ NA

002

NA
Extensive revision to reflect design changes and updates to design methodology during transition from Site Recommendation to License Application effort. Revised DI number from TDR-MGR-MD-000006 to 000-30R-WIS000100-000-000. Changed name of report from Waste Package Design Methodology Report to Waste Package Component Design Methodology Report

Minor revisions marked by change bars: updated references, deleted use of ACUSOLVE, clarified use of $B \& W$ fuel as representative of other PWR fuels in response to Condition Report 2261, improved description of criticality analysis, converted DIRS to new format, added structural failure criteria.

Several editorial changes.

Updated reference in 4.1.1 Added event sequence in 6.2.2.5. Typographical errors corrected in tiered failure criteria, section 6.2.4. 
INTENTIONALLY LEFT BLANK 


\section{CONTENTS}

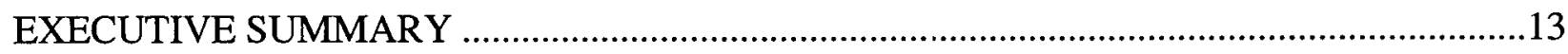

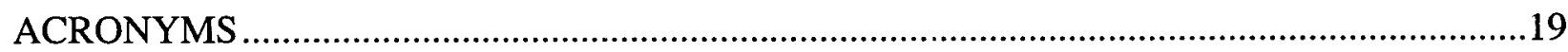

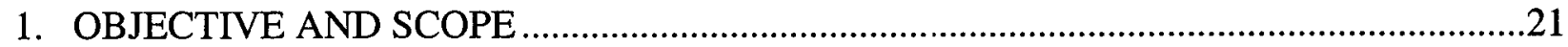

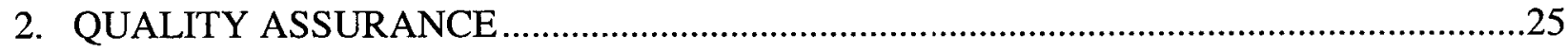

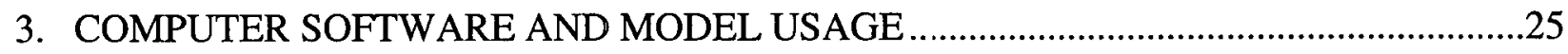

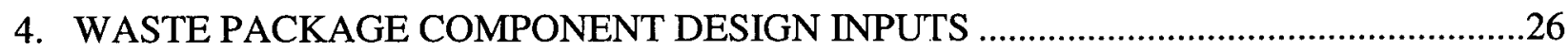

4.1 REQUIREMENTS .............................................................................................. 26

4.1.1 Assigned Requirements ...............................................................................27

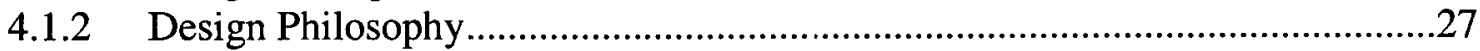

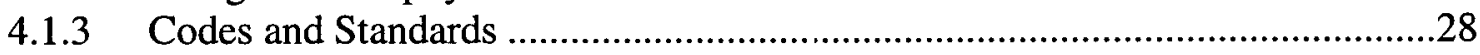

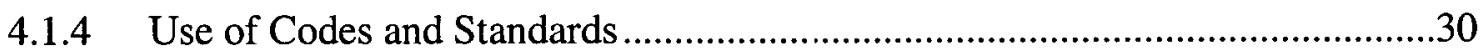

4.2 INFORMATION AND PARAMETERS ………...................................................... 34

4.2.1 Source Term ...........................................................................................34

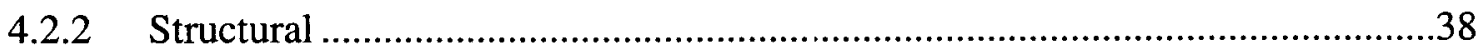

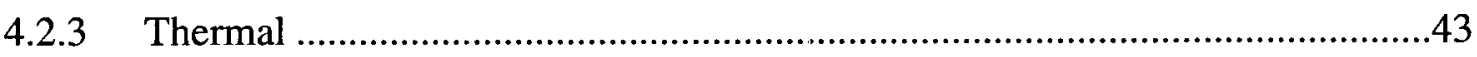

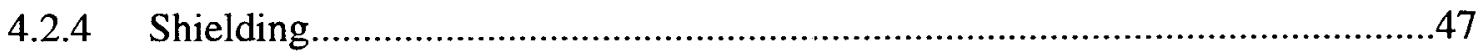

4.2.5 Criticality Analyses .........................................................................................50

4.3 INTERFACE WITH OTHER PROJECT ORGA.NIZATIONS ………………............. 50

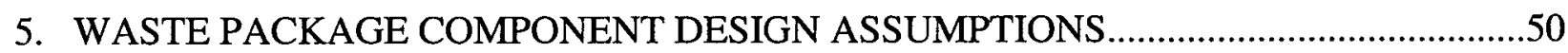

5.1 SOURCE TERM GENERATION ASSUMPTIONS …………................................ 50

5.1.1 Commercial Spent Nuclear Fuel Waste Forms ................................................51

5.1.2 Non-Commercial Spent Nuclear Fuel Waste Forms ..........................................52

5.2 STRUCTURAL ANALYSIS ASSUMPTIONS ………………………....................... 53

5.2.1 Contact Stiffness Between Waste Package and Impact Surface ........................53

5.2.2 Emplacement Pallet Lift ......................................................................................53

5.2.3 Geometry of Collapsed Drift .............................................................................53

5.2.4 First Failure of Inner Vessel Lid for Pressurization Event Sequence ................53

5.2.5 Strain Rate Effect on Material Properties..........................................................54

5.2.6 Uniform Strains .....................................................................................5

5.2.7 Assumptions for Missile Impact Analysis.........................................................55

5.2.8 General Assumptions for Waste Package Drop Analyses.................................55

5.2.9 Assumptions for the Representation of Waste Package Loaded Internals ........59

5.2.10 Assumed Geometry of Lid Lifting Features for Waste Package Tip-Over.......60

5.2.11 Assumed Angular Velocity for Waste Package Swing Down Analysis ...........60

5.2.12 5 DHLW/DOE-Short Waste Package Swing Down Height...............................60

5.2.13 Assumed Geometrical Arrangement of Waste Package for Corner Drop Analysis 


\section{CONTENTS (Continued)}

Page

5.2.14 Additional Assumptions for Waste Package Vertical Drop Analysis ................60

5.2.15 Additional Assumptions for Waste Package Puncture Drop Analysis...............61

5.2.16 Assumptions for Rockfall Upon Waste Package and Drip Shield .....................62

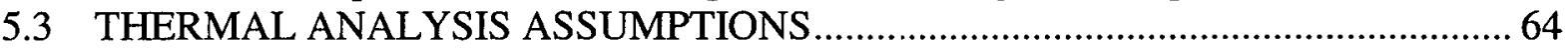

5.3.1 Assumptions for Repository-Scale Thermal Analyses........................................64

5.3.2 Assumptions for Drift-Scale Thermal Analysis ..................................................67

5.3.3 Assumptions for Waste Package-Scale Thermal Analysis.................................68

5.3.4 Assumptions for Fuel Assembly-Scale Thermal Analysis.................................68

5.3.5 Assumptions for Waste Package Fire Analyses ..................................................70

5.3.6 Assumptions for Waste Package/Surface Facility Analyses..............................70

5.3.7 Assumptions for DRIFTFLOW Calculations................................................70

5.3.8 Assumptions for FLUENT Calculations ……...............................................70

5.4 SHIELDING ANALYSIS ASSUMPTIONS............................................................ 71

5.4.1 Use of an Axial Peaking Factor........................................................................71

5.4.2 Homogenization of the Radiation Source Region for Commercial Spent Nuclear Fuel ...............................................................................................71

5.4.3 Homogenization of the U.S. Department of Energy Spent Nuclear Fuel Canister..........................................................................................................71

5.4.4 Omission of Waste Package Internals .............................................................72

5.4.5 Use of a Watt Fission Spectrum ...................................................................72

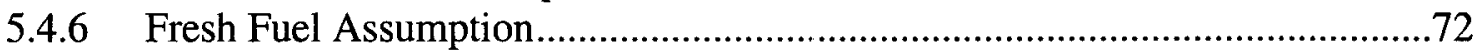

5.4.7 Treatment of Trace Elements ........................................................................72

5.4.8 High-Level Radioactive Waste Glass Source Terms ...........................................72

5.4.9 Infinite Cylinder Representation of a Waste Package in SAS1 Analyses.........73

5.5 CRITICALITY ANALYSIS ASSUMPTIONS …………………............................... 73

6. WASTE PACKAGE COMPONENT ANALYSIS METHODS AND

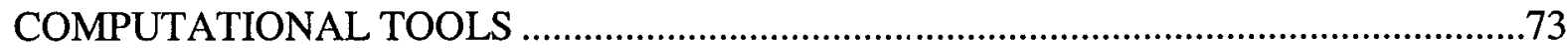

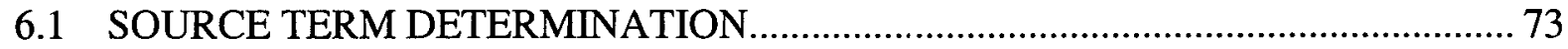

6.1.1 Computational Tools ................................................................................

6.1.2 Description of Pertinent Analyses ...............................................................76

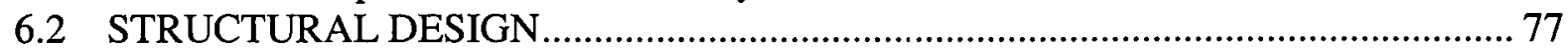

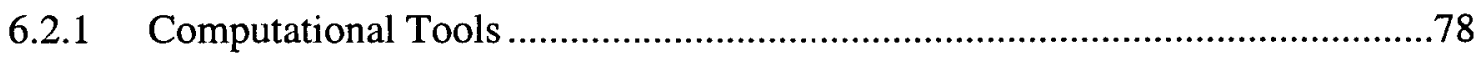

6.2.2 Description of Pertinent Analyses...............................................................78

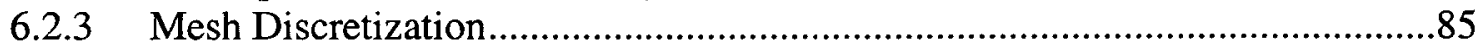

6.2.4 Waste Package Component Design Stress Limits and Failure Criteria .............86

6.2.5 Calculations for True Measures of Ductility .....................................................91

6.2.6 Fracture Mechanics Analyses....................................................................92

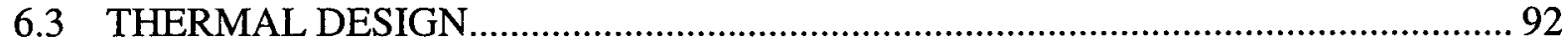

6.3.1 Computational Tools …….......................................................................93

6.3.2 Description of Pertinent Analyses....................................................................94

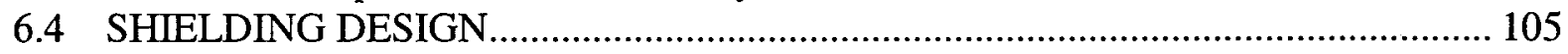

6.4.1 Computational Methods and Tools ……….............................................105

6.4.2 Description of Pertinent Analyses ...................................................................108 


\section{CONTENTS (Continued)}

Page

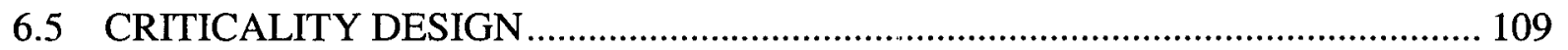

6.5.1 Computational Methods and Tools .................................................................109

6.5.2 Description of Pertinent Analyses ...................................................................109

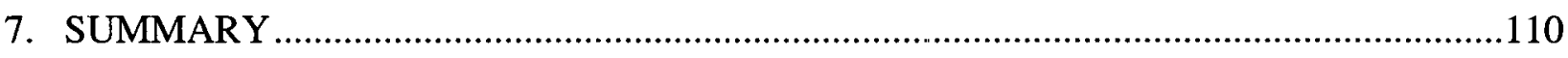

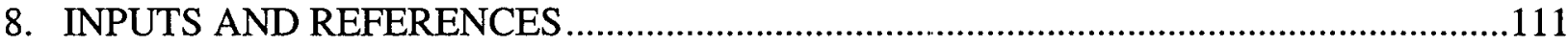

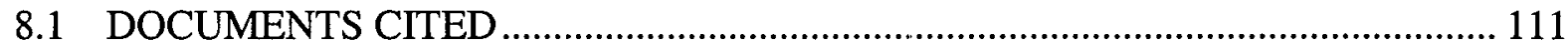

8.2 CODES, STANDARDS, REGULATIONS, AND PROCEDURES ............................ 121

8.3 SOURCE DATA, LISTED BY DATA TRACKING NUMBER ............................... 123 
INTENTIONALLY LEFT BLANK 


\section{FIGURES}

Page

1. Relationship between Methodology Report and other Design Documents ..........................23

2. Waste Packages in a Repository Drift ………................................................................24

3. $\quad$ Underground Layout Configuration ...............................................................................47

4. $\quad$ Flat Plate Representation of Impact Analysis .....................................................................5

5. $\quad$ Adjustments of Host Rock Thermal Transport Properties...................................................65

6. $\quad$ Modes of Heat Transfer In and Near Drift........................................................................67

7. SCALE Representation of the Fuel Pin Cell and Assembly for SAS2H Calculations..........75

8. Calculational Flow for Source Term Generation .................................................................75

9. Key Attributes of Bilinear Stress-Strain Curves .................................................................92

10. Finite Element Representation of Fuel Assembly ..............................................................98

11. Illustration of Two-dimensional Repository Representation .............................................100

12. Illustration of Response Surface Interrogation ……..........................................................101

13. Three-Dimensional Effect Accommodation ……...............................................................102

14. Thermal Analysis Technique Decision Flowchart...............................................................103

15. Multi-scale Thermal Analysis Representation ......................................................................104

\section{TABLES}

1. Sources of Material Compositions Used in Source Term Determinations .............................35

2. Sources of Material Properties for Structural Analyses............................................................39

3. Sources of Material Properties for Thermal Analyses ..........................................................44

4. Initial Temperature Gradient in Host Rock ……………………………………..................66

5. Summary of Thermal Calculations for Waste Package Component Design ........................96 


\section{INTENTIONALLY LEFT BLANK}




\section{EXECUTIVE SUMMARY}

This Executive Summary provides an overview of the methodology being used by the Yucca Mountain Project (YMP) to design waste packages and ancillary components. This summary information is intended for readers with general interest, but also provides technical readers a general framework surrounding a variety of technical details provided in the main body of the report.

The purpose of this report is to document and ensure appropriate design methods are used in the design of waste packages and ancillary components (the drip shields and emplacement pallets). The methodology includes identification of necessary design inputs, justification of design assumptions, and use of appropriate analysis methods and computational tools. This design work is subject to Quality Assurance Requirements and Description. The document is primarily intended for internal use and technical guidance for a variety of design activities. It is recognized that a wide audience including project management, the U.S. Department of Energy (DOE), the U.S. Nuclear Regulatory Commission, and others are interested to various levels of detail in the design methods and therefore covers a wide range of topics at varying levels of detail. Due to the preliminary nature of the design, readers can expect to encounter varied levels of detail in the body of the report. It is expected that technical information used as input to design documents will be verified and taken from the latest versions of reference sources given herein.

This revision of the methodology report has evolved with changes in the waste package, drip shield, and emplacement pallet designs over many years and may be further revised as the design is finalized. Different components and analyses are at different stages of development. Some parts of the report are detailed, while other less detailed parts are likely to undergo further refinement. The design methodology is intended to provide designs that satisfy the safety and operational requirements of the YMP. Four waste package configurations have been selected to illustrate the application of the methodology during the licensing process. These four configurations are the 21-pressurized water reactor absorber plate waste package (21-PWRAP), the 44-boiling water reactor waste package (44-BWR), the 5 defense high-level radioactive waste (HLW) DOE spent nuclear fuel (SNF) codisposal short waste package (5-DHLW/DOE SNF Short), and the naval canistered SNF long waste package (Naval SNF Long). Design work for the other six waste packages will be completed at a later date using the same design methodology. These include the 24-boiling water reactor waste package (24-BWR), the 21pressurized water reactor control rod waste package (21-PWRCR), the 12-pressurized water reactor waste package (12-PWR), the 5 defense HLW DOE SNF codisposal long waste package (5-DHLW/DOE SNF Long), the 2 defense HLW DOE SNF codisposal waste package (2-MCO/2-DHLW), and the naval canistered SNF short waste package (Naval SNF Short).

This report is only part of the complete design description. Other reports related to the design include the design reports, the waste package system description documents, manufacturing specifications, and numerous documents for the many detailed calculations. The relationships between this report and other design documents are shown in Figure 1. 


\section{Overview of the Preliminary Waste Package Component Designs}

Figure 2 shows a typical emplacement drift in the repository with waste packages beneath drip shields sitting on emplacement pallets, which rest on the steel invert structure. The invert structure will be filled with crushed tuff (ballast is not shown in Figure 2). This report applies to ten specific waste package configurations and the ancillary components (i.e., the drip shields and emplacement pallets). Different waste package configurations are designed for different waste forms and have different diameters, lengths, and internal structural arrangements. The internal structures not only separate waste assemblies but also provide thermal shunts for heat removal and neutron absorbers for criticality control. The waste packages are supported by emplacement pallets and protected from dripping water and rockfall in the post-closure period by drip shields.

The fundamental design of the waste packages is a dual cylinder comprised of an inner vessel and an outer corrosion barrier. The bottom end of the waste package has two lids, one for the inner vessel and one for the outer corrosion barrier. The top end of the waste package has three lids, one for the inner vessel and two for the outer corrosion barrier. Removable trunnion collars (not shown in Figure 2) and sleeves at the ends of the waste package provide an interface for lifting and handling operations.

\section{Design Methodology}

Design methodology can be viewed simply as (1) gathering all the design input information; (2) making reasonable assumptions; and (3) selecting analyses methods and computational tools, and (4) defining how design criteria are satisfied. Each of these parts of design methodology is quite extensive and discussed in subsequent detail in this report. The results of specific calculations and analyses performed using the design methodology are reported in the specific calculations.

\section{Design Inputs}

The inputs to waste package component design include design requirements, technical information, and project interfaces. Reference sources for design inputs are documented in the body of this report.

Requirements-Top level requirements originate from the DOE and are presented in Project Requirements Document. Regulations such as 10 CFR Part 63 [DIRS 156605] are included in project level requirements. Design requirements for the geologic repository flow down to design requirements in system description documents for waste package components.

Criteria-The numeric values and other measures whereby satisfaction of the design requirements is demonstrated. The criteria are presented in the Project Design Criteria Document.

Technical Information-Technical information includes physical dimensions, material properties, and radionuclide content. Differences in the physical forms, irradiation histories, and measured radionuclide content, among the commercial SNF, DOE SNF, and HLW are used to generate source terms for pressurized water reactor (PWR) and boiling water reactor (BWR) 
commercial SNF, DOE SNF, and defense HLW. Structural and thermal calculations use standard mechanical and thermal properties.

Interfaces-Waste package component designs interface with other parts of the YMP, include ties to fabrication and handling facilities, preclosure safety analysis, and performance assessment. Some parts of the facilities design are in an early stage at the present time and will not be complete at the time of the License Application submittal. Additional design analysis will be performed, if necessary, as the facilities design matures and risks are evaluated.

\section{Assumptions}

Design Assumptions-These may be divided into two categories. The first category consists of generic assumptions that can be part of a calculation process or computational tool. The second category of assumptions is specific assumptions related to a particular analysis or calculation. The generic assumptions are detailed in the body of this report as well as some specific assumptions where more detail is needed for technical clarification of particular design issues.

Source Term Assumptions-Commercial SNF source terms are approximated by calculating source terms for generic PWR and BWR assemblies at incremental enrichments and burnups and a generic burnup history (without the modeling of outages, intermittent down times, etc.) for fuel depletion calculations. The Babcock \& Wilcox (B\&W) Mark B $15 \times 15$ PWR fuel assembly is selected as the generic PWR lattice for analysis. This lattice has a high initial heavy metal loading and large amounts of stainless steel and Inconel assembly hardware, maximizing fission product generation and activation of structural hardware. For BWR commercial SNF, a General Electric (GE) $8 \times 8 \mathrm{BWR}$ fuel assembly is used. This design has a high initial heavy metal loading and an adequate amount of fuel assembly hardware data. For PWR and BWR fuel, conservatively high fuel mass is added as increased length rather than to increase density. An average uniform specific power over the entire length of the assembly is assumed, and the total irradiation interval is determined as the ratio of the assembly burnup to the specific power. It is assumed that the source terms can be generated for an array of various enrichments, burnups, and decay times and that interpolation can then be used to obtain the source terms of any specific assemblies in the waste stream without requiring explicit modeling of the assemblies. DOE SNF source terms are based on radionuclide inventories for several representative fuel types in the DOE SNF waste stream. For the defense HLW, historical information regarding the inventory at the various sites is used in decay calculations to generate initial radionuclide inventories.

Structural Analysis Assumptions-For a few materials, structural properties are not available but properties for materials with very similar compositions are available and are used in their place. Structural calculations are based on uniform strain and neglect strain rate effects. During handling and lifting, accelerations are assumed to equal $1.0 \mathrm{~m} / \mathrm{s}^{2}$ (total upward acceleration of $10.81 \mathrm{~m} / \mathrm{s}^{2}$ including gravity). Commonly used assumptions are made for friction coefficients. Geometric assumptions are required for missile impact and drop calculations and to evaluate a collapsed drift. Crack evaluations assume single crack propagation. Burst evaluations assume the inner lid fails first.

Thermal Analysis Assumptions-Thermal evaluations used in waste package component analysis use several geometric scales and assumptions appropriate to the scale of the analysis 
made. At the largest scale, pillar modeling used for a three-dimensional analysis assumes an average drift segment. Water movement is ignored in the conduction only model with a specific heat adjustment to account for latent heat of water as rock temperatures pass through the boiling point. At the waste package scale, a two-dimensional analysis is performed at the mid-plane using the highest heat load peaking factor and ignoring axial conduction. An effective conductivity is used for the fuel assemblies.

Shielding and Criticality Analysis Assumptions-The composition of fresh fuel is used to represent the attenuation properties of spent fuel in shielding calculations. Because the radiation source terms assume burnup is uniformly distributed within a SNF assembly, an axial peaking factor is used for shielding analysis near the active fuel region. For three-dimensional shielding calculations, the contents and radiation sources of each waste type are uniformly homogenized with the internal structural components. Criticality methods make conservative assumptions concerning burnup, moderator properties, and poison distributions.

\section{Analysis Methods and Computational Tools}

Numerous calculations are performed to assure the chosen design(s) perform as expected. These calculations are made using several computer software packages specifically suited to different purposes.

Source Term Calculations-Source term calculations provide heat generation rates, photon and neutron spectra and intensities, and radionuclide inventories of commercial SNF assemblies, DOE SNF, and DOE HLW. The heat generation rates are used in thermal evaluations of the waste packages and the host rock of the repository. The photon and neutron sources are used to determine the radiation level surrounding a waste package. The radionuclide inventories are used to determine dose rates due to the release of radionuclides from the waste packages.

Structural Calculations-Structural calculations demonstrate that the waste package, drip shield, and emplacement pallet meet the requirements for normal operations and Category 1 and Category 2 event sequences. Structural calculations fall into the following broad groups:

- Geometric Design

- Normal Operations

- Internal Pressurization

- Impacts on Waste Package or Drip Shield

- Dynamic Impacts on the Waste Package

- Seismic Evaluations

- Residual Stress Reduction.

Waste packages, drip shields, and emplacement pallets can be represented as two-dimensional or three-dimensional finite-element geometries, depending on the symmetry of the design or the loading.

Thermal Calculations-Thermal calculations are performed to assure that fuel cladding, waste package, and rock temperatures do not exceed maximum limits. Thermal analyses include calculations of all three modes of heat transfer: conduction, convection, and radiation. Thermal 
calculations are performed in one, two, or three dimensions; and at geometric scales ranging from individual assembly cross sections to pillar representations of a drift segment. ANSYS is typically used to calculate conduction and radiation heat transfer.

Shielding Calculations-For all waste package designs, radiation dose rates in the axial and radial directions are determined on segments of the waste package surfaces. The results of the shielding calculations allow an estimation of the average operation time of welding equipment, radiolysis-induced corrosion, and the radiation environment outside the waste packages for personnel access.

Criticality Analyses-The methodology to be used for criticality analysis for the repository at Yucca Mountain, Nevada, is detailed for the preclosure period in Preclosure Criticality Analysis Process Report and for the postclosure period in Disposal Criticality Analysis Methodology Topical Report. These reports provide the design requirements; applicable regulations, codes, and standards; summary descriptions of the types of computational tools to be used; and the types of analyses to be performed.

The details of the design methodology in the body of this report are organized around Design Inputs (Section 4), Design Assumptions (Section 5), and Analysis Methods (Section 6). Within each of these, subsections are included for source term, structural, thermal, shielding, and criticality design. The methodology should be considered together with information contained in other design reports and calculation report(s) for specific design issues. 
INTENTIONALLY LEFT BLANK 


\section{ACRONYMS}

ASM

ASME

$\mathrm{B} \& \mathrm{~W}$

BWR

DIRS

DOE

GE

HLW

NRC

NUREG

PWR

SNF

TSPA

YMP
American Society for Metals

American Society of Mechanical Engineers

Babcock \& Wilcox

boiling water reactor

Document Input Reference System

U.S. Department of Energy

General Electric

high-level radioactive waste

U.S. Nuclear Regulatory Commission

NRC Regulation Report

pressurized water reactor

spent nuclear fuel

total system performance assessment

Yucca Mountain Project 


\section{INTENTIONALLY LEFT BLANK}




\section{OBJECTIVE ANI) SCOPE}

This report describes the design inputs, assumptions, analytical methods, and computational tools used to establish the appropriateness of the design of waste package components. Design methodology has progressed over many years as the Yucca Mountain Project (YMP) has evolved and will be further refined as the project matures.

The purpose of this report is to document and ensure appropriate design methods are used in the design of waste packages and ancillary components (the drip shields and emplacement pallets). The methodology includes identification of necessary design inputs, justification of design assumptions, and use of appropriate analysis methods and computational tools. The document is primarily intended for internal use as technical guidance for a variety of design activities. It is recognized that a wide audience including project management, the U.S. Department of Energy (DOE), the U.S. Nuclear Regulatory Commission (NRC), and others are interested to various levels of detail in the design methods. It is expected that technical information used as input to design documents will be verified and taken from the latest versions of reference sources given herein.

The design methodology is intended to provide designs that satisfy the safety and operational requirements of the YMP. Four waste package configurations have been selected to illustrate the application of the methodology during the licensing process. These four configurations are the 21-pressurized water reactor absorber plate waste package (21-PWR AP), the 44-boiling water reactor waste package (44-BWR), the 5 defense high-level radioactive waste (HLW) DOE spent nuclear fuel (SNF) codisposal short waste package (5-DHLW/DOE SNF Short), and the naval canistered SNF long waste package (Naval SNF Long). The compliance of these waste packages to requirements delineating the safety strategy is documented in three reports:

- Commercial SNF Waste Package Design Report (BSC 2004 [DIRS 168217])

- HLW/DOE SNF Codisposal Waste Package Design Report (BSC 2004 [DIRS 166895])

- Naval Waste Package Design Report (BSC 2004 DIRS 166894)

Design work for the other six waste packages will be completed at a later date using the same design methodology. These include the 24-boiling water reactor waste package (24-BWR), the 21-pressurized water reactor control rod waste package (21-PWRCR), the 12-pressurized water reactor waste package (12-PWR), the 5 defense HLW DOE SNF codisposal long waste package (5-DHLW/DOE SNF Long), the 2 defense HLW DOE SNF codisposal waste package (2-MCO/2-DHLW), and the naval canistered SNF short waste package (Naval SNF Short).

This revision of the methodology report has evolved with changes in the waste package, drip shield, and emplacement pallet designs over many years and will be further revised as the design is finalized. Different components and analyses are at different stages of development. Some parts of the report are detailed, while other less detailed parts are likely to undergo further refinement. 
This report is only part of the complete design description. Other reports related to the design include the design reports, waste package system description documents, manufacturing specifications, and numerous documents for the many detailed calculations. The relationships between the Methodology Report and other design documents are shown in Figure 1.

Figure 2 shows a typical emplacement drift in the repository with waste packages beneath drip shields sitting on emplacement pallets, which rest on the steel invert structure. The invert structure will be filled with crushed tuff (the ballast is not shown in Figure 2). This report applies to ten specific waste package configurations and the ancillary components (i.e., the drip shields and emplacement pallets). Different waste package configurations accommodate different waste forms and have different diameters, lengths, and internal structural arrangements. The internal structures not only separate waste assemblies but may also provide thermal shunts for heat removal and neutron absorbers for criticality control. The waste packages are supported by emplacement pallets and protected from post-closure rnoisture and rockfall by drip shields. 


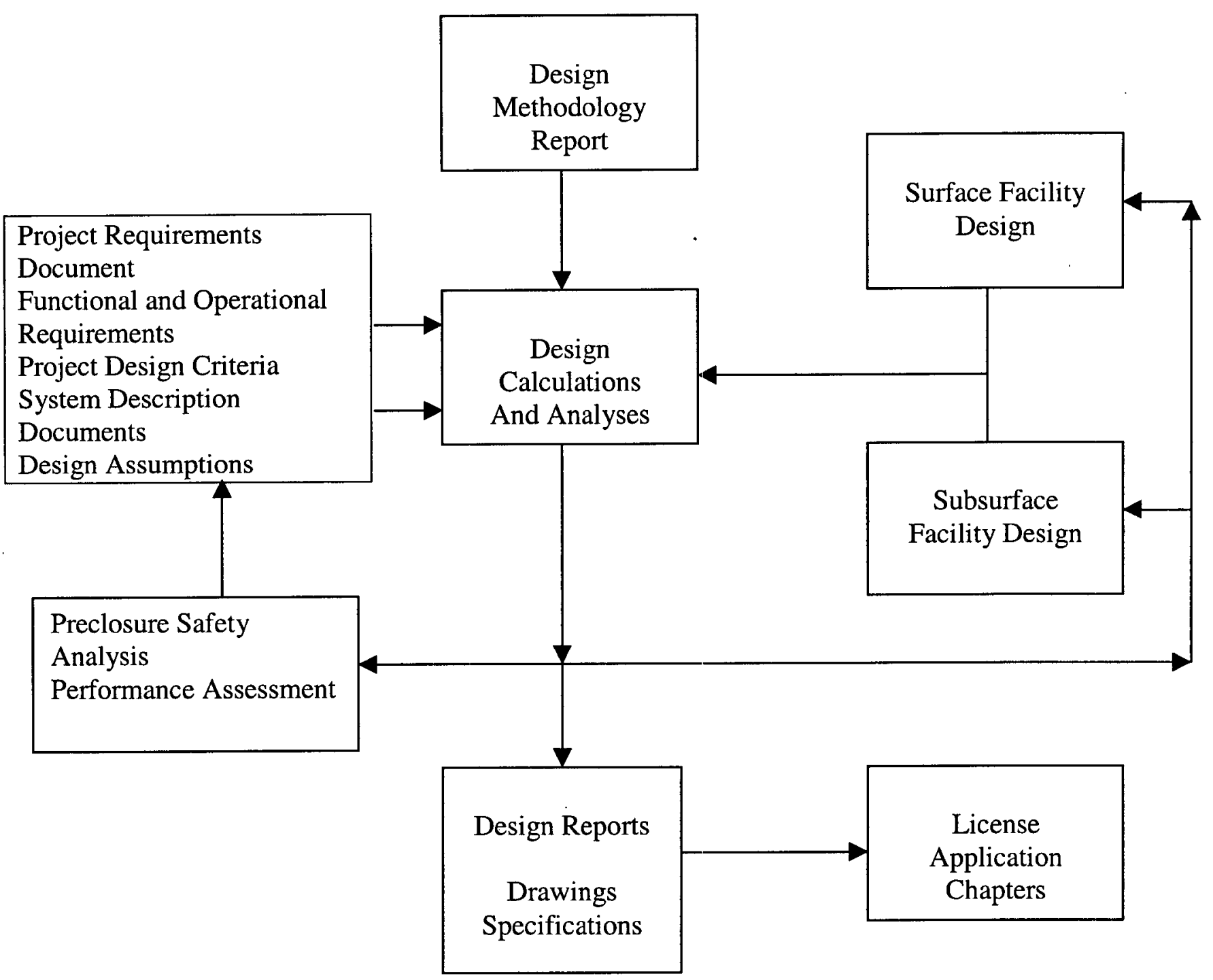

Figure 1. Relationship between Methodology Report and other Design Documents 


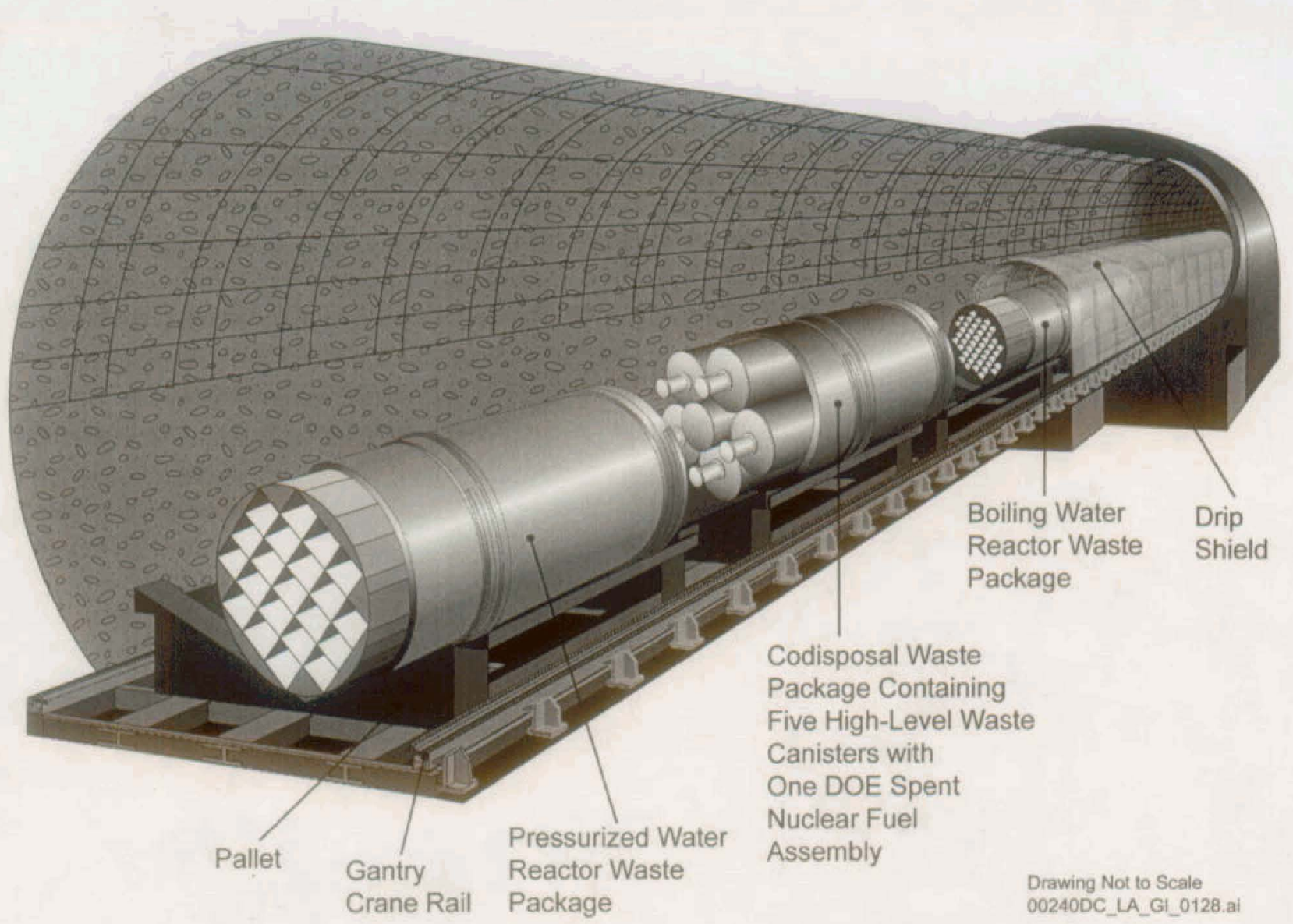

Figure 2. Waste Packages in a Repository Drift

The fundamental design of the waste packages is a dual cylinder comprised of an inner vessel and an outer corrosion barrier. The bottom end of the waste package has two lids, one for the inner vessel and one for the outer corrosion barrier. The top end of the waste package has three lids, one for the inner vessel and two for the outer corrosion barrier. Removable trunnion collars (not shown in Figure 2) and sleeves at the ends of the waste package provide an interface for lifting and handling operations.

Design methodology can be viewed simply as (1) gathering all the design input information; (2) making reasonable assumptions; (3) selecting analyses methods and computational tools; and (4) defining how design criteria are satisfied. Each of these parts of design methodology is quite extensive and discussed in subsequent detail in this report. The results of specific calculations and analyses performed using the design methodology are reported in the specific calculations.

The scope of this report includes computational methods for determining the source term, structural performance, thermal conditions, and shielding protection. The design inputs, assumptions, methods, and tools have been chosen to obtain designs that ensure defense-in-depth as well as satisfy requirements on system performance. Such requirements include those imposed by federal regulations from the DOE and the NRC, and those imposed by the YMP to meet repository performance goals. 
The design methods and techniques described in this report are to be used in design activities producing input to the License Application of all waste package components. These include all waste package configurations, the emplacement pallets, and drip shields.

Waste package components are evaluated for the preclosure and postclosure periods. During preclosure the waste packages do not breach, do prevent criticality, and do maintain an environment for physical and chemical stability of the waste. During postclosure the waste packages restrict the transport of radionuclides to the outside of the waste package, greatly decrease the likelihood of criticality, and delay the degradation of the waste form. Various structural, thermal, and criticality analyses are performed to assure performance for normal operations and Category 1 and Category 2 sequence events. These analyses provide confidence that the waste packages will survive hazards such as drops, rockfall, seismic motion, and fires without breaching during preclosure.

\section{QUALITY ASSURANCE}

This document is subject to Quality Assurance Requirements and Description (DOE 2004 [DIRS 168669]) requirements. This document was developed in accordance with AP-3.11Q, Technical Reports.

\section{COMPUTER SOFTWARE AND MODEL USAGE}

No computer software or models were used in the generation of this report; however, computer software that is used to implement the methodology presented in this report is described in detail in Section 6.

Design methods use several computer software packages, including:

SCALE V.4.4.A (HP-UX B 10.20 operating systern, Software Tracking Number 101294.4A-00), for criticality and shielding (CRWMS M\&O 2000 [DIRS 154394])

- $\quad$ SAS2 module, for depletion analysis

- $\quad$ ORIGEN-S module, for decay calculations

- MCNP V.4B2LV (HP-UX B 10.20 operating system, Software Tracking Number 30033 V4B2LV), for neutron/photon/electron transport for shielding calculations (CRWMS M\&O 1998 [DIRS 154060])

- ANSYS V.5.6.2 (HP-UX 11.0 operating system, Software Tracking Number 103645.6.2-01), or many structural and thermal calculations (BSC 2002 [DIRS 159357])

- LS-DYNA V.960.1106 (HP-UX 11.0 operating system, Software Tracking Number 10300-960.1106-00.), for dynamic structural calculations (BSC 2002 [DIRS 158898])

MATHCAD, for solving systems of equations (MathSoft 1998 [DIRS 119437]) 
- FLUENT V.6.0.12 (HP-UX 11.00 operating system, Software Tracking Number 105506.0.12-00), for some convective flow and heat transfer calculations (Fluent, Inc. 2003 [DIRS 163001])

- DRIFTFLOW V1.0 (PC Windows 2000 operating system, Software Tracking Number 10722-1.0-00), for ventilation calculations (BSC 2002 [DIRS 163090]).

Software used for criticality analysis is described in the Preclosure Criticality Analysis Process Report (BSC 2004 [DIRS 169590]) and in the following model reports: Errata for Isotopic Model Report for Commercial SNF Burnup Credit (BSC 2003 [DIRS 168037]), Configuration Generator Model for In-Package Criticality (BSC 2003 [DIRS 165629]), Geochemistry Model Validation Report: External Accumulation Model (BSC 2001 [DIRS 156324]), Geochemistry Model Validation Report: Material Degradation and Release Model (BSC 2001 [DIRS 156790]), and the Criticality Model Report (BSC 2003 [DIRS 165733]).

The computer codes listed above (except MATHCAD) are maintained under YMP Software Configuration Management to assure quality control of the software used in performing design calculations. These are the latest, but not only, versions of the software packages under Software Configuration Management. Some calculations use earlier versions and other operating systems, and individual calculations document the actual version used for specific applications. In the remainder of this document, the software is referred to by name only, without specific version numbers and operating systems, but the controlled code versions are the only code versions to be used.

\section{WASTE PACKAGE COMPONENT DESIGN INPUTS}

The first levels of input to waste package component design are requirements established through a process starting with the Nuclear Waste Policy Amendments Act of 1987 [DIRS 100016]. These requirements are further developed into design requirements and assigned to structures, systems, and components through system description documents. Compliance with the requirements is demonstrated by satisfaction of design criteria. In order to develop the necessary design output, additional information and parameters are required, including radionuclide parameters and structural and thermal properties of materials. Finally, inputs are developed through interfaces with equipment and processes assigned to other organizations. The next three subsections will describe the source of requirements, information and parameters, and interface inputs.

\subsection{REQUIREMENTS}

Structures, systems, and components of the repository are designed to specified requirements. Requirements for the repository originate from the DOE and are presented in Project Requirements Document (Canori and Leitner [DIRS 166275]). Laws and statutes, such as 10 CFR Part 63 [DIRS 156605], are included in these project level requirements. The Project Requirements Document decomposes these requirements to the point that each can be assigned as appropriate. The assigned organization uses the requirement or further breaks it down by deriving daughter requirements. Project Functional and Operational Requirements (Siddoway 2003 [DIRS 163904]) generates requirements based on functions of the repository. All of these 
requirements are ultimately assigned to a system and are published in a System Description Document. Some of these requirements will point to criteria in the Project Design Criteria Document (Minwalla 2003 [DIRS 161362]). Criteria are the numerical values and other measures whereby satisfaction of the design requirements is demonstrated. Identifying criteria in a system description document assigns the criteria to structures, systems, and components of the system description document. Design criteria consist of the codes, standards, general discipline design criteria, and load combinations that are specified for use on the project.

\subsubsection{Assigned Requirements}

The Analysis and Component Design organization is concerned with requirements in several system description documents. The Analysis and Component Design organization is directly responsible for three system description documents that deal with waste packages and waste package closure:

- DOE and Commercial Waste Package System Description Document (BSC 2004 [DIRS 167273])

- Naval Spent Nuclear Fuel Waste Package System Description Document (BSC 2004 [DIRS 167272])

- Waste Package Closure System Description Document (BSC 2004 [DIRS 167278]).

The Analysis and Component Design organization is also responsible for the requirements and criteria for waste package drip shields and emplacement pallets, which are described in the Subsurface Facility Description Document (BSC 2004 [DIRS 170265]).

\subsubsection{Design Philosophy}

Requirements drive the design of the waste package, drip shield, and waste package pallet. There are two primary conditions or periods of concern. These are the preclosure period and the postclosure period. For preclosure the waste package is relied on to contain the waste form within its boundary, prevent criticality, and preserve the physical and chemical stability of the waste form. For postclosure the waste package is relied on to restrict the transport of radionuclides to the outside of the waste package, greatly decrease the likelihood of criticality, and delay the degradation of the waste form. Structurally, waste packages consist of an American Society of Mechanical Engineers (ASME 2001 [DIRS 158115], Section III) inner vessel of 316 stainless steel and an outer corrosion barrier made of Alloy 22 N06022. In order to show that the waste package will perform these functions adequately, many analyses must be performed on them. The design philosophy requires the waste package to survive external hazards such as drops, rockfall, seismic motion, and fires without breaching. It must also show that the waste form stays within specified thermal limits throughout its life. A calculation will be done in accordance with the ASME Boiler and Pressure Vessel Code (ASME 2001 [DIRS 158115]) to account for the pressure retention by the inner vessel. Additional calculations will be done outside the code for the conditions identified for off-normal event sequences. 


\subsubsection{Codes and Standards}

Some codes and standards are imposed through the Project Design Criteria Document (Minwalla 2003 [DIRS 161362]), while the design engineer selects others. Codes and standards include industry codes such as the ASME Boiler and Pressure Vessel Code (ASME 2001 [DIRS 158115]) and standards such as those published by American National Standards Institute. Publications by the NRC are included, which consist of:

- Regulatory Guides-Rules for specific areas of concern that if followed are acceptable ways to meet NRC requirements

- NRC Regulation Reports (NUREGs)—Guidance documents from the NRC

- NUREG/Contractor Reports (NUREG/CRs)—Information published by the NRC.

The Analysis and Component Design organization uses these selected codes and standards as follows:

- Commitments to the regulators-credit is taken for meeting the item. Examples include ASME Boiler and Pressure Vessel Code, Section III, Division 1 (ASME 2001 [DIRS 158115] and ANSI N14.6-1993 [DIRS 102016]).

- Accepted Source of Methods or Data-the method or data is used. For example, NUREG/CR-6608 (Witte et al. 1998 [DIRS 136115]) addresses the impact of large packages during a drop event.

- Internal Guidance to Analysis and Component Design - the item was reviewed and used for design input. For example, NUREG-1804 (NRC 2003 [DIRS 163274]) is used to ensure that items identified by the NRC are addressed.

\subsubsection{Commitment Codes and Standards}

- ASME Boiler and Pressure Vessel Code (ASME 2001 [DIRS 158115], Section III, Division 1) is used for general structural analysis and fabrication of the inner vessel

- ANSI N14.6-1993 [DIRS 102016] addresses lifting devices for large packages

- ANSI/ANS-6.1.1-1977 [DIRS 107016] presents flux-to-dose rate conversion factors for neutron and gamma radiation.

\subsubsection{Accepted Source or Guidance}

Structural:

- ASTM A 887-89 (Reapproved 2000) [DIRS 154062], Standard Specification for Borated Stainless Steel Plate, Sheet, and Strip for Nuclear Application 
- NUREG/CR-1815 (Holman and Langland 1981 [DIRS 145192]) addresses the fracture toughness requirements of cylindrical shells for packages

- NUREG/CR-0481 (Rack and Knorovsky 1978 [DIRS 145185]) addresses elastic plastic data for structural analyses

- NUREG/CR-6608 (Witte et al. 1998 [DIRS 136115]) addresses the impact of large packages during a drop event

- NUREG/CR-6322 (Lee and Bumpas 1995 [DIRS 154068]) provides a method for evaluating the buckling stresses in SNF baskets

- NUREG/CR-4554 (Gerhard et al. 1992 [DIRS 145318]) presents information regarding the structural stresses for large packages during a drop event

- NUREG-0612 (NRC 1980 [DIRS 104939]) describes the requirements for lifting and handling large packages such as waste packages

- Dynamic Impact Effects on Spent Fuel Assemblies (Chun et al. 1987 [DIRS 144357]) describes the effects on spent fuel of high g-loads from impact.

Thermal:

- "Level 2 Directed Baseline Change" (Arthur 2003 [DIRS 164983]) specifies that the clad temperature limit is $350^{\circ} \mathrm{C}$

- ASME Boiler and Pressure Vessel Code (ASME 2001 [DIRS 158115], Section III, Division 1, Subsection NB-1120) sets temperature limits for structural alloys.

Source Term and Shielding:

- NUREG/CR-5625 (Hermann et al. 1994 [DIRS 154045]) provides technical support for the NRC decay heat guide using the SAS2H and ORIGEN-S analysis sequence of the SCALE system

- NUREG-1536 (NRC 1997 [DIRS 101903]) provide guidance for evaluating shielding of SNF packages

- NUREG-1617 (NRC 2000 [DIRS 154000]) provide guidance for evaluating shielding of SNF packages

- NUREG-1567 (NRC 2000 [DIRS 149756]) provide guidance for evaluating shielding of SNF packages. 


\subsubsection{Use of Codes and Standards}

\subsubsection{Structural Codes and Standards}

The ASME Boiler and Pressure Vessel Code (ASME 2001 [DIRS 158115]) is used for structural analysis in general and fabrication of the inner vessel. This is consistent with the NRC requirements to use codes and standards based on accepted industry technology (NRC 2003 [DIRS 163274]).

The thickness of the Yucca Mountain waste package inner vessel is determined by the postulated accident loads. Therefore it will satisfy the ASME Code Design Loads, which are based on pressure and loaded dead weight. The inner vessel will be constructed in accordance with all provisions of the ASME Section III, Division 1, Class 2 Code requirements including application of the ASME N Code Symbol Stamp. The ASME design work will be performed by an ASME Designer of Record, not by Bechtel SAIC Company, LLC.

Alloy 22 N06022 is specifically included as a corrosion barrier and is not a pressure vessel. It will however be constructed in accordance with specific applicable Section III technical requirements including the material, fabrication, and examination requirements and in accordance with selected administrative requirements of the ASME Code. The postulated dynamic loads will be evaluated against appropriate criteria. The outer corrosion barrier will be fabricated in accordance with the applicable specified provisions of NC-2000 (Materials), NC4000 (Welding), and NC-5000 (Nondestructive Examination).

The waste form is placed within the inner vessel, which is partitioned by divider plates to separate waste in canisters or partitioned by basket assemblies for individual elements. Divider plates and basket assemblies facilitate loading and aid in transferring heat from the fuel to the outside. The internal basket assemblies within the inner vessel also ensure proper geometry of the fuel and provide additional neutron poisons where required in order to preclude criticality events. The ASME Code does not address criticality issues, and therefore the ASME Code rules do not apply to the vessel internals. The internal basket assemblies will be attached to the interior of the inner vessel. These attachment welds will be performed in accordance with ASME requirements (ASME 2001 [158115], Section III, Division 1, Class 2).

The waste package is lifted in the vertical position for loading and rotated into the horizontal position for emplacement by use of trunnion collars. The trunnion collar is a removable lifting device attached to the corrosion resistant barrier. The ASME Code is not applicable to the trunnion collar. The function of the trunnion collar is to lift the waste package. Materials will be selected to ensure that there will be no galling of the corrosion barrier material. The design and fabrication requirements will be in accordance with ANSI N14.6-1993 [DIRS 102016].

The ASME Code (ASME 2001 [DIRS 158115], Section III, Division I, Subsection NC) was selected for the code-compliant design and fabrication of the waste packages. The implementation of the ASME Code is described in BSC Position on the Use of the ASME Boiler and Pressure Vessel Code for the Yucca Mountain Waste Packages (BSC 2003 [DIRS 165058]). For the code design, the only part of the waste package considered to be a pressure vessel is the stainless steel type 316 inner vessel. For all other components of the waste package, the ASME 
code is used as guidance, either through the use of conservative material properties or conservative stress limits. For credible preclosure event sequences and the assessment of those event sequences, the code and supporting code interpretations are used to formulate layered defensible material failure criteria. While the seal welds are anticipated to be sound welds, no credit for resistance against dynamic events is taken, as these are partial-penetration welds. Therefore, for dynamic structural events where the inner vessel in the vicinity of the seal welds may be reasonably anticipated to experience considerable loads, these welds are not credited to maintain the hermeticity of the inner vessel. To maintain containment of the waste form, it must be shown that the outer corrosion barrier does not breach.

The following are specific applications of structural standards used in design.

ASTM A 887-89 (Reapproved 2000) ([DIRS 154062], Table 1), Standard Specification for Borated Stainless Steel Plate, Sheet, and Strip for Nuclear Application, prescribes a range of boron-10 contents that are approved for the specification as well as two metallurgical processes, which may be used to manufacture the borated stainless steel. Plate, sheet, and strip are the approved borated stainless steel forms because the manufacturing process assures an even distribution of boron in the resulting steel. Local increases in boron content are undesirable because boron acts as a hardener of stainless steel and reduces the ductility of the alloy. Extrusions and castings would require different metal production processes, and control of the distribution of the boron in the stainless steel alloy would have to be assured. Boron contents in ranges from 0.20-0.29 (Type 304B) up to 1.75-2.25 percent (Type 304B7) are approved by the specification (ASTM A 887-89 (Reapproved 2000) [DIRS 154062]).

The two metallurgical processes used to produce borated stainless steel are a traditional metal melt technology (Grade B) and a more modern powder metallurgy process (Grade A). The powder metallurgy process results in a more even distribution of boron in the alloy, and the ductility is improved compared to the wrought (metal melt) process. Because the borated stainless steel is not a load-carrying structural component in existing waste package SNF basket designs, the Grade B alloy is acceptable.

ASME Code Case N-510-1 (ASME 2001 [DIRS 158115]) specifies the code material properties for evaluating 304 stainless steel with boron to allow structural credit to be taken for this material under construction of Section III, Subsections NF and NG components. This code case describes the mathematical combination of principal stresses in the stainless steel to determine the effective stress that could cause failure of the structure, and it also provides the limits and limitations on the use of these materials.

ASME Code Case N-284-1 (ASME 2001 [DIRS 158115]) specifies a methodology for evaluating buckling stresses in a cylindrical body. This code case describes the mathematical combination of principal stresses in a cylindrical shell to determine the effective stress that could cause buckling collapse of the cylinder. Code Case N-284 provides factors of safety that are used in the calculation, but it should be noted that these factors only apply when the ASME Code Case N-284 methodology is used. It is inappropriate to use these factors of safety if buckling is evaluated using classical crippling and buckling analysis techniques. This is because the Code Case N-284 calculation methodology contains an imbedded measure of conservatism that is not necessarily present in classical methods. 
ANSI N14.6-1993 [DIRS 102016] provides a standard for crane and lifting devices for large packages. This standard provides guidance on seismic requirements for the crane and lift fixtures and is normally applied in conjunction with NUREG-0612 (NRC 1980 [DIRS 104939]); thus, ANSI N14.6-1993 [DIRS 102016] should be used as supplemental information for trunnion design.

NUREG-0612 (NRC 1980 [DIRS 104939]) describes the requirements for lifting and handling of large packages such as the waste package. The standard explicitly applies to heavy loads lifted near or over spent fuel pools at nuclear reactor facilities but is also applied to storage and transport cask lifting devices. Thus, the standard can be applied to the waste package. NUREG-0612 specifies that all components that participate in the load path must be designed to a safety factor of six against yield and a factor of ten against ultimate. The standard implicitly includes a dynamic factor to account for "bounce" when lifting and lowering large objects. The standard permits load splitting between redundant load paths, so that if a redundant lifting yoke is used, a factor of three against yield is used for each separate yoke and a factor of five against ultimate is applied. These factors ensure that structural failure of lifting components will not occur in normal conditions, but it is the responsibility of the facility personnel to ensure that the lifting attachments are securely engaged to the large package. A positive means of verification of lifting fixture engagement is necessary, and in general, it is necessary to provide a flat surface to set the package on. Guide plates to center a waste package in a specific location in a pool or dry location are not recommended as such guide devices have caused disengagement of the lifting fixtures for storage cask systems. The particular form of engagement of the lifting fixtures and the waste package is not specified by the standard; only the structural load path requirements are specified.

NUREG/CR-1815 (Holman and Langland 1981 [DIRS 145192]) provides a methodology to determine the fracture toughness requirements of cylindrical shells for packages with a thickness less than 4 inches. The methodology allows the steel type used in the design of a package to be evaluated to ensure that sufficient ductility exists over the operating temperature range of the package. Cold temperatures reduce the ductility of steel alloys, and a substantial temperature margin is provided through the use of these documents to prevent brittle fracture. Metals that do not comply with the methodology should not be used for structural components of the waste package.

NUREG/CR-0481 (Rack and Knorovsky 1978 [DIRS 145185]) addresses elastic/plastic data for structural analyses and attempts to provide a methodology for evaluating the effects of temperature and strain-rate upon metals such as stainless steel. This approach is useful because strain-rate data are normally measured at room temperature, and the waste package is operated over a range of temperatures from below room temperature to much higher than room temperature. The methodology can be used to evaluate the results provided by finite-element structural analysis tools to ensure that the data used by the analysis system is appropriate. The waste package could experience considerable plastic deformation of the package ends in some drop accident scenarios, and a rockfall could similarly cause substantial deformation of the waste package at any location along its length. These accident scenarios would occur at quite different temperatures, and therefore the structural parameters over the elastic/plastic regime must be evaluated. One effect noted in NUREG/CR-0481 is that the shape of the stress-strain curve is essentially the same at different temperatures, but it was also noted that dynamic strain aging 
might occur at elevated temperatures (over $200^{\circ} \mathrm{C}$ ), even in austenitic stainless steels. Also, a significant variation in material properties was observed in different heats of stainless steel.

NUREG/CR-6608 (Witte et al. 1998 [DIRS 136115]) cliscusses the impact of large packages during a drop event, especially with respect to the modeling of the impact target. NUREG/CR6608 describes acceptable methods of describing a crushable/breakable impact pad such as a reinforced concrete pad, which is more realistic than an unyielding surface but still provides a conservative calculation. Using a crushable impact pad would reduce the g-loads applied to the waste package and SNF basket substantially. The design methodology presently uses an unyielding surface to model the impact target for drop analyses. Future design methodology may consider a crushable/breakable impact pad, which would provide an additional safety margin where desired.

NUREG/CR-6322 (Lee and Bumpas 1995 [DIRS 154068]) provides a methodology for evaluating the buckling stresses in SNF baskets by determining the stability capacity of the basket based on analysis of the individual structural components of the basket. The individual components are treated as columns, beam-columns, and plates. The methodology required for determining buckling stresses and comparing these stresses to the acceptance criteria of the ASME Boiler and Pressure Vessel Code (ASME 2001 [DIRS 158115]) is presented in NUREG/CR-6322. The resulting methodology is acceptable to the NRC for transport casks and is thus applicable to waste packages for handling drop accident scenarios.

NUREG/CR-4554 (Gerhard et al. 1992 [DIRS 145318]) provides information regarding the structural stresses for large packages during a drop event and is applicable to the waste package. It is useful for comparison of stresses calculated for the waste package to those calculated for a commercial cask.

Dynamic Impact Effects on Spent Fuel Assemblies (Chun et al. 1987 [DIRS 144357]), is a de facto standard for the ability of SNF to withstand the effects of high g-loads in an impact, which was developed by Lawrence Livermore National Laboratory for the NRC.

\subsubsection{Thermal Codes and Standards}

Methods of thermal analysis are discussed in various standard review plans. NUREG-1536 (NRC 1997 [DIRS 101903]) and NUREG-1567 (NRC 2000 [DIRS 149756]) discuss storage, and NUREG-1617 (NRC 2000 [DIRS 154000]) discusses transportation.

Other relevant standards include the ASME Code (ASME 2001 [DIRS 158115], Section III, Division 1, Subsection NB, NB-1120), which sets the temperature limit for containment structural alloys, states that the ASME Code is not applicable to materials subjected to temperatures outside a certain range (ASME (2001 [DIRS 158115], Tables 2A, 2B, and 4 of Section II, Part D, Subpart 1), essentially setting the temperature limit for reactor vessels alloys to $800^{\circ} \mathrm{F}$. The $\mathrm{DOE}$ has specified the maximum cladding temperature limit at $350^{\circ} \mathrm{C}$ for commercial Zirconium clad fuel (Arthur 2003 [DIRS 164983], p. 3).

A reference relevant to temporary SNF storage at the repository surface facility is Recommended Temperature Limits for Dry Storage of Spent Light Water Reactor Zircaloy-Clad Fuel Rods in 
Inert Gas (Levy et al. 1987 [DIRS 144349]). This publication provides methods for evaluation of fuel rod pressure at elevated temperatures and provides temperature limits for a desired fortyyear storage period. The ability of irradiated Zircaloy cladding to provide a barrier against the release of radioactive material is evaluated as a function of thermal damage to the cladding. Damage is expressed in terms of diffusion-controlled cavity growth of micro-flaws in the Zircaloy matrix.

\subsubsection{Shielding and Source Term Codes and Standards}

Standards for performing shielding and source term calculations include Code of Federal Regulations, American National Standards Institute standards, and NRC Regulatory Guides and NUREG reports. The nuclear engineering codes and standards applicable to the YMP are listed in the Project Design Criteria Document (Minwalla 2003 [DIRS 161362], Section 4.9.1.1). Shielding and source term methodologies follow the recommendations of appropriate NUREGs to ensure compliance with applicable American National Standards Institute standards and NRC Regulatory Guides.

\subsubsection{Criticality Safety Codes and Standards}

The standards for criticality safety analysis principally are NUREGs and standards published by the American National Standards Institute and the American Nuclear Society. Applicable criticality codes and standards for the YMP are listed in the Project Design Criteria Document (Minwalla 2003 [DIRS 161362], Section 4.9.2.1). The storage and transportation standard review plans (Section 4.1.4.2) also present data regarding criticality safety analyses for packages. Further information is contained in the Disposal Criticality Analysis Methodology Topical Report (YMP 2003 [DIRS 165505]) and the supporting model reports.

\subsection{INFORMATION AND PARAMETERS}

A considerable amount of technical information and parameters is needed as input to waste package, emplacement pallet, and drip shield design. This input is used in source term, structural, thermal, and shielding analyses.

\subsubsection{Source Term}

The decay of radionuclides in SNF and HLW is the source of decay heat and radiation. Source term calculations are performed for scores of decay chains to determine radionuclide concentrations, radiation fields, and decay heat. The inputs to source term calculations are needed for a wide range of waste forms. Differences in the physical forms among commercial SNF, DOE SNF, and DOE HLW lead to very different input requirements for generating the source terms for the representative waste forms. Similarities between PWRs and BWRs result in common input requirements; geometric and operational differences between the two reactor types also create input requirements that are uniquely applicable to each.

\subsubsection{Inputs for Commercial Spent Nuclear Fuel Source Term Determinations}

Sources for material compositions (other than uranium dioxide) used to determine commercial SNF source terms are listed in Table 1. For each material, the maximum permissible amount of 
cobalt is incorporated, except for the stainless steels, where a cobalt impurity of 0.08 weight percent (wt\%) is used (Ludwig and Renier 1989 [DIRS 146398], p. 45). For SS-348H, a cobalt impurity of $0.2 \mathrm{wt} \%$ is used (ASME 2001 [DIRS 158115], Section II, Part A, SA-240, Table 1). The remaining elements are representative of the material compositions for each material, but they are biased towards the maximum amount of tin, nickel, and niobium because these lead to larger gamma sources.

Table 1. Sources of Material Compositions Used in Source Term Determinations

\begin{tabular}{|l|l|}
\hline \multicolumn{1}{|c|}{ Material } & \multicolumn{1}{|c|}{ Source } \\
\hline UNS $^{\text {a }}$ R6802 (Zircaloy-2) & $\begin{array}{l}\text { Standard Specification for Wrought Z'irconium Alloy Seamless Tubes for Nuclear } \\
\text { Reactor Fuel Cladding. Philadelphia, Pennsylvania: American Society for Testing and } \\
\text { Materials (ASTM B 811-90 [DIRS 131753], Table 2) }\end{array}$ \\
\hline UNS R60804 (Zircaloy-4) & $\begin{array}{l}\text { Standard Specification for Wrought Zirconium Alloy Seamless Tubes for Nuclear } \\
\text { Reactor Fuel Cladding. Philadelphia, Pennsylvania: American Society for Testing and } \\
\text { Materials (ASTM B 811-90 [DIRS 131753], Table 2) }\end{array}$ \\
\hline UNS S30400 (SS-304) & $\begin{array}{l}\text { 2001 ASME Boiler and Pressure Vessel Code (includes 2002 addenda) (ASME 2001 } \\
\text { [DIRS 158115], Section IIA, SA-240, Table 1) }\end{array}$ \\
\hline UNS S30403 (SS-304L) & $\begin{array}{l}\text { 2001 ASME Boiler and Pressure Vessel Code (includes 2002 addenda) (ASME 2001 } \\
\text { [DIRS 158115], Section IIA, SA-240, Table 1) }\end{array}$ \\
\hline UNS S30200 (SS-302) & $\begin{array}{l}\text { Standard Specification for Heat-Resisting Chromium and Chromium-Nickel Stainless } \\
\text { Steel Plate, Sheet, and Strip for Pressure Vessels (ASTM A 240/A 240M-97a [DIRS } \\
\text { 102769] Table 1) }\end{array}$ \\
\hline SS-348H & $\begin{array}{l}\text { Standard Specification for Heat-Resisting Chromium and Chromium-Nickel Stainless } \\
\text { Steel Plate, Sheet, and Strip for Pressure Vessels (ASTM A 240/A 240M-97a [DIRS } \\
\text { 102769] Table 1) }\end{array}$ \\
\hline Inconel-718 & Product Handbook (Inco Alloys International 1988 [DIRS 130835], p. 11) \\
\hline Inconel-X-750 & Product Handbook (Inco Alloys International 1988 [DIRS 130835], p. 11) \\
\hline Stainless Steel CF3M & $\begin{array}{l}\text { Properties and Selection: Stainless Steels, Tool Materials and Special-Purpose } \\
\text { Metals. Volume 3 of Metals Handbook (ASM 1980 [DIRS 104317], p. 95) }\end{array}$ \\
\hline
\end{tabular}

NOTE: ${ }^{a}$ UNS = Unified Numbering System.

Elemental impurities in the uranium dioxide of the fuel are given in Standard-and ExtendedBurnup PWR and BWR Reactor Models for the ORIGEN2 Computer Code (Ludwig and Renier 1989 [DIRS 146398], Table 5.4).

The isotopic composition of commercially available uranium is provided by the empirical relationships in Sequoyah Unit 2, Cycle 3 - Volume 2 of Scale-4 Analysis of Pressurized Water Reactor Critical Configurations (Bowman et al. 1995 [DIRS 123796], p. 20).

Neutron flux scaling factors are also required for regions outside the active fuel in order to determine neutron-activated gamma source terms. These scaling factors are 150 percent of those provided by Activation Measurements and Comparison with Calculations for Spent Fuel Assembly Hardware. Volume 1 of Spent Fuel Assembly Hardware: Characterization and 10 CFR 61 Classification for Waste Disposal (Luksic 1989 [DIRS 120506], Table S.1, p. vi).

The presence of corrosion products (crud) on the fuel is also accounted for in the source term determination. This is obtained from: 
- Standard Review Plan for Spent Fuel Dry Storage Facilities (NRC 2000 [DIRS 149756], Table 9.2)

- Spent Fuel Corrosion Product and Fuel Cleaning Assessment (Jones 1992 [DIRS 146405], Tables 1 and 2).

The half-lives of the radionuclides used in the crud source calculations are provided by Nuclides and Isotopes, Chart of the Nuclides (Parrington et al. 1996 [DIRS 103896]).

PWR Lattice-For the PWR commercial SNF, the Babcock \& Wilcox (B\&W) Mark B $15 \times 15$ PWR fuel assembly is selected as the generic PWR lattice for analysis. This lattice has a high initial heavy metal loading and large amounts of stainless steel and Inconel assembly hardware, maximizing fission product generation and activation of structural hardware. While a typical B\&W Mark B assembly has an initial heavy metal loading of $464 \mathrm{~kg}$ of uranium (Punatar 2001 [DIRS 155635], Table 3.1), this is increased to $475 \mathrm{~kg}$ to provide coverage of all commercial SNF waste streams (Assumption 5.1.1.4). Source terms for a generic stainless-steel-clad fuel assembly and for a longer South Texas assembly are also generated in PWR Source Term Generation and Evaluation (BSC 2004 [DIRS 169061]). For the longer South Texas assembly, a uranium metal mass of $550 \mathrm{~kg}$ is used.

The additional uranium mass is accommodated by increasing the fuel length of a B\&W Mark B assembly, rather than by increasing the fuel density. This is consistent with the demonstration of previous calculations that a lower fuel density generates higher gamma and neutron sources described in BWR Source Term Generation and Evaluation (BSC 2003 [DIRS 164364], pp. 48 and 49). A longer active fuel length and a lower density decrease the fuel self-shielding. This results in a higher flux and consequently higher source intensities.

The physical characteristics and operating conditions of the B\&W Mark B $15 \times 15$ PWR fuel assembly are obtained from:

- Summary Report of Commercial Reactor Criticality Data for Crystal River Unit 3 (Punatar 2001 [DIRS 155635], Table 2-2, Figure 2-2, Table 2-9)

- Operational Data—B\&W NSS (Framatome Cogema Fuels 1999 [DIRS 146419], p. 3)

The Mark B and the South Texas assemblies are used in the evaluation of the crud activity on the assembly surface. The larger fuel rod surface area in the South Texas assembly is used as the bounding case because crud activity is directly proportional to the surface area in the assembly exposed to the coolant.

BWR Lattice - For BWR commercial SNF, a General Electric (GE) $8 \times 8$ BWR fuel assembly is used as the generic fuel design for BWR source term determination (BSC 2003 [DIRS 164364], p. 6). This design has a high initial heavy metal loading and an adequate amount of fuel assembly hardware data. The initial heavy metal loading is conservatively increased from $184 \mathrm{~kg}$ (Larsen et al. 1976 [DIRS 146576], p. A-2) to $200 \mathrm{~kg}$ to provide coverage of the actual waste stream (Assumption 5.1.1.4). In cases where the hardware for the assembly is not conservative, substitutions and approximations are made to increase conservatism. The 
stainless-steel-clad fuel assembly is also considered because it presents higher gamma source intensity due to activation.

As was the case for PWR fuel, the additional fuel mass is added as increased length, rather than as increased density.

The crud activity is directly proportional to the surface area in the assembly components exposed to coolant. Therefore, an assembly that has a higher surface area is used to conservatively evaluate the crud activity. The Advanced Nuclear Fuel $9 \times 9 \mathrm{JP}-4$ fuel assembly is selected for crud activity evaluation of a BWR assembly because it has a greater area exposed to coolant than that of the GE $8 \times 8$ BWR fuel assembly.

The physical characteristics and operating conditions for the GE $8 \times 8$ fuel assembly are obtained from the following sources:

- Summary Report of Commercial Reactor Criticality Data for Quad Cities Unit 2 (CRWMS M\&O 1999 [DIRS 134660])

- Core Design and Operating Data for Cycles 1 and 2 of Quad Cities 1 (Larsen et al. 1976 [DIRS 146576], pp. A-1, A-8, and C-12)

- Appendix 2A. Physical Descriptions of LWR Fuel Assemblies. Volume 3 of Characteristics of Spent Fuel, High-Level Waste, and Other Radioactive Wastes Which May Require Long-Term Isolation (DOE 1987 [DIRS 132333], pp. 2A-16 and 2A-158).

Additional information required to perform the BWR source term calculation (BSC 2003 [DIRS 164364]) is taken from:

- CRC Depletion Calculations for Quad Cities Unit 2 (CRWMS M\&O 1999 [DIRS 134650], pp. 50-55) for dimensions and materials for PATH B geometric descriptions of the SAS2H code

- SCALE, RSIC Computer Code Collection (NRC 1997 [DIRS 122675], Table S2.6.4, p. S2.6.12) for clad temperature during operation.

\subsubsection{Inputs for U.S. Department of Energy Spent Nuclear Fuel and High-level Radioactive Waste Source Term Determinations}

The DOE SNF and HLW source term calculations rely on the input of initial radionuclide inventories and, in the case of HLW, chemical compositions of the glass waste forms. The initial radionuclide inventories for DOE SNF are taken from Source Term Estimates for DOE Spent Nuclear Fuels (DOE 2003 [163377]).

The volume, mass, and canister quantities for the HLW have historically been taken from Final Environmental Impact Statement for a Geologic Repository for the Disposal of Spent Nuclear Fuel and High-Level Radioactive Waste at Yucca Mountain, Nye County, Nevada (DOE 2002 [DIRS 155970], Volume II, Appendixes A through O, pp. A-39 to A-43, A-48, A-55, and A-56). 
As inventories are better quantified, the canister quantities are taken from updated references (e.g., Allison 2004 [DIRS 168734]).

Chemical compositions and initial radionuclide inventories are provided by:

- "Response to Repository Environmental Impact Statement Data Call for High-Level Waste" (Picha 1997 [DIRS 104406], pp. 6, 7, and 12)

- "Clarification of High-Level Waste and Special Performance Assessment Required Data for Repository Environmental Impact Statement" (Picha 1998 [DIRS 104407], p. 3)

- Projected Radionuclide Inventories and Radiogenic Properties of the DWPF Product (U) (Plodinec and Marra 1994 [DIRS 101908], p. 10)

- Waste Form Qualification Report (WQR), West Valley Demonstration Project (WVNS 1998 [DIRS 103500], Section WQR-1.1, p. 7 and Section WQR-1.2, pp. AP-1 to AP-3)

- "Revision to Original INEEL Response to Yucca Mountain Site Characterization Office Data Call for High-Level Waste" (Goff 1998 [DIR.S 104389], pp. 3 to 7)

- "Follow Up Response to Repository Environmental Impact Statement Data Call for High-Level Waste" (Picha 1998 [DIRS 104413], p. 9)

- "Response to Clarification Data for the Repository Environmental Impact Statement (EIS) Data Call Memorandum Dated October 3, 1997" (Taylor 1997 [DIRS 104476], pp. 8 to 10 )

\subsubsection{Structural}

The technical data and parameters for structural calculations include the mechanical properties of the design materials and the configuration of the surface facility and engineered barrier system. In addition, the geometry of the waste package, emplacement pallet, and drip shield are used in the process; however, these parameters are varied appropriately to obtain designs that comply with the governing requirements.

\subsubsection{Mechanical Material Properties}

Sources for values of the mechanical material properties used in structural analyses are listed in Table 2. Discussions on the use of some of the material properties listed in this table are provided in Section 5.2.

\subsubsection{Configuration Interfaces}

Coordination drawings define the interfaces for waste package components with facilities and the engineered barrier system. Interface controls that delineate the design responsibilities of the Analysis and Component Design organization and other organizations are developed as necessary. 


\subsubsection{Dimensional and Material Variability}

All structural calculations assume the nominal thicknesses for the inner vessel and corrosion barrier are the minimum material thicknesses. Future drawings will indicate tolerances that show these dimensions as minimum values. This ensures structural design requirements will be achieved.

Available minimum material strength properties are used to ensure conservative assessments due to material variability. When available, material properties that are temperature dependent are used for variable temperature environment calculations. In general, when a range of values is given for material properties, the values that ensure conservative results are used.

Table 2. Sources of Material Properties for Structural Analyses

\begin{tabular}{|c|c|}
\hline Material Property & Source \\
\hline \multicolumn{2}{|r|}{ SA-240 S31600 (SS 316) (Inner vessel) } \\
\hline Density $(\rho)$ & $\begin{array}{l}\text { Standard Practice for Preparing, Cleaning, and Evaluating Corrosion Test } \\
\text { Specimens (ASTM G 1-90 1999 [DIRS 103515], Table X1.1) } \\
\end{array}$ \\
\hline \multirow[t]{2}{*}{ Yield Strength $\left(S_{y}\right)$} & $\begin{array}{l}2001 \text { ASME Boiler and Pressure Vessel Code (includes } 2002 \text { addenda) } \\
\text { (ASME } 2001 \text { [DIRS 158115], Section II, Part D, Table Y-1) }\end{array}$ \\
\hline & $\begin{array}{l}\text { Corrosion. Volume } 13 \text { of Metals Handbook (ASM International } 1987 \text { [DIRS } \\
103753 \text { ], p. 931). This document is used to determine chemical composition of } \\
316 \text { stainless steel }\end{array}$ \\
\hline \multirow[t]{2}{*}{ Uitimate Tensile Strength $\left(\mathrm{S}_{\mathrm{u}}\right)$} & $\begin{array}{l}\text { 2001 ASME Boiler and Pressure Vessel Code (includes } 2002 \text { addenda) } \\
\text { (ASME } 2001 \text { [DIRS 158115], Section II, Part D, Table U) }\end{array}$ \\
\hline & $\begin{array}{l}\text { Corrosion. Volume } 13 \text { of Metals Handbook (ASM International } 1987 \text { [DIRS } \\
103753 \text { ], p. 931). This document is used to determine chemical composition of } \\
316 \text { stainless steel }\end{array}$ \\
\hline \multirow[t]{2}{*}{ Percentage Elongation } & $\begin{array}{l}2001 \text { ASME Boiler and Pressure Vessel Code (includes } 2002 \text { addenda) } \\
\text { (ASME } 2001 \text { [DIRS 158115], Section II, Part A, SA-240, Table 2), which } \\
\text { presents specifications for heat-resisting chromium and chromium-nickel } \\
\text { stainless steel plate, sheet, and strip for pressure vessels }\end{array}$ \\
\hline & $\begin{array}{l}\text { Technical Data Blue Sheet, Stainless Steels, Chromium-Nickel-Molybdenum, } \\
\text { Types } 316 \text { (S31600), 316L (S31603), } 317 \text { (S31700), 317L (S31703) (Allegheny } \\
\text { Ludlum } 1999 \text { [DIRS 151409], p. 8) }\end{array}$ \\
\hline Elastic Modulus (E) & $\begin{array}{l}2001 \text { ASME Boiler and Pressure Vessel Code (includes } 2002 \text { addenda) } \\
\text { (ASME } 2001 \text { [DIRS 158115], Section II, Part D, Table TM-1) }\end{array}$ \\
\hline $\begin{array}{l}\text { Mean Coefficient of Thermal } \\
\text { Expansion }\end{array}$ & $\begin{array}{l}2001 \text { ASME Boiler and Pressure Vessel Code (includes } 2002 \text { addenda) } \\
\text { (ASME } 2001 \text { [DIRS 158115], Section II, Part D, Table TE-1) }\end{array}$ \\
\hline Poisson's Ratio $(\varepsilon)$ & $\begin{array}{l}\text { Properties and Selection: Stainless Steels, Tool Materials and Special- } \\
\text { Purpose Metals. Volume } 3 \text { of Metals Handbook (ASM } 1980 \text { [DIRS 104317], } \\
\text { Figure 15, p. 755) }\end{array}$ \\
\hline \multicolumn{2}{|r|}{ TSw2 Rock } \\
\hline Density $(\rho)$ & $\begin{array}{l}\text { Reference Information Base Data Item: Rock Geomechanical Properties } \\
\text { (DTN: MO9808RIB00041.000 [DIRS 104850], Table 5) }\end{array}$ \\
\hline \multicolumn{2}{|r|}{ SA-312 S31603 (SS 316L) (DOE Canisters) } \\
\hline Density $(\rho)$ & $\begin{array}{l}\text { Standard Practice for Preparing, Cleaning, and Evaluating Corrosion Test } \\
\text { Specimens (ASTM G 1-90 1999 [DIRS 103515], Table X1.1) }\end{array}$ \\
\hline Yield Strength $\left(S_{y}\right)$ & $\begin{array}{l}\text { 2001 ASME Boiler and Pressure Vessel Code (includes } 2002 \text { addenda) } \\
\text { - (ASME } 2001 \text { [DIRS 158115], Section II, Part D, Table Y-1) }\end{array}$ \\
\hline Ultimate Tensile Strength $\left(\mathrm{S}_{u}\right)$ & $\begin{array}{l}2001 \text { ASME Boiler and Pressure Vessel Code (includes } 2002 \text { addenda) } \\
\text { (ASME } 2001 \text { [DIRS 158115], Section II, Part D, Table U) }\end{array}$ \\
\hline Percentage Elongation & $\begin{array}{l}2001 \text { ASME Boiler and Pressure Vessel Code (includes } 2002 \text { addenda) } \\
\text { (ASME } 2001 \text { [DIRS 158115], Section II,.Part A, SA-240. Table 2) }\end{array}$ \\
\hline
\end{tabular}




\begin{tabular}{|l|l|}
\hline Elastic Modulus (E) & $\begin{array}{l}\text { 2001 ASME Boiler and Pressure Vessel Code (includes 2002 addenda) } \\
\text { (ASME 2001 [DIRS 158115], Section II, Part D, Table TM-1) }\end{array}$ \\
\hline Poisson's Ratio ( $(\varepsilon)$ & $\begin{array}{l}\text { Properties and Selection: Stainless Steels, Tool Materials and Special- } \\
\text { Purpose Metals. Volume } 3 \text { of Metals Handbook (ASM 1980 [DIRS 104317], } \\
\text { Figure 15, p. 755). Poisson's ratio for 316 stainless steel will be used for 316L } \\
\text { stainless steel (Assumption 5.2.8.4). }\end{array}$ \\
\hline
\end{tabular}


Table 2. Sources of Material Properties for Structural Analyses (Continued)

\begin{tabular}{|c|c|}
\hline Material Property & Source \\
\hline \multicolumn{2}{|r|}{ SA-705 S017400 (17-4 PH) (Lifting Collars) } \\
\hline Density $(\rho)$ & $\begin{array}{l}\text { Properties and Selection: Stainless Steels, Tool Materials and Special- } \\
\text { Purpose Metals. Volume } 3 \text { of Metals Handbook (ASM } 1980 \text { [DIRS 104317], } \\
\text { Table 12, p. 34) }\end{array}$ \\
\hline Yield Strength $\left(S_{y}\right)$ & $\begin{array}{l}\text { Properties and Selection of Metals. Volume } 1 \text { of Metals Handbook (ASM } 1961 \\
\text { [DIRS 158132], p. 506) }\end{array}$ \\
\hline Ultimate Tensile Strength $\left(S_{u}\right)$ & $\begin{array}{l}\text { Properties and Selection of Metals. Volume } 1 \text { of Metals Handbook (ASM } 1961 \\
\text { DIRS } 158132 \text { p. 506) }\end{array}$ \\
\hline Percentage Elongation & $\begin{array}{l}2001 \text { ASME Boiler and Pressure Vessel Code (includes } 2002 \text { addenda) (ASME } \\
2001 \text { [DIRS 158115], Section II, Part A, SA-705/SA-705M, Table 3) }\end{array}$ \\
\hline Elastic Modulus (E) & $\begin{array}{l}2001 \text { ASME Boiler and Pressure Vessel Code (includes } 2002 \text { addenda) (ASME } \\
2001 \text { [DIRS 158115], Section II, Part D, Table TM-1) }\end{array}$ \\
\hline Poisson's Ratio $(\varepsilon)$ & "17-4Ph Technical Data" (High Temp Metals 2002 DIRS [158006]) \\
\hline \multicolumn{2}{|c|}{ SA-240 S30400 (SS 304) (Fuel Assembly Components) } \\
\hline Yield Strength $\left(S_{y}\right)$ & $\begin{array}{l}2001 \text { ASME Boiler and Pressure Vessel Code (includes } 2002 \text { addenda) } \\
\text { (ASME } 2001 \text { [DIRS 158115], Section II, Part D, Table Y-1) }\end{array}$ \\
\hline Ultimate Tensile Strength $\left(\mathbf{S}_{\mathrm{u}}\right)$ & $\begin{array}{l}2001 \text { ASME Boiler and Pressure Vessel Code (includes } 2002 \text { addenda) } \\
\text { (ASME } 2001 \text { [DIRS 158115], Section II, Part D, Table U) }\end{array}$ \\
\hline Elastic Modulus (E) & $\begin{array}{l}2001 \text { ASME Boiler and Pressure Vessel Code (includes } 2002 \text { addenda) } \\
\text { (ASME } 2001 \text { [DIRS 158115], Section II, Part D, Table TM-1) }\end{array}$ \\
\hline Poisson's Ratio $(\varepsilon)$ & $\begin{array}{l}\text { Properties and Selection: Stainless Steels, Tool Materials and Special- } \\
\text { Purpose Metals. Volume } 3 \text { of Metals Handbook (ASM } 1980 \text { [DIRS 104317], } \\
\text { Figure 15, p. 755) }\end{array}$ \\
\hline \multicolumn{2}{|c|}{ SA-516 K02700 (A 516, Grade 70) (Fuel Tubes, Structural Plates) } \\
\hline Density $(\rho)$ & $\begin{array}{l}2001 \text { ASME Boiler and Presisure Vessel Code (includes } 2002 \text { addenda) } \\
\text { (ASME } 2001 \text { [DIRS 158115], Section II, Part A, SA-20, Section 14.1), which } \\
\text { presents specifications for presisure vessel plates, carbon steel, for moderate- } \\
\text { and lower-temperature service }\end{array}$ \\
\hline Yield Strength $\left(\mathrm{S}_{\mathrm{y}}\right)$ & $\begin{array}{l}2001 \text { ASME Boiler and Pressure Vessel Code (includes } 2002 \text { addenda) } \\
\text { (ASME } 2001 \text { [DIRS 158115], Section II, Part D, Table Y-1) }\end{array}$ \\
\hline Ultimate Tensile Strength $\left(\mathrm{S}_{u}\right)$ & $\begin{array}{l}2001 \text { ASME Boiler and Pressure Vessel Code (includes } 2002 \text { addenda) } \\
\text { (ASME } 2001 \text { [DIRS 158115], Section II, Part D, Table U) }\end{array}$ \\
\hline Percentage Elongation & $\begin{array}{l}2001 \text { ASME Boiler and Pressure Vessel Code (includes } 2002 \text { addenda) } \\
\text { (ASME } 2001 \text { [DIRS 158115], Section II, Part A, SA-516, Table 2) }\end{array}$ \\
\hline Elastic Modulus (E) & $\begin{array}{l}2001 \text { ASME Boiler and Pressure Vessel Code (includes } 2002 \text { addenda) } \\
\text { (ASME } 2001 \text { [DIRS 158115], Section II, Part D, Table TM-1) }\end{array}$ \\
\hline $\begin{array}{l}\text { Mean Coefficient of Thermal } \\
\text { Expansion }\end{array}$ & $\begin{array}{l}2001 \text { ASME Boiler and Pressure Vessel Code (includes } 2002 \text { addenda) } \\
\text { (ASME } 2001 \text { [DIRS 158115], Section II, Part D, Table TE-1) }\end{array}$ \\
\hline Poisson's Ratio $(\varepsilon)$ & $\begin{array}{l}\text { Properties and Selection: Irons and Steels. Volume } 1 \text { of Metals Handbook } \\
\text { (ASM } 1978 \text { [DIRS 102018], p. 393), which provides Poisson's ratio for cast } \\
\text { carbon steel that will be used for SA-516 carbon steel (Assumption 5.2.8.3) }\end{array}$ \\
\hline \multicolumn{2}{|r|}{ SB-209 A96061 T4 (Alloy 6061 T4) (Thermal Shunts) } \\
\hline Density $(\rho)$ & $\begin{array}{l}2001 \text { ASME Boiler and Pressure Vessel Code (includes } 2002 \text { addenda) } \\
\text { (ASME 2001 [DIRS 158115], Section II, Part D, Table NF-2) }\end{array}$ \\
\hline Yield Strength $\left(S_{y}\right)$ & $\begin{array}{l}2001 \text { ASME Boiler and Pressure Vessel Code (includes } 2002 \text { addenda) } \\
\text { (ASME } 2001 \text { [DIRS 158115], Section II, Part B, SB-209, Table 3), which } \\
\text { provides specifications for aluminum and aluminum-alloy sheet and plate }\end{array}$ \\
\hline Ultimate Tensile Strength $\left(S_{u}\right)$ & $\begin{array}{l}2001 \text { ASME Boiler and Pressure Vessel Code (includes } 2002 \text { addenda) } \\
\text { (ASME } 2001 \text { [DIRS 158115], Section II, Part B, SB-209, Table 3), which } \\
\text { provides specifications for aluminum and aluminum-alloy sheet and plate }\end{array}$ \\
\hline Percentage Elongation & $\begin{array}{l}2001 \text { ASME Boiler and Pressure Vessel Code (includes } 2002 \text { addenda) } \\
\text { (ASME } 2001 \text { [DIRS } 158115 \text { ], Section II, Part B, SB-209, Table 3), which } \\
\text { provides specifications for aluminum and aluminum-alloy sheet and plate }\end{array}$ \\
\hline
\end{tabular}


Table 2. Sources of Material Properties for Structural Analyses (Continued)

\begin{tabular}{|c|c|}
\hline Material Property & Source \\
\hline Elastic Modulus (E) & $\begin{array}{l}2001 \text { ASME Boiler and Pressure Vessel Code (includes } 2002 \text { addenda) } \\
\text { (ASME } 2001 \text { [DIRS 158115], Section II, Part D, Table TM-2) }\end{array}$ \\
\hline $\begin{array}{l}\text { Mean Coefficient of Thermal } \\
\text { Expansion }\end{array}$ & $\begin{array}{l}2001 \text { ASME Boiler and Pressure Vessel Code (includes } 2002 \text { addenda) } \\
\text { (ASME } 2001 \text { [DIRS 158115], Section II, Part D, Table TE-2) }\end{array}$ \\
\hline Poisson's Ratio $(\varepsilon)$ & $\begin{array}{l}2001 \text { ASME Boiler and Pressure Vessel Code (includes } 2002 \text { addenda) } \\
\text { (ASME 2001 [DIRS 158115], Section II, Part D, Table NF-1) }\end{array}$ \\
\hline \multicolumn{2}{|r|}{ SB-265 R52400 (Ti Grade 7) (Drip Shield) } \\
\hline Density $(\rho)$ & $\begin{array}{l}2001 \text { ASME Boiler and Pressure Vessel Code (includes } 2002 \text { addenda) } \\
\text { (ASME } 2001 \text { [DIRS 158115], Section II, Part D, Table NF-2) } \\
\text { Properties and Selection: Norferrous Alloys and Special-Purpose Materials. } \\
\text { Volume } 2 \text { of ASM Handbook (ASM International } 1990 \text { [DIRS 141615], Table } \\
20, \text { p. 620) }\end{array}$ \\
\hline Yield Strength $\left(S_{y}\right)$ & $\begin{array}{l}2001 \text { ASME Boiler and Pressure Vessel Code (includes } 2002 \text { addenda) } \\
\text { (ASME } 2001 \text { [DIRS 158115], Section II, Part D, Table Y-1) }\end{array}$ \\
\hline Ultimate Tensile Strength $\left(\mathbf{S}_{\mathrm{u}}\right)$ & $\begin{array}{l}2001 \text { ASME Boiler and Pressure Vessel Code (includes } 2002 \text { addenda) } \\
\text { (ASME } 2001 \text { [DIRS } 158115 \text { ], Section II, Part B, SB-265, Table 1), which } \\
\text { provides specifications for titarium and titanium alloy strip, sheet, and plate }\end{array}$ \\
\hline Percentage Elongation & $\begin{array}{l}2001 \text { ASME Boiler and Pressure Vessel Code (includes } 2002 \text { addenda) } \\
\text { (ASME } 2001 \text { [DIRS } 158115 \text { ], Section II, Part B, SB-265, Table 1), which } \\
\text { provides specifications for titanium and titanium alloy strip, sheet, and plate }\end{array}$ \\
\hline Elastic Modulus (E) & $\begin{array}{l}2001 \text { ASME Boiler and Pressure Vessel Code (includes } 2002 \text { addenda) } \\
\text { (ASME } 2001 \text { [DIRS 158115], Section II, Part D, Table TM-5) }\end{array}$ \\
\hline $\begin{array}{l}\text { Mean Coefficient of Thermal } \\
\text { Expansion }\end{array}$ & $\begin{array}{l}2001 \text { ASME Boiler and Pressure Vessel Code (includes } 2002 \text { addenda) } \\
\text { (ASME } 2001 \text { [DIRS 158115], Siection II, Part D, Table TE-5) }\end{array}$ \\
\hline \multirow[t]{2}{*}{ Poisson's Ratio $(\varepsilon)$} & $\begin{array}{l}2001 \text { ASME Boiler and Pressure Vessel Code (includes } 2002 \text { addenda) } \\
\text { (ASME } 2001 \text { [DIRS 158115], Section II, Part D, Table NF-1) }\end{array}$ \\
\hline & $\begin{array}{l}\text { Properties and Selection: Nonferrous Alloys and Special-Purpose Materials. } \\
\text { Volume } 2 \text { of ASM Handbook (ASM International } 1990 \text { [DIRS 141615], Table } \\
\text { 21, p. 621) }\end{array}$ \\
\hline \multicolumn{2}{|c|}{ SB-265 R56405 (Ti Grade 24) (Drip Shield Structural Members) } \\
\hline Density $(\rho)$ & $\begin{array}{l}\text { Properties and Selection: Nonferrous Alloys and Special-Purpose Materials. } \\
\text { Volume } 2 \text { of ASM Handbook (A.SM International } 1990 \text { [DIRS 141615], Table } \\
\text { 20, p. 620). }\end{array}$ \\
\hline Yield Strength $\left(S_{y}\right)$ & $\begin{array}{l}2001 \text { ASME Boiler and Pressure Vessel Code (includes } 2002 \text { addenda) } \\
\text { (ASME } 2001 \text { [DIRS 158115], Section II, Part B, SB-265, Table 3), which } \\
\text { provides specifications for titanium and titanium alloy strip, sheet, and plate }\end{array}$ \\
\hline Ultimate Tensile Strength $\left(S_{u}\right)$ & $\begin{array}{l}2001 \text { ASME Boiler and Pressure Vessel Code (includes } 2002 \text { addenda) } \\
\text { (ASME } 2001 \text { [DIRS 158115], Section II, Part B, SB-265, Table 1), which } \\
\text { provides specifications for titanium and titanium alloy strip, sheet, and plate }\end{array}$ \\
\hline Percentage Elongation & $\begin{array}{l}2001 \text { ASME Boiler and Pressure Vessel Code (includes } 2002 \text { addenda) } \\
\text { (ASME } 2001 \text { [DIRS 158115], Section II, Part B, SB-265, Table 1) }\end{array}$ \\
\hline Elastic Modulus (E) & $\begin{array}{l}\text { Properties and Selection: Nonierrous Alloys and Special-Purpose Materials. } \\
\text { Volume } 2 \text { of ASM Handbook (ASM International } 1990 \text { [DIRS 141615], Table } \\
21, \text { p. } 621 \text { ) }\end{array}$ \\
\hline $\begin{array}{l}\text { Mean Coefficient of Thermal } \\
\text { Expansion }\end{array}$ & $\begin{array}{l}\text { Properties and Selection: Nonierrous Alloys and Special-Purpose Materials. } \\
\text { Volume } 2 \text { of ASM Handbook (A.SM International } 1990 \text { [DIRS 141615], Table } \\
20, \text { p. } 620 \text { ) }\end{array}$ \\
\hline Poisson's Ratio $(\varepsilon)$ & $\begin{array}{l}\text { Properties and Selection: Nonierrous Alloys and Special-Purpose Materials. } \\
\text { Volume } 2 \text { of ASM Handbook (A.SM International } 1990 \text { [DIRS 141615], Table } \\
21, \text { p. } 621 \text { ). }\end{array}$ \\
\hline
\end{tabular}


Table 2. Sources of Material Properties for Structural Analyses (Continued)

\begin{tabular}{|c|c|}
\hline Material Property & Source \\
\hline \multicolumn{2}{|c|}{ Alloy 22 N06022 (SB-575 N06022) (Outer Corrosion Barrier, Emplacement Pallet Plates) } \\
\hline Density $(\rho)$ & $\begin{array}{l}2001 \text { ASME Boiler and Pressure Vessel Code (includes } 2002 \text { addenda) (ASME } \\
2001 \text { [DIRS 158115], Section II, Part B, SB-575, Section 7.1), which provides } \\
\text { specifications for low-carbon nickel-molybdenum-chromium, low-carbon nickel- } \\
\text { chromium-molybdenum, low-carbon nickel-chromium-molybdenum-copper, and } \\
\text { low-carbon nickel-chromium-molybdenum-tungsten alloy plate, sheet, and strip }\end{array}$ \\
\hline Yield Strength $\left(S_{y}\right)$ & $\begin{array}{l}2001 \text { ASME Boiler and Pressure Vessel Code (includes } 2002 \text { addenda) (ASME } \\
2001 \text { [DIRS 1581 15], Section II, Part D, Table Y-1) }\end{array}$ \\
\hline Ultimate Tensile Strength $\left(S_{u}\right)$ & $\begin{array}{l}2001 \text { ASME Boiler and Pressure Vessel Code (includes } 2002 \text { addenda) (ASME } \\
2001 \text { [DIRS 158115], Section II, Part D, Table U) } \\
\end{array}$ \\
\hline Percentage Elor & $\begin{array}{l}2001 \text { ASME Boiler and Pressure Vessel Code (includes } 2002 \text { addenda) (ASME } \\
2001 \text { [DIRS 158115], SB-575, Table 3), which provides specifications for low- } \\
\text { carbon nickel-molybdenum-chromium, low-carbon nickel-chromium- } \\
\text { molybdenum, low-carbon nickel-chromium-molybdenum-copper, and low- } \\
\text { carbon nickel-chromium-molybdenum-tungsten alloy plate, sheet, and strip }\end{array}$ \\
\hline & Hastelloy C-22 Alloy (Haynes International 1997 [DIF \\
\hline Elastic Modulus (E) & Hastelloy C-22 Alloy (Haynes International 1997 [DIR \\
\hline $\begin{array}{l}\text { Mean Coefficient of T } \\
\text { Expansion }\end{array}$ & Hastelloy C-22 Alloy (Haynes International 1997 [DIRS 100896], p. 13) \\
\hline Poisson's Ratio $(\varepsilon)$ & $\begin{array}{l}\text { Properties and Selection: Stainless Steels, Tool Materials and Special- } \\
\text { Purpose Metals. Volume } 3 \text { of Metals Handbook (ASM } 1980 \text { [DIRS 104317], p. } \\
\text { 143). Poisson's ratio for Alloy } 625 \text { is used for Alloy } 22 \text { N06022 (Assumption } \\
\text { 5.2.8.2) }\end{array}$ \\
\hline & y C-22 Alloy (Haynes \\
\hline Specific Heat $\left(c_{p}\right)$ & Hastelloy C-22 Alloy (Haynes International 1997 [DIRS 100896], p. 13) \\
\hline \multicolumn{2}{|c|}{$\begin{array}{l}\text { Neutronit A } 978 \text { (Neutron Absorber Plates) } \\
\text { NOTE: At the time of this revision a new material (a nickel-gadilium alloy) has just been selected for } \\
\text { absorber plates. Future revisions to this docuinent will make the change. }\end{array}$} \\
\hline Density $(\rho)$ & $\begin{array}{l}\text { Sheet and Plate for Nuclear Engineering, BÖHLER Neutronit A976 (Kügler } \\
1997 \text { [DIRS 134327], p. 17). The density of Neutronit A } 976 \text { is used for } \\
\text { Neutronit A } 978 \text { (Assumption 5.2.8.11) }\end{array}$ \\
\hline Yield Stren & $\begin{array}{l}\text { Sheet and Plate for Nuclear Engineering, BÖHLER Neutronit A976 (Kügler } \\
1997 \text { [DIRS 134327], p. 15). The yield strength of Neutronit A } 976 \text { is used for } \\
\text { Neutronit A 978 (Assumption 5.2.8.11) }\end{array}$ \\
\hline Ultimate Tensile Streng & $\begin{array}{l}\text { Sheet and Plate for Nuclear Engineering, BÖHLER Neutronit A976 (Kügler } \\
1997 \text { [DIRS 134327], p. 15). The ultimate strength of Neutronit A } 976 \text { is used } \\
\text { for Neutronit A 978 (Assumption 5.2.8.11) }\end{array}$ \\
\hline Percentage Elongation & $\begin{array}{l}\text { Sheet and Plate for Nuclear Engineering, BÖHLER Neutronit A976 (Kügler } \\
1997 \text { [DIRS 134327], p. 15). The percentage elongation of Neutronit A } 976 \text { is } \\
\text { used for Neutronit A } 978 \text { (Assumption 5.2.8.11) }\end{array}$ \\
\hline Elastic Modulus (E) & $\begin{array}{l}\text { Sheet and Plate for Nuclear Engineering, BÖHLER Neutronit A976 (Kügler } \\
1997 \text { [DIRS 134327], p. 17). The elastic modulus of Neutronit A } 976 \text { is used for } \\
\text { Neutronit A } 978 \text { (Assumption 5.2.8.11) }\end{array}$ \\
\hline $\begin{array}{l}\text { Mean Coefficient of The } \\
\text { Expansion }\end{array}$ & $\begin{array}{l}2001 \text { ASME Boiler and Pressure Vessel Code (includes } 2002 \text { addenda) (ASME } \\
2001 \text { [DIRS 158115], Section II, Part D, Table TE-1). The coefficient of thermal } \\
\text { expansion of Neutronit A } 976 \text { is used for Neutronit A } 978 \text { (Assumption 5.2.8.11) }\end{array}$ \\
\hline Poisson's Ratio $(\varepsilon)$ & $\begin{array}{l}\text { Properties and Selection: Stainless Steels, Tool Materials and Special- } \\
\text { Purpose Metals. Volume } 3 \text { of Metals Handbook (ASM } 1980 \text { [DIRS 104317], } \\
\text { Figure 15, p. 755). Poisson's ratio for Neutronit A } 976 \text { is used for Neutronit A } \\
978 \text { (Assumption 5.2.8.11) }\end{array}$ \\
\hline
\end{tabular}

\subsubsection{Thermal}

As is the case for structural analyses, the engineering designs of the waste package, emplacement pallet, drip shield, and surface facilities are also input to the thermal analyses. In addition, the 
thermal properties for the design materials and the configuration of the natural system are needed for thermal analyses.

\subsubsection{Thermal Material Properties}

The material properties used in thermal analyses are fourd in the references listed in Table 3 for the major materials used in the design.

Table 3. Sources of Material Properties for Thermal Analyses

\begin{tabular}{|c|c|}
\hline Material Property & Source \\
\hline \multicolumn{2}{|r|}{ SA-240 S31600 (SS 316) (Inner Vessel) } \\
\hline Density $(\rho)$ & $\begin{array}{l}\text { Standard Practice for Preparing, Cleaning, and Evaluating Corrosion Test } \\
\text { Specimens (ASTM G 1-90 1999 [DIRS 103515], Table X1.1) }\end{array}$ \\
\hline Emissivity $(\varepsilon)$ & $\begin{array}{l}\text { Marks' Standard Handbook for Mechanical Engineers (Avallone and } \\
\text { Baumeister } 1987 \text { [DIRS 103508], p. 4-68) }\end{array}$ \\
\hline Thermal Conductivity (k) & $\begin{array}{l}\text { 2001 ASME Boiler and Pressiure Vessel Code (includes } 2002 \text { addenda) } \\
\text { (ASME } 2001 \text { [DIRS 158115], Section II, Part D, Table TCD) }\end{array}$ \\
\hline Specific Heat $\left(c_{p}\right)$ & $\begin{array}{l}\text { 2001 ASME Boiler and Pressiure Vessel Code (includes } 2002 \text { addenda) } \\
\text { (ASME } 2001 \text { [DIRS 158115], Section II, Part D, Table TCD)-computed from } \\
\text { the thermal diffusivity }\end{array}$ \\
\hline \multicolumn{2}{|c|}{ SB-575 N06022 (Alloy 22 N06022) (Outer Corrosion Barrier) } \\
\hline Density $(\rho)$ & $\begin{array}{l}2001 \text { ASME Boiler and Pressure Vessel Code (includes } 2002 \text { addenda) } \\
\text { (ASME } 2001 \text { [DIRS 158115], Section II, Part B, SB-575, Section 7.1), which } \\
\text { provides specifications for low-carbon nickel-molybdenum-chromium, low- } \\
\text { carbon nickel-chromium-molybdenum, low-carbon nickel-chromium- } \\
\text { molybdenum-copper, and low-carbon nickel-chromium-molybdenum- } \\
\text { tungsten alloy plate, sheet, and strip }\end{array}$ \\
\hline Emissivity $(\varepsilon)$ & $\begin{array}{l}\text { CRC Handbook of Chemistry and Physics (Lide } 1995 \text { [DIRS 101876], p. 10- } \\
\text { 297) for nickel-chromium alloy }\end{array}$ \\
\hline Thermal Conductivity $(\mathrm{k})$ & Hastelloy C-22 Alloy (Haynes International 1997 [DIRS 100896], p. 13) \\
\hline Specific Heat $\left(c_{p}\right)$ & Hastelloy C-22 Alloy (Haynes International 1997 [DIRS 100896], p. 13) \\
\hline \multicolumn{2}{|c|}{ SB-265 R52400 (Ti Grade 7, also used for Ti Grade 24) (Drip Shield) } \\
\hline Density $(\rho)$ & $\begin{array}{l}\text { 2001 ASME Boiler and Pressiure Vessel Code (includes } 2002 \text { addenda) } \\
\text { (ASME } 2001 \text { [DIRS 158115], Section II, Part D, Table NF-2) }\end{array}$ \\
\hline Emissivity $(\varepsilon)$ & 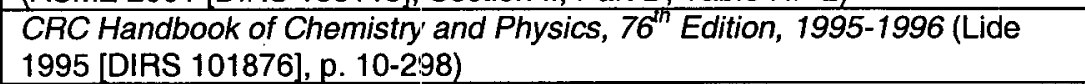 \\
\hline Thermal Conductivity ( $k$ ) & $\begin{array}{l}2001 \text { ASME Boiler and Pressure Vessel Code (includes } 2002 \text { addenda) } \\
\text { (ASME } 2001 \text { [DIRS 158115], Section II, Part D, Table TCD) }\end{array}$ \\
\hline Specific Heat $\left(c_{p}\right)$ & $\begin{array}{l}2001 \text { ASME Boiler and Pressure Vessel Code (includes } 2002 \text { addenda) } \\
\text { (ASME } 2001 \text { [DIRS 158115], Section II, Part D, Table TCD) computed from } \\
\text { the thermal diffusivity }\end{array}$ \\
\hline \multicolumn{2}{|c|}{ SB-209 A96061 T4 (Alloy 6061 T4) and SB-209 A96061 T451 (Alloy 6061 T451) (Thermal Shunts) } \\
\hline Density $(\rho)$ & $\begin{array}{l}2001 \text { ASME Boiler and Pressure Vessel Code (includes } 2002 \text { addenda) } \\
\text { (ASME } 2001 \text { [DIRS 158115], Section II, Part D, Table NF-2) }\end{array}$ \\
\hline Emissivity $(\varepsilon)$ & $\begin{array}{l}\text { Marks' Standard Handbook for Mechanical Engineers (Avallone and } \\
\text { Baumeister 1987 [DIRS 103508], p. 4-68) }\end{array}$ \\
\hline Thermal Conductivity $(\mathrm{k})$ & $\begin{array}{l}2001 \text { ASME Boiler and Pressure Vessel Code (includes } 2002 \text { addenda) } \\
\text { (ASME } 2001 \text { [DIRS 158115], Section II, Part D, Table TCD) }\end{array}$ \\
\hline Specific Heat $\left(c_{p}\right)$ & $\begin{array}{l}2001 \text { ASME Boiler and Pressure Vessel Code (includes } 2002 \text { addenda) } \\
\text { (ASME } 2001 \text { [DIRS 158115], Section II, Part D, Table TCD) computed from } \\
\text { the thermal diffusivity }\end{array}$ \\
\hline \multicolumn{2}{|r|}{ Neutronit A 978 (Neutron Absorber Plates) } \\
\hline Density $(\rho)$ & $\begin{array}{l}\text { Sheet and Plate for Nuclear Engineering, BÖHLER Neutronit A976 (Kügler } \\
1997 \text { [DIRS 134327], p. 17) }\end{array}$ \\
\hline Emissivity $(\varepsilon)$ & $\begin{array}{l}\text { Marks' Standard Handbook for Mechanical Engineers (Avallone and } \\
\text { Baumeister 1987 [DIRS 103508], p. 4-68). The value for stainless steel } 316 \\
\text { is used. }\end{array}$ \\
\hline
\end{tabular}


Table 3. Sources of Material Properties for Thermal Analyses (Continued)

\begin{tabular}{|c|c|}
\hline Material Property & Source \\
\hline Thermal Conductivity $(\mathrm{k})$ & $\begin{array}{l}\text { Sheet and Plate for Nuclear Engineering, BÖHLER Neutronit A976 (Kügler } \\
1997 \text { [DIRS 134327], p. 17) }\end{array}$ \\
\hline Specific Heat $\left(c_{p}\right)$ & $\begin{array}{l}\text { Sheet and Plate for Nuclear Engineering, BÖHLER Neutronit A976 (Kügler } \\
1997 \text { [DIRS 134327], p. 17) }\end{array}$ \\
\hline \multicolumn{2}{|c|}{ SA-516 K02700 (A 516, Grade 70) (Fuel Assembly Tubes, Basket Guides) } \\
\hline Density $(\rho)$ & $\begin{array}{l}2001 \text { ASME Boiler and Pressure Vessel Code (includes } 2002 \text { addenda) } \\
\text { (ASME } 2001 \text { [DIRS 158115], Section II, Part A, SA-20, Section 14.1), which } \\
\text { provides specifications for general requirements for steel plates for pressure } \\
\text { vessels }\end{array}$ \\
\hline Emissivity $(\varepsilon)$ & $\begin{array}{l}\text { Marks' Standard Handbook for Mechanical Engineers (Avallone and } \\
\text { Baumeister } 1987 \text { [DIRS 103508], Table 4.3.2) }\end{array}$ \\
\hline Thermal Conductivity (k) & $\begin{array}{l}2001 \text { ASME Boiler and Pressure Vessel Code (includes } 2002 \text { addenda) } \\
\text { (ASME } 2001 \text { [DIRS 158115], Section II, Part D, Table TCD) }\end{array}$ \\
\hline Specific Heat $\left(c_{p}\right)$ & $\begin{array}{l}2001 \text { ASME Boiler and Pressure Vessel Code (includes } 2002 \text { addenda) } \\
\text { (ASME } 2001 \text { [DIRS 158115], Section II, Part D, Table TCD) }\end{array}$ \\
\hline \multicolumn{2}{|r|}{ Uranium Dioxide (Fuel) } \\
\hline Density $(\rho)$ & $\begin{array}{l}\text { Nuclear Systems l: Thermal Hydraulic Fundamentals (Todreas and Kazimi } \\
1990 \text { [DIRS 107735], p. 296) }\end{array}$ \\
\hline Emissivity $(\varepsilon)$ & $\begin{array}{l}\text { MATPRO-Version } 11 \text { (Revision 2), A Handbook of Materials Properties for } \\
\text { Use in the Analysis of Light Water Reactor Fuel Rod Behavior (Hagrman et } \\
\text { al. } 1981 \text { [DIRS 103522], Equation A-3.2, p. 48) }\end{array}$ \\
\hline Thermal Conductivity $(k)$ & $\begin{array}{l}\text { Nuclear Systems I: Thermal Hydraulic Fundamentals (Todreas and Kazimi } \\
1990 \text { [DIRS 107735], pp. } 302-303 \text {, calculated for } 95 \% \text { theoretical density) }\end{array}$ \\
\hline Specific Heat $\left(c_{p}\right)$ & $\begin{array}{l}\text { Nuclear Systems I: Thermal Hydraulic Fundamentals (Todreas and Kazimi } \\
1990 \text { [DIRS 107735], p. 296) }\end{array}$ \\
\hline \multicolumn{2}{|r|}{ Zircaloy-2 (Fuel Rod Claclding) } \\
\hline Density $(\rho)$ & $\begin{array}{l}\text { Properties and Selection: Nonferrous Alloys and Special-Purpose Materials } \\
\text { (ASM International } 1990 \text { [DIRS 141615], p. 666) }\end{array}$ \\
\hline Emissivity $(\varepsilon)$ & $\begin{array}{l}\text { MATPRO-Version } 11 \text { (Revision 2), A Handbook of Materials Properties for } \\
\text { Use in the Analysis of Light Water Reactor Fuel Rod Behavior (Hagrman et } \\
\text { al. } 1981 \text { [DIRS 103522], Equation B-3.1b, p. } 230 \text {, calculated as a function of } \\
\text { zirconium oxide layer thickness }\end{array}$ \\
\hline Thermal Conductivity (k) & $\begin{array}{l}\text { MATPRO-Version } 11 \text { (Revision 2), A Handbook of Materials Properties for } \\
\text { Use in the Analysis of Light Water Reactor Fuel Rod Behavior (Hagrman et } \\
\text { al. } 1981 \text { [DIRS 103522], Equation B-2.1, p. 217) }\end{array}$ \\
\hline Specific Heat $\left(c_{p}\right)$ & $\begin{array}{l}\text { Nuclear Systems I: Thermal Hydraulic Fundamentals (Todreas and Kazimi } \\
1990 \text { [DIRS 107735], p. 296) }\end{array}$ \\
\hline \multicolumn{2}{|r|}{ Helium (Fuel Rod and Waste Package Fill Gas) } \\
\hline Density $(\rho)$ & $\begin{array}{l}\text { 2001 ASHRAE Handbook, Fundamentals (ASHRAE } 2001 \text { [DIRS 157789], p. } \\
\text { 19.71) }\end{array}$ \\
\hline Thermal Conductivity (k) & $\begin{array}{l}\text { 2001 ASHRAE Handbook, Fundamentals (ASHRAE } 2001 \text { [DIRS 157789], p. } \\
\text { 19.71) }\end{array}$ \\
\hline Specific Heat $\left(c_{p}\right)$ & $\begin{array}{l}\text { 2001 ASHRAE Handbook, Fundamentals (ASHRAE } 2001 \text { [DIRS 157789], p. } \\
\text { 19.71) }\end{array}$ \\
\hline
\end{tabular}


Table 3. Sources of Material Properties for Thermal Analyses (Continued)

\begin{tabular}{|c|c|}
\hline Material Property & Source \\
\hline \multicolumn{2}{|r|}{ Crushed Tuff (Invert Ballast) } \\
\hline Density $(\rho)$ & $\begin{array}{l}\text { Thermal Properties Measured 12/01/99 to 12/02/99 Using the Thermolink } \\
\text { Soil Multimeter and Thermal Properties Sensor on Selected Potential } \\
\text { Candidate Backfill Materials Used in the Engineered Barrier System (DTN: } \\
\text { GS000483351030.003 [DIRS; 152932]) }\end{array}$ \\
\hline Emissivity $(\varepsilon)$ & $\begin{array}{l}\text { Introduction to Heat Transfer. (Incropera and DeWitt } 1996 \text { [DIRS 107784], } \\
\text { p. } 768 \text { ). Use quartz sand as surrogate. }\end{array}$ \\
\hline Thermal Conductivity (k) & $\begin{array}{l}\text { Thermal Properties Measured 12/01/99 to 12/02/99 Using the Thermolink } \\
\text { Soil Multimeter and Thermal Properties Sensor on Selected Potential } \\
\text { Candidate Backfill Materials Used in the Engineered Barrier System (DTN: } \\
\text { GS000483351030.003 [DIRS 152932]) }\end{array}$ \\
\hline Specific Heat $\left(c_{p}\right)$ & $\begin{array}{l}\text { Thermal Properties Measured 12/01/99 to } 12 / 02 / 99 \text { Using the Thermolink } \\
\text { Soil Multimeter and Thermal Properties Sensor on Selected Potential } \\
\text { Candidate Backfill Materials Used in the Engineered Barrier System (DTN: } \\
\text { GS000483351030.003 [DIRS 152932]) }\end{array}$ \\
\hline
\end{tabular}

\subsubsection{Configuration of the Natural System and Thermal Transport Properties Thereof}

The layout of the subsurface area is shown in Figure 3, taken from Underground Layout Configuration (BSC 2003 [DIRS 165572], Figure 5). The emplacement drifts will be located within the lower part of the lithophysal zone of the densely welded devitrified lithophysal-rich tuff (TSw1) unit and the entire densely welded devitrified lithophysal-poor tuff (TSw2) unit of the Topopah Spring Tuff (BSC 2003 [DIRS 165572], Table II-2). The rock layer thicknesses are measured at the location of $\mathrm{N} 233,760 \mathrm{~m}$ and $\mathrm{E} 170,750 \mathrm{~m}$; the ground surface elevation is $4,663 \mathrm{ft}(1421.3 \mathrm{~m})$.

The stratigraphy of the major geologic units near the center of the repository, and characteristic thermal transport properties rock, are taken from:

Revised Heat Capacity of Yucca Mountain Stratigraphic Units (DTN: SN0307T0510902.003 [DIRS 164196]) for heat capacity of all rock layers

Revised Thermal Conductivity of the Non-Repository Layers of Yucca Mountain (DTN: SN0303T0503102.008 [DIRS 162401]) for clensity and thermal conductivity of non-repository layers

Thermal Conductivity of the Potential Repository Horizon Rev 3 (DTN: SN0404T0503102.011 [DIRS 169129]) for density and thermal conductivity of Repository Layers. 


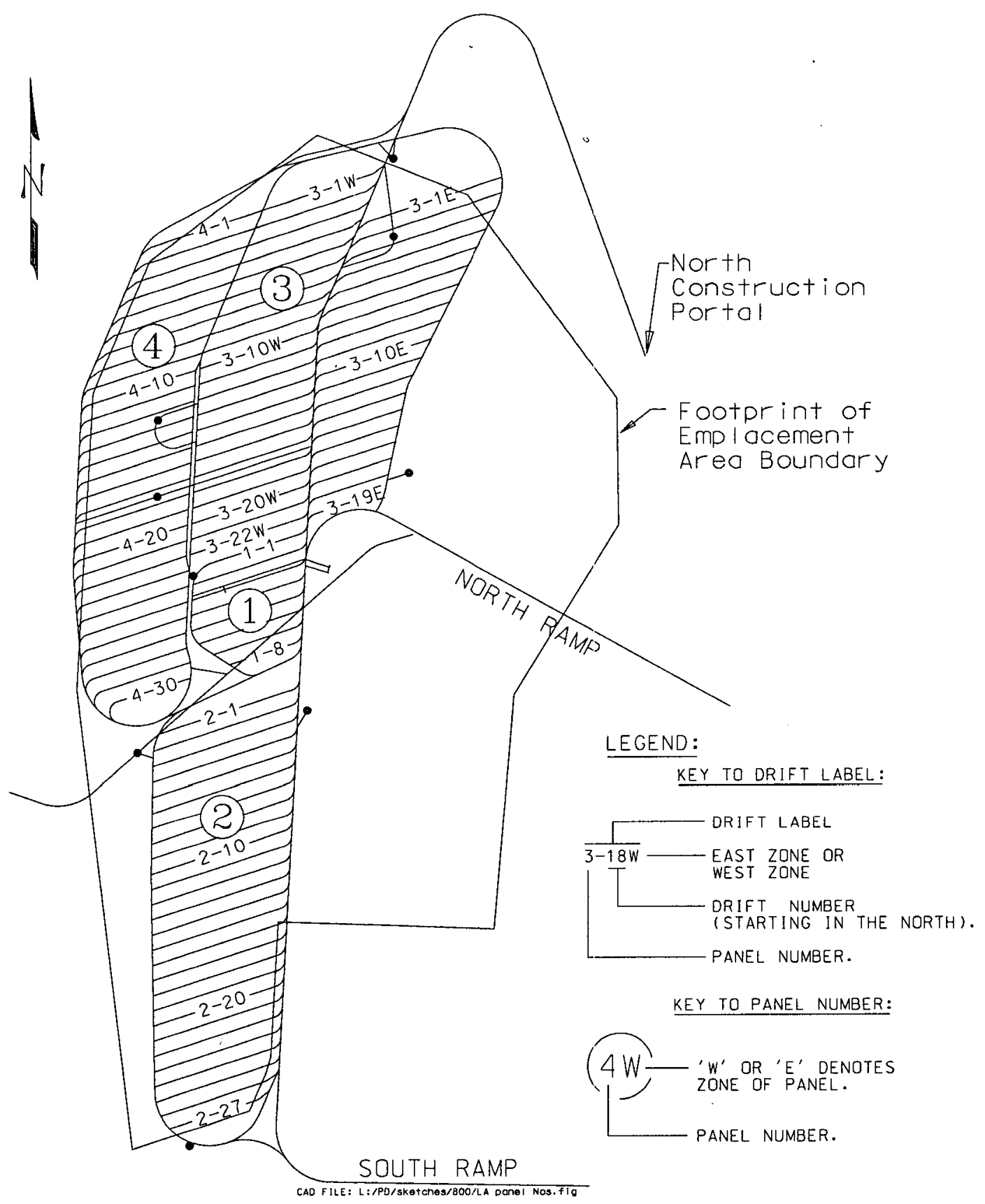

Figure 3. Underground Layout Configuration

\subsubsection{Shielding}

Shielding calculations determine radiation dose rates on waste package surfaces and in the vicinity of these surfaces. Radiation dose rate is a function of the radiation type, radiation energy spectrum and intensity, radiation interaction information, material compositions and densities, system geometry, and flux-to-dose rate conversion factors. In addition, radiation dose rate is dependent on the assumed location of the detector. 


\subsubsection{Radiation Source Terms}

The radiation source terms of the waste forms are an important input to shielding calculations. The radiation source terms consist of neutron and photon intensities as functions of their energies. The radiation source terms for commercial SNF have been generated for PWR and BWR fuel designs. These are documented in the following:

- PWR Source Term Generation and Evaluation (BSC 2004 [DIRS 169061)

- BWR Source Term Generation and Evaluation (BSC 2003 [DIRS 164364]).

For HLW glass, photon and neutron source terms are generated using the design basis glass developed at the Savannah River Site Defense Waste Processing Facility and are provided in Source Terms for HLW Glass Canisters (CRWMS M\&O 2000 [DIRS 151947], Attachment V, p. V-1, and Attachment VI, p. VI-1). The Savannah River Site source terms are used in shielding evaluations because they are expected to be the highest of all HLW glass. If future changes result in a higher level radioactive waste glass canister, it will be evaluated.

DOE SNF has been categorized into nine fuel groups:

1. Uranium Metal Fuels (N-Reactor fuel)

2. Uranium-Zirconium/Uranium-Molybdenum Fuels (Fermi Liquid Metal Reactor fuel)

3. Uranium Oxide Fuels (high enriched uranium fuels-Shippingport PWR fuel)

4. Uranium Oxide Fuels (low enriched uranium fuels-Three Mile Island-2 PWR fuel)

5. Uranium-Aluminum Fuels (foreign research reactor fuel)

6. Uranium/Thorium/Plutonium Carbide Fuels (Ft. St. Vrain Gas Cooled Reactor fuel)

7. Mixed Oxide Fuels (Fast Flux Test Facility Reactor fuel)

8. Uranium/Thorium Oxide Fuels (Shippingport Light Water Breeder Reactor fuel)

9. Uranium-Zirconium-Hydride Fuels (TRIGA fuel).

A representative fuel type is chosen as a bounding case for each group. Burnup, fissile enrichments, and total fuel mass determine the selection of the representative fuel used for shielding and criticality analysis. The bounding source terms for each fuel group are provided in the following documents.

- Shippingport LWBR (Th/U Oxide) Fuel Characteristics for Disposal Criticality Analysis (DOE 1999 [DIRS 105007])

- FFTF (MOX) Fuel Characteristics for Disposal Criticality Analysis (INEEL 2002 [DIRS 158820])

- Fermi (U-Mo) Fuel Characteristics for Disposal Criticality Analysis (DOE 1999 [DIRS 104110])

- Shippingport PWR (HEU Oxide) Fuel Characteristics for Disposal Criticality Analysis (DOE 1999 [DIRS 104940]) 
- TRIGA (UZrH) Fuel Characteristics for Disposal Criticality Analysis (DOE 1999 [DIRS 103891])

- $\quad$ Reactor (U-metal) Fuel Characteristics for Disposal Criticality Analysis (DOE 2000 [DIRS 150095])

- Fort Saint Vrain HTGR (Th/U Carbide) Fuel Characteristics for Disposal Criticality Analysis (Taylor 2001 [DIRS 154726])

- Statement of Work for DOE-Office of Civilian Radioactive Waste Management, Technical Assistance on Melt-Dilute Criticality and Shielding Analyses, Revision 2, May 30, 2001 (BSC 2001 [DIRS 155245])

- Intact and Degraded Mode Criticality Calculations for the Codisposal of TMI-2 Spent Nuclear Fuel in a Waste Package (BSC 2004 [DIRS 168935 ]).

\subsubsection{Radiation Interaction Information}

Radiation interaction information is available in the computer code packages used in shielding calculations (Sections 6.4.1.3 and 6.4.1.4). This information is derived from evaluated nuclear data libraries by processing it into formats suitable for the individual codes. An evaluated nuclear data library is produced by combining experimentally measured cross sections with results predicted by theoretical nuclear calculations, in an attempt to extract the most accurate cross-section information.

\subsubsection{Material Chemical Compositions and Densities}

Radiation interaction information contains microscopic cross sections of individual nuclides. A microscopic cross section may be interpreted as the effective cross-sectional area presented by the target atom or electron to the incident particle for a given interaction. Microscopic cross sections deal with probabilities of the interaction of radiation with individual targets (nuclei or electrons). Macroscopic cross sections (number density times microscopic cross section) are related to probabilities for interaction with the aggregate of targets that compose the medium through which the radiation is passing. Macroscopic cross sections have units of reciprocal length $\left(\mathrm{cm}^{-1}\right)$, and act as linear attenuation coefficients in photon transport. Shielding analyses require macroscopic cross sections in order to determine interactions of radiation with the materials of the system. The codes calculate the macroscopic cross sections based on material compositions and densities, and, in the case of Monte Carlo radiation transport codes, the macroscopic cross sections of the materials determine the collision site and select the target element. The chemical compositions and densities of the materials in a waste package shielding calculation are available in the American Society for Testing and Materials standards database.

\subsubsection{Flux-to-Dose Rate Conversion Factors}

Flux-to-dose rate conversion factors are contained in the computer code packages used in shielding calculations (Sections 6.4.1.3 and 6.4.1.4). 


\subsubsection{Criticality Analyses}

Criticality analyses are performed to assure waste forms will not be loaded in a manner leading to the formation of a critical mass and, consequently, a nuclear criticality event. The process for analyzing the potential for criticality during the preclosure period is described in Preclosure Criticality Analysis Process Report (BSC 2004 [DIRS 169590]). A risk-informed methodology will be used for criticality analyses during the postclosure period. Details of the criticality analysis models, including inputs, are given in the following reports: Errata for Isotopic Model Report for Commercial SNF Burnup Credit (BSC 2003 [DIRS 168037]), Configuration Generator Model for In-Package Criticality (BSC 2003 [DIRS 165629]), Geochemistry Model Validation Report: External Accumulation Model (BSC 2001 [DIRS 156324]), Geochemistry Model Validation Report: Material Degradation and Release Model (BSC 2001 [DIRS 156790]), and Criticality Model Report (BSC 2003 [DIRS 165733]).

\subsection{INTERFACE WITH OTHER PROJECT ORGANIZATIONS}

Waste package, emplacement pallet, and drip shield design input is dependent on several parallel tasks including facility design, preclosure safety analysis, and fabrication methods. Interface Exchange Drawings define the interfaces with the Performance Assessment Project.

Facilities design is in an early stage at the present time and will not be complete at the time of License Application submittal. Additional design analysis will be performed, if necessary, as the facilities design matures and risks are evaluated.

\section{WASTE PACKAGE COMPONENT DESIGN ASSUMPTIONS}

The assumptions involved in making calculations or performing analytical work may be divided into two categories. The first category consists of generic assumptions that can be part of a calculation process or computational tool. Appropriate generic assumptions are addressed in this report that relate to the processing and disposal of the range of waste forms in the repository.

The second category of assumptions are specific assumptions related to a particular analysis or calculation. An example of a specific assumption is an assumption necessary to perform a drop calculation for a particular waste package. Some, but not all, specific assumptions are addressed in this report. Each individual calculation report describes all assumptions pertaining to the specific calculation.

None of the assumptions listed in this section were used in this report to produce quality related results in the sense of generating numeric values or defining a specific component design; however, they define the assumptions appropriate to the methodology. If the methodology is used in consonance with this document to produce quality related results in future analyses, then the quality of these assumptions (and whether or not they require further verification) will be determined at that time.

\subsection{SOURCE TERM GENERATION ASSUMPTIONS}

Source term calculations provide heat generation rates, photon and neutron spectra and intensities, and radionuclide inventories of commercial SNF assemblies, DOE SNF, and HLW. 
The heat generation rates are used in thermal evaluations of the waste packages and the host rock of the repository. The photon and neutron sources are used to determine the radiation level surrounding a waste package. The radionuclide inventories are used to determine dose rates due to the release of radionuclides from the waste packages during the postclosure period. The source terms discussed in this section are not used for criticality. Source term calculations for criticality are discussed in Sections 4.1.4.4 and 5.5.

\subsubsection{Commercial Spent Nuclear Fuel Waste Forms}

Assumptions related to the evaluation of source terms from commercial SNF waste forms are discussed in the following sections.

\subsubsection{Generic Fuel Assemblies and Burnup Histories}

It is assumed that the commercial SNF waste stream can be approximated by calculating source terms for generic PWR and BWR assemblies at incremental enrichments and burnups and that a generic burnup history (without the modeling of outages, intermittent down times, etc.) can be used for the depletion calculations. The rationale for this assumption is based on the analysis provided in NUREG/CR-5625 (Hermann et al. 1994 [DIRS 154045]), which shows that the heat generation rates of generic PWR and BWR SNF assemblies, in watts per $\mathrm{kg} \mathrm{U}$, do not vary significantly with details of irradiation history for a given burnup and cooling time between 5 and 100 years. Design basis values of fuel parameters (enrichment, burnup, and aging) can be different for shielding analysis and thermal analysis.

The decay heat rates include the contributions from the radiation generated by the fission products and from the radiation generated by the actinides in commercial SNF. The fission product contribution dominates the decay heat generation initially, but its importance decreases with time relative to the actinide contribution. Fission product generation is sensitive to the assembly burnup, which is determined by the total number of fissions. It is less sensitive to the neutron spectra or actinide compositions because the fission yields vary slowly with these variables. While the irradiation history, especially for the last reactor cycle, will greatly influence the short-lived fission products, the dependence of the decay heat rate on the specific power exists only for the first five years of cooling. The mandatory five-year cooling period before waste acceptance, as dictated in $10 \mathrm{CFR} 961.11$, will progressively decrease the dependence of the heat generation rate on the short-lived fission products and allow for the use of generic burnup histories for the depletion calculations. This assumption is used in Section 6.1.

\subsubsection{Uniform Specific Power}

An average uniform specific power over the entire length of the assembly is assumed, and the total irradiation interval is determined as the ratio of the assembly burnup to the specific power. The rationale for using a uniform power within the assembly volume is that the subsequent shielding and thermal evaluations can conservatively take into account the burnup axial profile through an axial peaking factor (Section 5.4.1). This assumption is used in Section 6.1. 


\subsubsection{Interpolation in Arrays of Results}

It is assumed that the source terms can be generated for an array of various enrichments, burnups, and decay times and that interpolation can then be used to obtain the source terms of any specific assemblies in the waste stream without requiring explicit modeling of the assemblies. The rationale for this assumption is that, as described in Section 5.1.1.1, for a given burnup and cooling time during the repository preclosure period, the decay heat rate, in watts per $\mathrm{kg} \mathrm{U}$, is relatively constant for different fuel assembly types. For a sufficient number of enrichments, burnups, and decay times, the source term error resulting from interpolation is on the order of the resolution of the calculational methods. This assumption is used in Section 6.1.

\subsubsection{Assembly Mass Loading}

As discussed in section 4.2.1.1, the B\&W Mark B assembly is used as the generic PWR fuel lattice for analysis due to its high initial heavy metal loading and large amount of stainless steel and Inconel hardware. It is assumed that the initial heavy metal loading of a PWR assembly is $475 \mathrm{~kg}$ of heavy metal, instead of the $464 \mathrm{~kg}$ of a typical B\&W Mark B assembly (Punatar 2001 [DIRS 155635], Table 3.1). For a BWR assembly, the initial heavy metal loading is assumed to be $200 \mathrm{~kg}$, instead of the $184 \mathrm{~kg}$ of a typical GE $8 \times 8 \mathrm{BWR}$ fuel assembly (Larsen et al. 1976 [DIRS 146576], p. A-2). The rationale for these assumptions is that a higher initial uranium loading leads to a proportionally higher source term, which is generally more conservative for design considerations. Design basis values of fuel parameters (enrichment, burnup, and aging) can be different for shielding analysis and thermal analysis. This assumption is used in Section 6.1.

\subsubsection{Non-Commercial Spent Nuclear Fuel Waste Forms}

Assumptions related to evaluation of source terms from non-commercial SNF waste forms are discussed in the following sections.

\subsubsection{U.S. Department of Energy Spent Nuclear Fuel Waste Forms}

For DOE SNF, it is assumed that the total initial radionuclide inventory provided by Idaho National Engineering and Environmental Laboratory (DOE 2003 [DIRS 163377]) is adequate for the analyses of the repository at Yucca Mountain. The rationale for this assumption is that the Idaho National Engineering and Environmental Laboratory has generated inventories for several representative fuel types in the DOE SNF waste stream, which are used to generate radionuclide inventories for the rest of the waste stream. This assumption is used in Section 6.1.2.2.

\subsubsection{Defense High-Level Radioactive Waste}

For HLW in borosilicate glass logs, the defense HLW historical information regarding the inventory at the various sites is used in decay calculations to generate initial radionuclide inventories (originally DOE 2002 [DIRS 155970], Appendix A, and later Hamel 2003 [DIRS 164947], Allison 2004 [DIRS 168734], and WVNS 2003 [DIRS 168661])). These inventories are then used in ORIGEN-S decay calculations to obtain source terms over time. The ORIGEN$S$ code is described in Section 6.1.1.2. It is assumed that the information for the material planned for disposal at Yucca Mountain is adequately represented by the radionuclide inventories 
provided by the sites. Again, the rationale for this assumption is that the information provided represents an average of the material, not the bounding. This assumption is used in Section 6.1.2.3.

\subsection{STRUCTURAL ANALYSIS ASSUMPTIONS}

A wide array of structural calculations assumptions is discussed in the following sections.

\subsubsection{Contact Stiffness Between Waste Package and Impact Surface}

For ANSYS calculations, the assumption is made that the contact stiffness between the waste package and the impact surface can be determined iteratively. The rationale for this assumption is explained in the following paragraph. LS-DYNA calculations do not require this assumption.

The magnitude of the contact stiffness (between surfaces used in the simulation) is a parameter that influences the resulting stresses. If the stiffness value is very large; stiffness matrix ill-conditioning and divergence will occur. Similarly, an extremely small stiffness value results in compatibility violations. Therefore, an optimum value for the contact stiffness is one that is between the two and is arrived at iteratively. Therefore, an iterative process to determine the value of contact stiffness used in the finite element simulation is deemed acceptable for transient dynamic solutions. This assumption is used in Section 6.2.2.2.

\subsubsection{Emplacement Pallet Lift}

The acceleration used for the emplacement pallet lift is $1.0 \mathrm{~m} / \mathrm{s}^{2}$ (total upward acceleration being $10.81 \mathrm{~m} / \mathrm{s}^{2}$ including gravity). The rationale for this assumption is that it conservatively represents the lifting of the emplacement pallet by the emplacement gantry and provides a basis for the lifting strength of the emplacement pallet. This assumption is used in Section 6.2.2.2.

\subsubsection{Geometry of Collapsed Drift}

The deterioration of the rock blocks surrounding the potential repository emplacement drifts increases the external pressure on the drip shield. This additional loose rock load, which takes place in lithophysal units, will be taken into structural design considerations in accordance with the information provided in Drift Degradation Analysis (BSC 2004 [DIRS 168550], Section 6.4). This assumption is used in Section 6.2.2.2.

\subsubsection{First Failure of Inner Vessel Lid for Pressurization Event Sequence}

For evaluation of the pressurization event sequence, the inner vessel lid is assumed to fail before the outer lid; however, no structural credit is assumed for the outer lid. The rationale for this assumption is that, despite the ductile nature of the inner vessel, the gap between the inner vessel lid and outer lid at the waste package bottom end is large enough that the inner vessel lid can experience a nonlinear deformation sufficient to develop a through-wall crack. This assumption is used in Section 6.2.2.3. 


\subsubsection{Strain Rate Effect on Material Properties}

Strain rate effects are also known as material strain-rate sensitivity or viscoplasticity. Of all materials that are currently used in engineered barrier system, the strain rate data is only available for 304 stainless steel and is used when possible. Where strain rate data is not available, the material properties obtained under the static loading conditions are used. The impact of using material properties obtained under static loading conditions is small. The rationale for this assumption is that the change of mechanical properties of subject materials at the peak strain rates that typically occur during the simulated events does not have significant effect on the results presented in calculations. This assumption is used in Sections 6.2.2.4 and 6.2.2.5.

\subsubsection{Uniform Strains}

\subsubsection{Uniform Strain of SA-516 K02700 Carbon Steel}

The uniform strain (the strain corresponding to the uniaxial tensile strength) of SA-516 K02700 carbon steel is not available in literature. Therefore, it is conservatively assumed that the uniform strain is 50 percent of the elongation. The rationale for this assumption is the character of the stress-strain curve for SA-36 carbon steel (Boyer 2000 [DIRS 152656], p. 305; Bowles 1980 [DIRS 153409], Figure 1-3, p. 11), which has a similar chemical composition as SA-516 K02700 carbon steel (ASME 2001 [DIRS 158115], Section II, Part A, SA-516/SA-516M, Table 1 and SA-36/SA-36M, Table 2). This assumption is used in Section 6.2.2.5.

\subsubsection{Uniform Strain of 316L Stainless Steel}

The uniform strain of $316 \mathrm{~L}$ stainless steel is not available in the open literature. Therefore, it is conservatively assumed that the uniform strain is 60 percent of the elongation. The rationale for this assumption is the character of the stress-strain curve for 316L stainless steel (Boyer 2000 [DIRS 152656], p. 305). This assumption is used in Section 6.2.2.6.

\subsubsection{Uniform Strain of SB-575 N06022 (Alloy 22 N06022) and SA-240 S31600 (SS 316)}

Three-stage deformation characteristics are not observed in the stress-strain curves for Alloy 22 N06022 (DTN: LL020603612251.015 [DIRS 160430]) or Type 316 (Boyer 2000 [DIRS 152656], p. 302) stainless steel. Therefore, it is conservatively assumed that the uniform strain is 90 percent of the elongation. The rationale for this assumption is the character of the stressstrain curves for Alloy 22 N06022 and Type 316 stainless steel. This assumption is used in Section 6.2.2.5.

\subsubsection{Uniform Strain of 304 Stainless Steel}

The uniform strain of 304 stainless steel is not available in literature. Therefore, it is conservatively assumed that the uniform strain is 75 percent of the elongation. The rationale for this assumption is the character of stress-strain curve for 304 stainless steel (Boyer 2000 [DIRS 152656], p. 294). This assumption is used in Section 6.2.2.5. 


\subsubsection{Uniform Strain of Ti-7 and Ti-24}

The uniform strains of Ti-7 and Ti-24 are not available in literature. Therefore, it is assumed that the uniform strain is equal to the elongation. The rationale for this assumption is that a small change in tangent modulus does not significantly affect the results of this calculation. This assumption is used in Sections 6.2.2.4 and 6.2.2.6.

\subsubsection{Assumptions for Missile Impact Analysis}

Assumptions for structural calculations of missile impact are discussed in this section. The analysis is for missiles resulting from Category 1 or Category 2 event sequences and not for missiles due to terrorism.

\subsubsection{Application of Empirical Relationships for Impact Analysis}

Empirical equations were developed for the perforation of ductile mild steel plates (Jones 1994 [DIRS 137700]). These relations are assumed to give conservative results for the waste package inner vessel and outer corrosion barrier. The rationale for this assumption is that nickel alloys have higher strength and ductility than mild steels (low-carbon steels), and the ductility of stainless steel is higher than that of mild steel. Therefore, these empirical relations can be conservatively used to approximate behavior of the waste package inner vessel and outer corrosion barrier impacted by an accidentally released missile. This assumption is used in Section 6.2.2.4.

\subsubsection{Assumption of Waste Package as Flat Plate for Impact Analysis}

Empirical relations of flat plates are assumed to be applicable to determining the structural response of the waste package to an impact load. The fixed-end boundary conditions of the waste package in the localized region of impact are assumed to be similar to those of the flat plate shown in the reference (Jones 1994 [DIRS 137700], Figure 1, p. 57). The rationale for this assumption is that the effect of shell curvature is small compared to the plate thickness and the waste package in the vicinity of the impact region will resemble a geometric configuration similar to a flat plate with fixed ends (Figure 4). This assumption is used in Section 6.2.2.4.

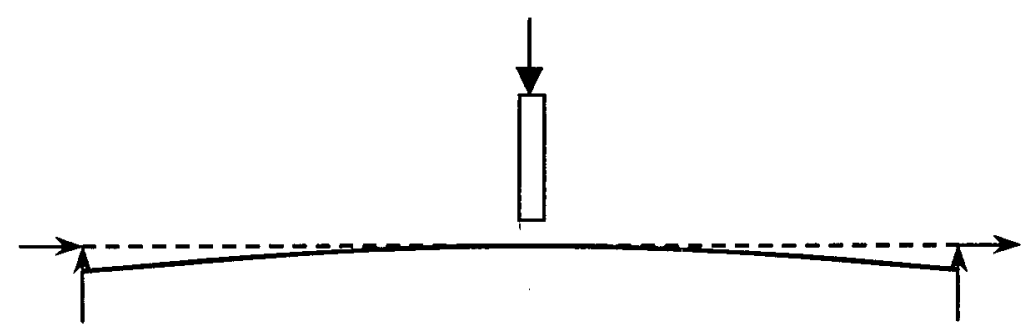

Figure 4. Flat Plate Representation of Impact Analysis

\subsubsection{General Assumptions for Waste Package Drop Analyses}

The following assumptions are used for the waste package tipover, swing down, corner drop, horizontal drop, and vertical drop analyses. As the preliminary and final hazards analyses are 
completed, the drop analyses will be reviewed to ensure that design parameters are consistent with the hazards identified. Assumptions specific to each drop analysis are presented in separate subsections.

The drops and drop heights, taken from Preliminary Nuclear Safety Design Bases for License Application (BSC 2003 [DIRS 165182] Table A-2, Page A-13), are defined as:

- Waste Package Tipover - the waste package is at rest on the ground in a vertical position and an external force (such as a seismic event) causes the waste package to tip over and impact the ground. A tipover from an elevated surface is also considered and bounds the tip over from a flat surface due to the higher impact energy.

- Waste Package Swing Down - the waste package is being lifted in a horizontal orientation at a height of $2.4 \mathrm{~m}$ when the lifting device inadvertently releases one end. One end of the waste package remains held by the lifting device while the other end swings down and impacts the ground.

- Waste Package Corner Drop-the waste package is being lifted in a vertical orientation at a height of $2.0 \mathrm{~m}$ when the lifting device inadvertently drops it. A corner of the waste package impacts the ground first.

- Waste Package Horizontal Drop-the waste package is being lifted in a horizontal orientation at a height of $2.4 \mathrm{~m}$ when the lifting device inadvertently drops it. The waste package impacts the ground squarely on its side.

- Waste Package Vertical Drop-the waste package is being lifted in a vertical orientation at a height of $2.0 \mathrm{~m}$ when the lifting device inadvertently drops it. The waste package impacts the ground squarely on its base.

- Horizontal Drop with Emplacement Pallet--the emplacement pallet with waste package is being lifted in a horizontal orientation when the lifting device inadvertently drops it from a height of $2.0 \mathrm{~m}$ (floor to pallet, or $2.4 \mathrm{~m}$ floor to waste package). The emplacement pallet with waste package impacts the ground along its horizontal axis. This is also done as a horizontal drop onto the emplacement pallet. The emplacement pallet is the object considered that may puncture the waste package.

The drop analyses are performed with the trunnion collar attached to the fully loaded waste package because that is the normal condition for moving the waste package. The swing down and corner drops are variations of the vertical and horizontal drops and use the same drop heights listed for the vertical and horizontal drops. These assumptions are used in Section 6.2.2.5.

\subsubsection{Unyielding Target Surface}

The target surface for the falling or dropped waste package is assumed to be unyielding. The rationale for this assumption is that it maximizes the stress on the falling waste package. This assumption is used in Section 6.2.2.5. 


\subsubsection{Poisson's Ratio for Alloy 22 N06022}

Poisson's ratio of Alloy 22 N06022 is not available in the literature. The Poisson's ratio of Alloy 625 (SB-443 N06625) is assumed for Alloy 22 N06022 because the chemical compositions of Alloy 22 N06022 (ASME 2001 [DIRS 158115], Section II, Part B, SB-575, Table 1) and Alloy 625 (ASM 1980 [DIRS 104317], p. 143) are similar. Hence, the impact of this assumption is negligible. This assumption is used in Section 6.2.2.5.

\subsubsection{Poisson's Ratio for SA-516 Carbon Steel}

Poisson's ratio is not available for SA-516 carbon steel. Therefore, Poisson's ratio of cast carbon steel is assumed for SA-516 carbon steel. The rationale for this assumption is that the elastic constants of cast carbon steels are only slightly affected by changes in composition and structure (ASM 1978 [DIRS 102018], p. 393). Hence, the impact of this assumption is minimal. This assumption is used in Section 6.2.2.5.

\subsubsection{Poisson's Ratio of 316L Stainless Steel}

The Poisson's ratio of 316L stainless steel is not available in the literature. The Poisson's ratio of 316 stainless steel (SA-240 S31600) is assumed for 316L stainless steel. The rationale for this assumption is the similar chemical compositions of these two stainless steels (ASME 2001 [DIRS 158115], Section II, Part A, SA-240, Table 1). Hence, the impact of this assumption is minimal. This assumption is used in Section 6.2.2.6.

\subsubsection{Poisson's Ratio for 304L Stainless Steel}

Poisson's ratio for 304L stainless steel is not available in the literature. Therefore, it is assumed to be the same as Poisson's ratio for 304 stainless steel. The rationale for this assumption is that the chemical compositions of 304L stainless steel and 304 stainless steel are similar (ASME 2001 [DIRS 158115], Section II, Part A, SA-240, Table 1). Hence, the impact of this assumption is minimal. This assumption is used in Section 6.2.2.6.

\subsubsection{Temperature-Dependent Material Properties}

Some of the temperature-dependent material properties (density, Poisson's ratio, and elongation) are not available for SB-575 N06022 (Alloy 22 N06022), SA-705 S017400 (17-4 PH), SB-265 R52400 (Titanium Grade 7), SB-265 R56400 (Titanium Grade 24), SA-516 K02700 (SA-516 carbon steel), SA-240 S31600 (316 stainless steel), SA-240 S31603 (316L stainless steel), SA-240 S30403 (304L stainless steel), and SA-240 S30400 (304 stainless steel) (Poisson's ratio only). The room temperature $\left(20^{\circ} \mathrm{C}\right)$ material properties are assumed for these materials. The impact of using room-temperature material properties is anticipated to be small. The rationale for this assumption is that undetermined mechanical properties of said materials will not significantly impact the results. This assumption is used in Section 6.2.2.5.

\subsubsection{Material Elongation Properties}

The elongation properties of Alloy 22 N06022 and 316 stainless steel at elevated temperatures is not available from codes and standards. However, vendor data is available for the typical 
elongation of Alloy 22 N06022 and 316 stainless steel (Haynes International 1997 [DIRS 100896], p. 15; Allegheny Ludlum 1999 [DIRS 151409], p. 8, respectively). For comparison purposes, the percent difference between typical elongation at room temperature and elevated temperatures can be normalized and applied to the data available from accepted codes, thus ensuring that the bounding case is covered. The rationale for this assumption is to be as reasonably accurate as possible. It would not be logical for the elongation values to remain constant over the range of temperatures under consideration. This assumption is used in Section 6.2.2.5.

\subsubsection{Poisson's Ratio for 17-4 PH SS in the H900 Condition}

The Poisson's Ratio for 17-4 PH in the H900 condition is not available from traditional sources. It is assumed that this value is 0.272 . The rationale for this assumption is that this is what is available from vendor resources (High Temp Metals 2002 [DIRS 158006]). The impact of this assumption is anticipated to be negligible. This assumption is used in Section 6.2.2.6.

\subsubsection{Friction Coefficient between 316 Stainless Steel and 314L Stainless Steel}

The friction coefficient for contact between 316 stainless steel and $316 \mathrm{~L}$ stainless steel is not available in literature. It is, therefore, assumed that the dynamic (sliding) friction coefficient for this contact is 0.4 . The rationale for this assumption is that this friction coefficient represents the lower bound for the steel-on-steel contacts (Avallone and Baumeister 1987 [DIRS 103508], Table 3.2.1, p. 3-26; Meriam and Kraige 1987 [DIRS 104306], p. 441). This assumption is used in Section 6.2.2.6.

\subsubsection{Temperature Dependent Properties for Titanium Grade 24}

The temperature-dependent tensile and yield strength of Ti-24 are not available through the ASME Boiler and Pressure Vessel Code (ASME 2001 [DIRS 158115]). However, vendor data for these material properties are available (TIMET 2000 [DIRS 160688], Figure 1). Therefore, the tensile and yield strengths of Ti-24 from TIMET at elevated temperatures will be normalized and used with the room temperature values from ASME to calculate the tensile and yield strength of Ti-24 at elevated temperatures. The rationale for this assumption is that the ASME Boiler and Pressure Code states the minimum material properties. Therefore, calculating the minimum properties at elevated temperatures with this method provides a bounding value of tensile and yield strength of $\mathrm{Ti}-24$ for this calculation.

The temperature-dependent modulus of elasticity of Titanium Grade 24 is not available through the ASME Code or through the ASM Metals Handbook (ASM International 1990 [DIRS 141615]). However, vendor data are available for this material property (TIMET 2000 [DIRS 160688], Table 2). Therefore, the modulus of elasticity of Ti-24 from TIMET at elevated temperatures will be normalized and used with room temperature values from the ASM Metals Handbook (ASM International 1990 [DIRS 141615], Table 21, p. 621) to calculate the modulus of elasticity of Ti-24 at elevated temperatures. The rationale for this assumption is that the room temperature data is only adjusted by the trend in the vendor data and not by the absolute values in the vendor data. This assumption is used in Section 6.2.2.6. 


\subsubsection{Structural Properties for Neutronit A 978}

Structural properties including density, yield stress, ultimate stress, percent elongation, Young's Modulus, thermal expansion coefficient, and Poisson's ratio are not available for Neutronit A 978. Therefore, the corresponding properties for Neutronit A 976 are assumed for Neutronit A 978. The rationale for this assumption is that the two materials have similar chemical compositions. This assumption is used in Section 6.2.2.

\subsubsection{Assumptions for the Representation of Waste Package Loaded Internals}

The following assumptions may be used in applicable individual calculations.

\subsubsection{Commercial Waste Form Geometry}

The exact geometry of the waste form is simplified in such a way that its total mass is assumed to be distributed within a bar of square cross section with uniform mass density. The rationale for this assumption is to provide a simplified finite element representation without affecting computational results. This assumption is used in Section 6.2.2.6.

\subsubsection{Commercial Waste Form Material}

The waste form is assumed to be made of 304 stainless steel. The rationale for this assumption is that the end fittings of the form are made of 304 stainless steel (Punatar 2001 [DIRS 155635], Section 2.1, p. 2-4 [for PWR]; Stout and Leider 1997 [DIRS 100419], p. 2.1.2.3 [for BWR]), and these are the parts that will come in contact with other components. This assumption is used in Section 6.2.2.6.

\subsubsection{Removal of Thermal Shunts}

The thermal shunts are removed for the purpose of structural calculations. The rationale for this assumption is that the purpose of the thermal shunts is not to provide structural support. Their removal provides a bounding set of results, while simplifying the finite element representation. This assumption is used in Section 6.2.2.6.

\subsubsection{Defense High-Level Radioactive Waste Form and Internals}

The exact geometry of the loaded internals is simplified for the purpose of structural calculations. The waste package internal structures, borosilicate glass canisters, and all other internals are created as a solid cylinder with an appropriate mass value. The rationale for this assumption is that it simplifies the finite element representation and conservatively neglects energy absorption within the internal components. This assumption is used in Section 6.2.2.6.

\subsubsection{Pressurized Water Reactor Waste Form Design Parameters for 21-PWR}

The following design parameters are assumed for a PWR SNF assembly to be loaded into a 21PWR waste package: mass $=1705 \mathrm{lbs}(773.4 \mathrm{~kg})$, width $=8.5 \mathrm{in} .(216.9 \mathrm{~mm})$, and length $=174$ in. (4407 mm). The rationale for this assumption is that these parameters correspond to the B\&W $15 \times 15$ fuel assembly, which is the heaviest PWR fuel assembly available (BSC 2003 
[DIRS 167273], Table 4). The mass of the B\&W $15 \times 15$ fuel assembly has been increased by $25 \mathrm{lbs}(11.4 \mathrm{~kg})$ to account for variations in fuel assembly mass. This assumption is used in Section 6.2.2.

South Texas PWR fuel assemblies will not be disposed in the 21-PWR waste package and are therefore excluded from this assumption. Design parameters for South Texas assemblies will be established during future design work for the 12-PWR waste package.

\subsubsection{Assumed Geometry of Lid Lifting Features for Waste Package Tip-Over}

The geometry of the lid lifting features is simplified for tip-over calculations. The total mass of each lifting feature is assumed to be distributed within a disc with uniform mass density and constructed of the same material as the lid to which it is attached. The rationale for this assumption is that only the mass of the lifting feature has an effect on structural calculations. This assumption is used in Section 6.2.2.5.

\subsubsection{Assumed Angular Velocity for Waste Package Swing Down Analysis}

For the purposes of analyzing the initial angular velocity of the waste package before impact, the waste package will be assumed to be a solid cylinder. This is necessary to calculate the rotary moment of inertia. The impact of this assumption on the results is negligible. The rationale for this assumption is the overall cylindrical shape of the waste package and the relatively solid packing of the contents. This assumption is used in Section 6.2.2.5.

\subsubsection{DHLW/DOE-Short Waste Package Swing Down Height}

The angle of impact for the $5 \mathrm{DHLW} / \mathrm{DOE}-$ Short waste package swing down is assumed to be 45 degrees. A swing down event is usually calculated as starting from the maximum allowable horizontal lift height of $7.9 \mathrm{ft}(2.4 \mathrm{~m})$; however, in this case the waste package would not reach the ground if swung down from that height. The rationale for this assumption is that 45 degrees will provide a good representation of a swing down event for this waste package. This assumption is used in Section 6.2.2.5.

\subsubsection{Assumed Geometrical Arrangement of Waste Package for Corner Drop Analysis}

For corner drop analysis, the waste package is assumed to strike the ground at an angle that puts the center of mass, which is assumed to coincide with the geometric center of the waste package, directly above the point of impact. The rationale for this assumption is that it induces the highest impact load on the waste package outer corrosion barrier. This assumption is used in Section 6.2.2.5.

\subsubsection{Additional Assumptions for Waste Package Vertical Drop Analysis}

Assumptions for structural calculations of vertical drop analysis are discussed in this section. 


\subsubsection{Friction Coefficients for Vertical Drop Contacts Involving Alloy 22 N06022}

The friction coefficients for contacts involving Alloy 22 N06022 are not available in literature. It is, therefore, assumed that the dynamic (sliding) friction coefficient for all contacts is 0.4 . The rationale for this assumption is that this friction coefficient represents the lower bound for most dry contacts involving steel and nickel (Meriam and Kraige 1987 [DIRS 104306], p. 441; Avallone and Baumeister 1987 [DIRS 103508], Table 3.2.1, p. 3-26), nickel being the dominant component in Alloy 22 N06022 (ASME 2001 [DIRS 158115], Section II, Part B, SB-575, Table 1). This assumption is used in Section 6.2.2.5.

\subsubsection{Variation of Functional Friction Coefficient:}

The variation of functional friction coefficient between the static and dynamic values as a function of relative velocity between the contact surfaces is not available in literature for the materials used in this calculation. Therefore, the effect of relative velocity of the surfaces in contact is neglected in these calculations by assuming that the functional friction coefficient and static friction coefficient are both equal to the dynamic friction coefficient. The impact of this assumption is anticipated to be negligible. The rationale for this conservative assumption is that it provides the bounding set of results by minimizing the friction coefficient within the given finite element analysis framework. This assumption is used in Section 6.2.2.5.

\subsubsection{Additional Assumptions for Waste Package Puncture Drop Analysis}

Drops onto objects that might puncture a waste package are not expected to occur. If future evaluations are needed, additional assumptions for structural calculations of waste package puncture drop analysis are described in the following sections.

\subsubsection{Contact Stiffness Between the Waste Package and the Emplacement Pallet}

The magnitudes of the contact stiffness between (1) the waste package and the emplacement pallet and (2) the emplacement pallet and unyielding surface is assumed to be $1 \times 10^{9} \mathrm{~N} / \mathrm{m}$. The rationale for this assumption is that this magnitude of contact stiffness between surfaces results in simulation convergence and provides results that satisfy compatibility requirements. If the contact stiffness value is too large, stiffness matrix ill-conditioning and divergence occur. On the other hand, a contact stiffness that is too small will result in compatibility violations. Therefore, an optimum value for the contact stiffness is one that is in between the extremes and is derived by iteration. The iterative process is based on engineering judgment. It should be noted that the contact stiffness is neither a measurable nor an intrinsic property of the materials in contact. This assumption is used in Section 6.2.2.6.

\subsubsection{Bending Stiffness of the Inner Vessel and Outer Corrosion Barrier}

The bending stiffness (plate constant) of the inner vessel and outer corrosion barrier is calculated by assuming that the expression for the bending stiffness of a flat plate is also valid for the waste package. The rationale for this assumption is that the cliameter of the waste package is much larger than the respective thickness. This assumption is used in Section 6.2.2.6. 


\subsubsection{Assumptions for Rockfall Upon Waste Package and Drip Shield}

Additional assumptions for structural calculations of rockfall analysis for waste package and drip shield are described in the following sections.

\subsubsection{Material Properties for TSw2 Rock}

Temperature-dependent material properties are not available for TSw2 (Topopah Spring WeldedLithophysal Poor) rock. Hence, room temperature material properties are assumed for this material. The impact of using constant material properties is anticipated to be small. The rationale for this assumption is that the material properties of the rock do not have dominant impact on the calculation results. The likely exception is the yield strength of the rock, which decreases with the increasing temperature. Thus, the representation of the rock as an elasticideally-plastic solid with room temperature yield strength is conservative.

\subsubsection{Compressive Strength of TSw2 Rock}

The unconfined compressive strength of the TSw2 is available (DTN: MO0003RIB00079.000 [DIRS 148295], Table 1). The scatter of data is large, ranging from $31.6 \mathrm{MPa}$ to $288.9 \mathrm{MPa}$, as expected for rocks and brittle materials in general. For the purpose of the rockfall calculations, the unconfined compressive strength of the TSw2 is assumed to be $70 \mathrm{MPa}$. The rationale for this assumption is that it leads to conservatively bounding set of results in terms of stresses and strains in the outer corrosion barrier of the waste package.

\subsubsection{Young's Modulus and Poisson's Ratio of TSw2 Rock}

The modulus of elasticity and Poisson's ratio of the TSw2 are characterized by significant scatter of data (DTN: MO0003RIB00079.000 [DIRS 148295], Tables 3 and 4, respectively). For the purpose of rockfall calculations, modulus of elasticity is assumed to be $33 \mathrm{GPa}$, and Poisson's ratio 0.21 . The rationale for this assumption is that these values agree well with typical values of said properties for most rocks of interest (DTN: MO0003RIB00079.000 [DIRS 148295], Tables 3 and 4).

\subsubsection{Density of TSw2 Rock}

The density of the TSw 2 is assumed to be $2370 \mathrm{~kg} / \mathrm{m}^{3}$. The rationale for this assumption is that this value agrees well with all Topopah Spring Welded rocks and is not exceeded by any of other rock in the area (DTN: MO9808RIB00041.000 [DIRS 104850], Table 5). This assumption has no effect on the calculation results because the important input parameter is rock mass, regardless of the density.

\subsubsection{Friction Coefficients for Contacts Involving Seismic Rockfall Analysis}

The friction coefficient for contacts occurring between the rock and Ti-7 or invert and Alloy 22 N06022 is not available in literature. It is, therefore, assumed that the dynamic (sliding) friction coefficient for this contact is $\mathbf{0 . 5}$. The rationale for this assumption is that this friction coefficient represents a reasonable estimate based on available information for metal-on-stone contacts (Beer and Johnston 1977 [DIRS 145138], Table 8.1, p. 306). 


\subsubsection{Rock Geometry for Rockfall Analysis}

The rock shape is assumed to be rectangular prism. The rationale for this assumption is: the rock block data shows that some of the rock blocks are essentially rectangular prism. A finite element representation of the rock with an inclined rectangular prism provides a conservative approach from the point of view that the rock center of gravity is located directly above the point of impact. Hence, transferring the maximum linear momentum to the drip shield. The sharp edge of the prism also results in maximum strain on the drip shield plate. Vertex coordinates of the prism are obtained from "Results from 3DEC Nonlithophysal Rockfall Analyses with 10-7 Ground Motion Level” (DTN: MO0301MWD3DE27.003 [DIRS 161536]) and used to calculate the enveloping dimensions. This assumption is used in Section 6.2.2.4.

\subsubsection{Material Properties for Titanium}

Temperature-dependent Poisson's ratio is not available for Ti-7 (titanium Grade 7) and Ti-24 (titanium Grade 24). Therefore, the room temperature: Poisson's ratio is assumed for these materials. The impact of using Poisson's ratio at room temperature is anticipated to be small. The rationale for this assumption is twofold: first, for the subject materials, this property does not change significantly at the temperature of interest in this calculation; secondly, the material property in question does not have dominant impact on the calculation results. This assumption is used in Section 6.2.2.4.

The rate-dependent material properties obtained under the static loading conditions are used for dynamic loading of Ti-7 and Ti-24. The impact of using material properties obtained under static loading conditions is anticipated to be small. The rationale for this assumption is that the mechanical properties of subject materials do not significantly change at the peak strain rates that occur during the rockfall. This assumption is used in Section 6.2.2.4.

\subsubsection{Deformation of Drip Shield Vertical Section}

The vertical section of the drip shield is assumed to deform like a cantilever beam with the free edge at the bottom and the fixed edge connected to the curved section at the top. The rationale for this assumption is that the drip shield bottom end has similar boundary conditions to the free end of a cantilever beam, while the connection between the curved section and the vertical section of the drip shield has enough stiffness to prevent excessive deformation. Although the curved section will deform towards the center of its curvature, the effect of this deformation on the results is small compared to the deflection on the vertical section caused by the lateral active pressure. This assumption is used in Section 6.2.2.4.

The drip shield sidewalls are assumed to be unconstrained in the lateral direction during the postclosure period. The rationale for this assumption is that the gantry rail is made of steel sets (BSC 2004 [DIRS 166363], Attachment A-1), which are not anticipated to remain intact during the postclosure period. This assumption is used in Section 6.2.2.4.

Assumption 5.2.16.8 is not valid, nor needed, if natural backfill is used or if substantial natural backfilling occurs. 


\subsection{THERMAL ANALYSIS ASSUMPTIONS}

Thermal analyses are performed at different geometric scales including repository scale, drift scale, waste package scale, and fuel assembly scale (Section 6.3.2). Assumptions appropriate to each scale of thermal analysis are presented in the following sections.

\subsubsection{Assumptions for Repository-Scale Thermal Analyses}

Assumptions used in repository-scale thermal analyses are discussed below.

\subsubsection{Pillar Representation of Repository}

In the pillar representation of the repository, the problem domain is represented as a rectangular parallelepiped (Section 6.3.2). Vertically, it ranges downward from the top of the mountain to well into the saturated zone. Laterally, the representation is bounded by planes parallel to the drifts and centered at the midpoint between the drifts. The thermal boundary conditions at these locations are adiabatic surfaces. For the three-dimensional pillar representation, the axial boundaries are placed perpendicular to the drift axis, either between waste packages or axially bisecting one or both of the waste packages at the end of the drift segment. Again, the thermal boundary conditions at these planes are adiabatic surfaces. The rationale for this assumption is that it approximates a drift segment at or near the geometric center of the repository. The assumption of no lateral heat transfer is appropriate because it maximizes the temperatures within the pillar; however, it is a good assumption only from emplacement to about 1,000 years after, at which time appreciable cooling begins from the edges of the repository (CRWMS M\&O 1994 [DIRS 142611], p. 7). This assumption is used in Sections 6.3.2.4 and 6.3.2.5.

\subsubsection{Omission of Gross Water Movement}

The effect of water mobilization into the repository from the surrounding rock matrix, as well as that from percolation flux that reaches the repository horizon from the surface, is neglected in thermal evaluations of the waste package. The rationale for this assumption is that it is conservative (higher peak temperatures) because it neglects thermal energy transport away from the drifts by the gross movement of this water. For the first few decades after repository closure, the thermal pulse penetrates only a few meters into the host rock and little water will be mobilized. For high temperature operating mode, peak temperatures occur in this same short time period. For low temperature operating mode, the host rock remains below the boiling point of water and no water is mobilized. Hence, neglecting the movement of water has little effect on thermal calculations for either high or low temperature operation.

While gross water movement within the host rock fracture network is not represented, the thermal transport properties include the effect of entrapped water. For instance, rock strata specific heats (DTN: SN0307T0510902.003 [DIRS 164196]) are represented as constant values for temperatures below boiling and are higher than any of the constituents; hence, they include the effect of local water. Near the boiling temperature in the host rock, the specific heat is adjusted upward to account for the latent heat of vaporization of water in the host rock (Figure 5(a)). The thermal conductivity is also reduced at rock temperatures above boiling to represent the loss of aqueous water (Figure 5(b)). 
This assumption is used in Sections 6.3.2.4 and 6.3.2.5.

(a)

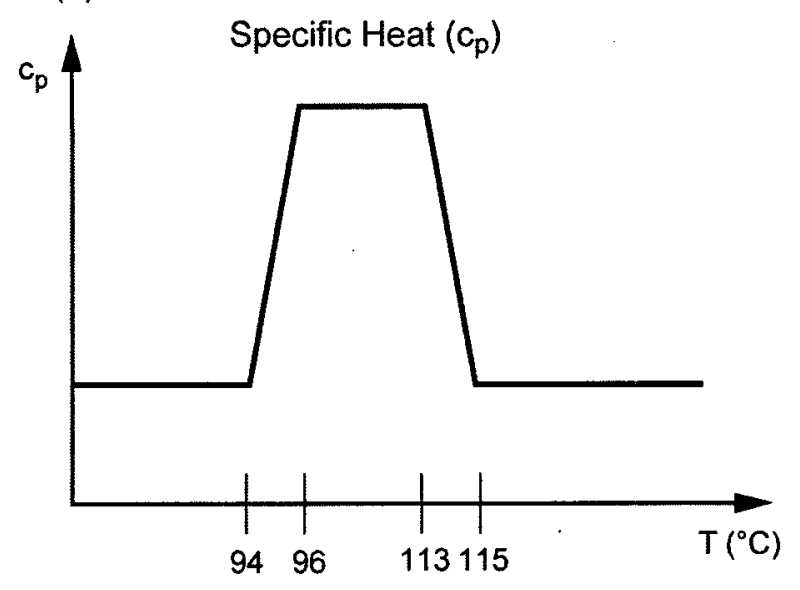

(b)

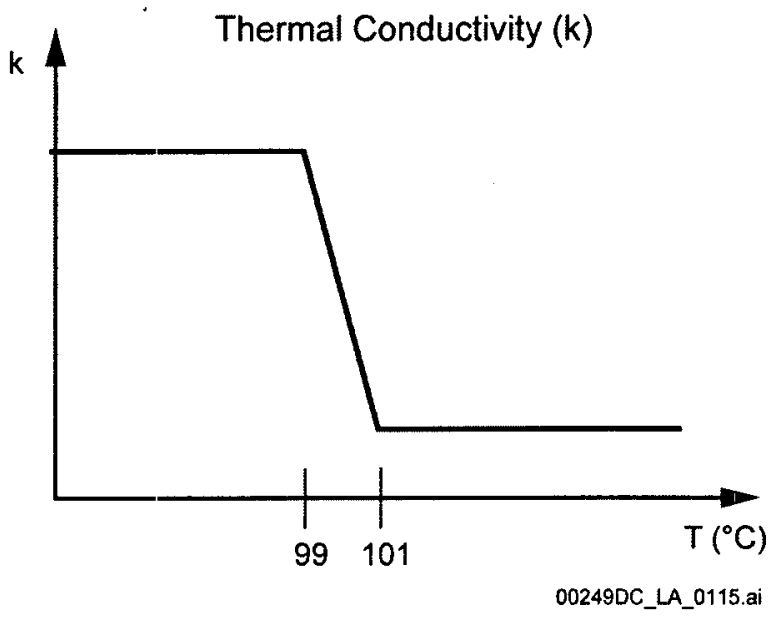

Figure 5. Adjustments of Host Rock Therrnal Transport Properties

\subsubsection{Treatment of Waste Package Internals}

The waste form, basket, and basket support structure within the waste package are represented in repository-scale calculations as a homogeneous, smeared-property, heat-generating cylinder. The length of the cylinder corresponds to the inside length of the inner vessel, and the diameter of the cylinder corresponds to the inner diameter of the inner vessel. The rationale for this assumption is that the internal temperatures are not of immediate interest in repository-scale calculations, provided that the thermal transport properties for the internals are correct in an average sense. This assumption is used in Sections 6.3.2.4 and 6.3.2.5.

\subsubsection{Fixed Temperature at the Surface of the Mountain}

The boundary condition at the top of the two- and three-dimensional repository representations is a fixed temperature, the value of which will be taken from the Technical Data Management System. The rationale for this assumption is that while climatic changes affect the temperature distribution a few meters into the mountain, the rock acts as a thermal capacitor, and the annual averaged surface temperature is adequate for determining temperatures at the repository horizon. This assumption is used in Sections 6.3.2.4 and 6.3.2.5.

\subsubsection{Initial Temperature Gradient in the Mountain}

The initial thermal gradient in the host rock is assumed to be that listed in Table 4. The last row of the table is modified to reflect the assumption that the last gradient reported in the reference extends to the lower bound of the ANSYS representation (a depth of $1300 \mathrm{~m}$, corresponding to the water table depth). The rationale for this assumption is that this gradient is based on a representation gradient profile obtained from the USW G-4 borehole and is typical for Yucca Mountain (Sass et. al. 1988 [DIRS 100644]; DTN: GS950308319213.002 [DIRS 162990]). Also, the temperature gradient below $541 \mathrm{~m}$ depth is not known, and is therefore conservatively 
assumed as stated above. Choosing the lower boundary with a depth of $1300 \mathrm{~m}$, which is far enough from the heat source and the areas of concern, will not significantly affect the repository temperature calculation. This assumption is used in Sections 6.3.2.4 and 6.3.2.5.

Table 4. Initial Temperature Gradient in Host Rock

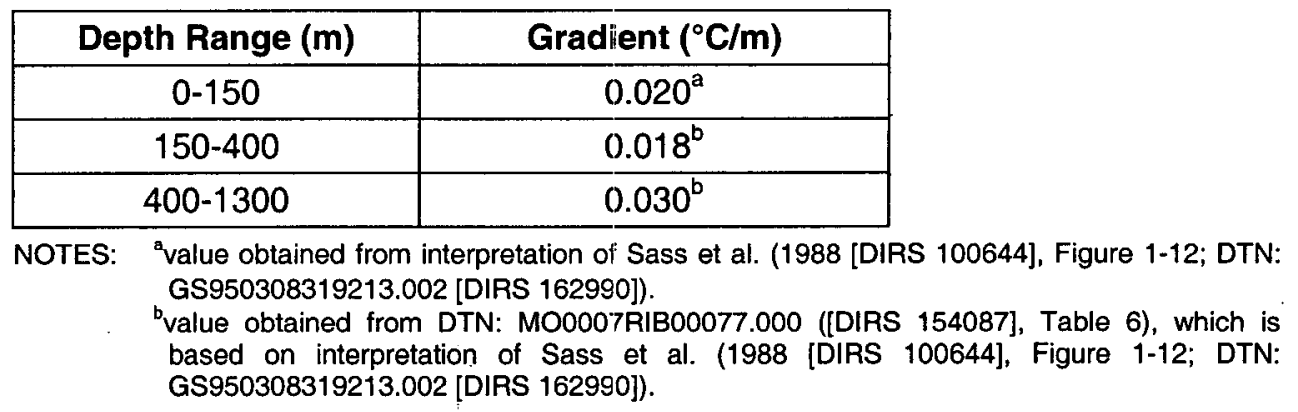

\subsubsection{Modes of Heat Transfer within the Drift}

All three modes of heat transfer (conduction, convection and radiation) occur in the drift. During the preclosure period, most of the heat is removed by mixed (forced and natural) convection. This heat is removed by the ventilation system and the heat transfer to the rock is reduced. The method of calculating convection effects during preclosure is described in Section 5.3.1.7.

During postclosure, convective heat transfer is neglected, and heat transfer is represented by radiation and conduction only. The rationale for this assumption is that neglecting convective heat transfer will result in a conservative calculation of waste form peak temperatures. Calculations in a drift above borehole-emplaced waste packages have shown that radiative heat transfer is an order of magnitude greater than the convective heat transfer (Gartling et al. 1981 [DIRS 142640], p. 59). The dominance of radiation heat transfer can also be shown by simple analytic solutions. Neglecting convection gives conservative (high) values for waste package and cladding temperatures and only slightly lower rock temperatures.

The conductive heat transfer between the waste package and the emplacement pallet, and hence through the pallet into the invert, is neglected. The rationale for this assumption is that the emplacement pallet contacts the waste package in only a few places (an indeterminate number due to the evolving design), and conduction is necessarily limited. An effective thermal conductivity with directional dependence is used to represent the invert.

The modes of heat transfer in each portion of the repository-scale, two-dimensional representation of the drift and near-field rock are presented in Figure 6 for designs that include or omit backfill. The extension to the three-dimensional analyses is obvious.

This assumption is used in Sections 6.3.2.4 and 6.3.2.5. 


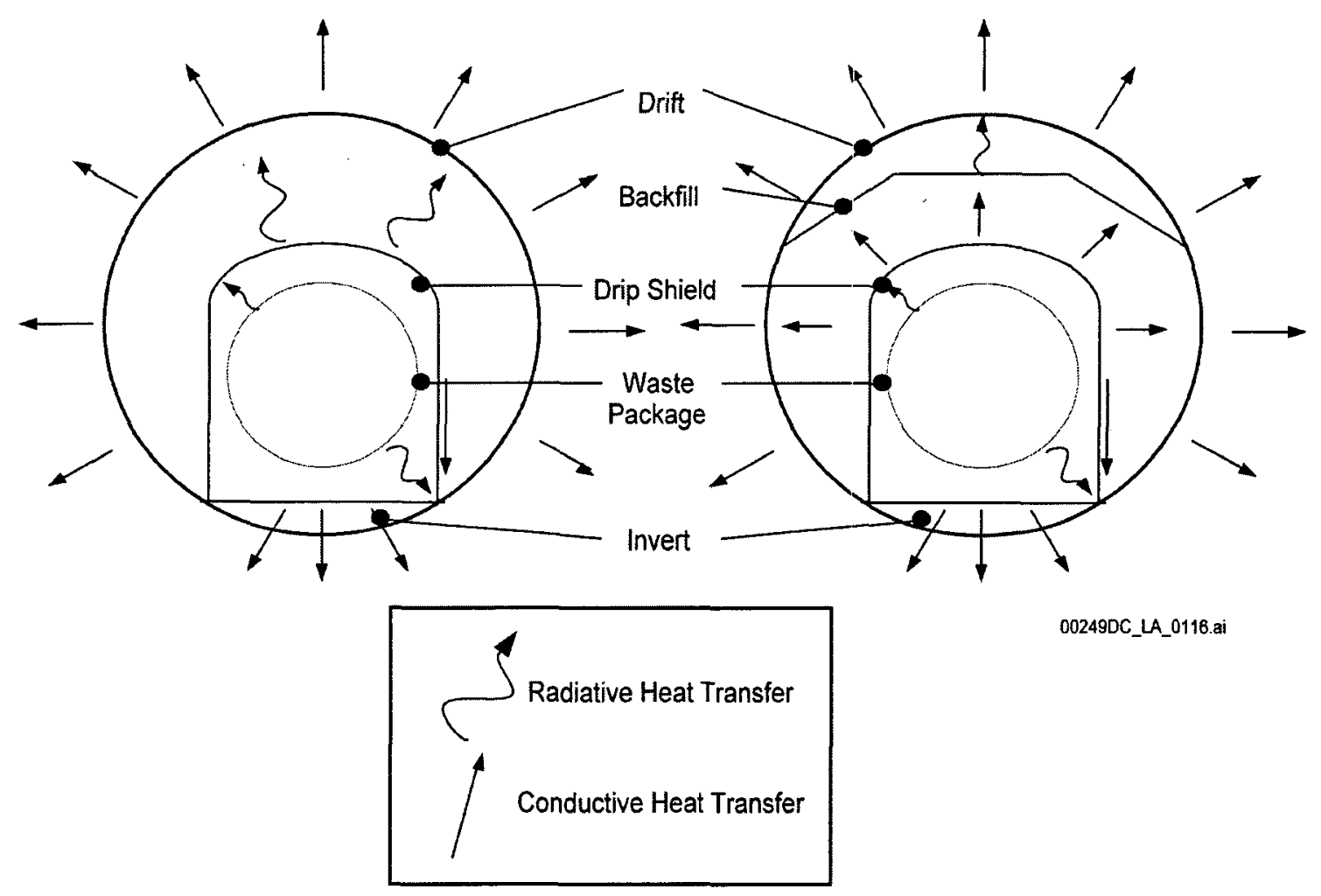

Figure 6. Modes of Heat Transfer In and Near Drift

\subsubsection{Approximation of Heat-Removal by Ventilation}

For repository-scale thermal analysis, convective heat transfer is not explicitly represented in the drift. All heat transfer from the waste package to the drift wall is assumed to be by thermal radiation. During preclosure, a reduction in heat generation is used as a proxy for forced convective heat transfer. The rationale for this assumption is that, while it is slightly non-conservative because it reduces the surface temperature of the waste package, it transfers the correct amount of thermal energy to the drift wall. During postclosure all heat generated in the waste package is transferred by thermal radiation. This approximation increases waste package surface temperature and transfers the correct amount of heat to the drift wall. These assumptions are used in Sections 6.3.2.4 and 6.3.2.5.

\subsubsection{Assumptions for Drift-Scale Thermal Analysis}

All of the assumptions for repository-scale analysis are also applicable to drift-scale analysis. In addition, drift-scale analysis assumes uniform temperature around a circumference $16 \mathrm{ft}(5 \mathrm{~m})$ into the drift rock surface. Note drift-scale analysis is valid only for the same linear heat load as used to determine the 16- $\mathrm{ft}(5-\mathrm{m})$ rock temperature in the corresponding pillar analysis. The rationale for this assumption is drift internal structures have little impact on rock temperatures at this location (CRWMS M\&O 1997 [DIRS 101538], Appendix A). This assumption is used in Section 6.3.2.3. 


\subsubsection{Assumptions for Waste Package-Scale Thermall Analysis}

Assumptions used in waste package-scale thermal analysis are discussed as follows.

\subsubsection{Two-dimensional Representation of Waste Package Internals}

Two-dimensional representations of the waste form and waste package components are used for the purpose of defining the peak-fuel cladding temperatures. Inherent to this assumption is that axial heat transfer does not significantly affect the solution. The rationale for this assumption is that the metal thermal conductivities and heat generation rate distributions are such that axial heat transfer is negligible. This characteristic behavior is shown in The TN-24P PWR Spent-Fuel Storage Cask: Testing and Analyses (Creer et al. 1987 [DIRS 136937]) and Emplacement Scale Thermal Evaluations of Large and Small WP Designs (CRWMS M\&O 1995 [DIRS 106058]). This assumption is used in Section 6.3.2.4.

\subsubsection{Omission of Convection Within the Waste Package}

Convective heat transfer through the waste package fill gas (within the basket gaps and all other waste package vacancies) is neglected. Considering only conduction and radiation heat transfer is assumed to provide conservative results for peak fuel cladding temperature. The rationale for this assumption is as follows: some convective heat transfer will occur in the waste package fill gas; however, in a horizontal emplacement configuration, convection is minor compared to thermal radiation (at the expected temperatures), and stable convection cells either do not develop or are difficult to predict. Also, some fill gases, such as helium, have poor buoyancy relative to their thermal conductivity (unlike air, for example), and natural convection has a negligible contribution to total heat transfer. An extensive discussion of natural convection heat transfer is contained in Introduction to Heat Transfer, $3^{\text {rd }}$ Edition (Incropera and DeWitt 1996 [DIRS 107784], pp. 448-478). This assumption is used in Sections 6.3.2.4 and 6.3.2.5.

\subsubsection{Assumptions for Fuel Assembly-Scale Thermal Analysis}

Assumptions used in fuel assembly-scale thermal analysis are discussed as follows.

\subsubsection{Boundary Conditions for Fuel Assembly Calculations}

Fuel assembly thermal calculations use a quarter-symmetric representation of the assembly. Uniform temperatures are applied at the outer boundaries and adiabatic surfaces are used at the inner boundaries. The rationale for this assumption is that the hottest commercial SNF assemblies are in the center of a waste package where the temperature gradients are lowest. This assumption is used in Sections 6.3.2.4 and 6.3.2.5.

\subsubsection{Generation of All Decay Heat in $\mathrm{UO}_{2}$}

Heat generation due to the decay of commercial SNF fission products and actinides is assumed to be evenly distributed throughout all of the $\mathrm{UO}_{2}$ pellets. Radial power profiles within the pellets and within the assembly are neglected, and heat generation in the cladding (and other non-fuel hardware) due to activation and radiation energy is ignored. Heat generated by fission products in the gas gap is distributed through the pellet. The rationale for this assumption is that 
it is conservative to assume that all of the heat produced by the commercial SNF assembly is produced in the $\mathrm{UO}_{2}$ pellet and not the cladding. The temperature distribution within the pellet and cladding is discussed in Determination of PWR Spent Nuclear Fuel Assembly Effective Conductivity (BSC 2003 [DIRS 162416], Attachment V), and it is demonstrated that the highest cladding temperatures result when all the heat is generated in the pellet. This assumption is used in Sections 6.3.2.4 and 6.3.2.5.

\subsubsection{Geometry of Commercial Spent Nuclear Fuel Assembly}

Components of the commercial SNF assembly (cladding, guide tubes, etc.) are assumed not to touch the basket wall or each other. Some spent fuel rods may have bowed such that they contact each other or the basket (or the channel for BWR fuel). The rationale for this assumption is that it is conservative because the only heat transfer is through the fill gas by thermal radiation and conduction. This assumption is used in Section 6.3.2.1.

\subsubsection{Omission of Fuel Irradiation Effects}

Fuel irradiation effects are neglected for the purpose of determining material properties in the commercial SNF assembly representations. The irradiation of $\mathrm{UO}_{2}$ pellets induces several changes in the porosity, composition, and stoichiometry of the fuel. These changes, however, are generally small in light water reactors. Introduction of fission products, burnup, and material cracking under thermal cycling lead to a slight decrease in the thermal conductivity of the fuel; however, these effects are neglected. Also neglected are the effects of fuel densification, swelling, restructuring, and plutonium content; and the oxygen-to-metal ratio of the uranium fuel is assumed to be the theoretical value of two. Zircaloy thermal conductivity is primarily a function of temperature; however, other characteristics, such as residual stress levels, crystal orientation, and minor composition differences (i.e., zircaloy- 2 versus zircaloy-4 as cladding material) may have secondary influences on conductivity. These effects, as well as cladding dimensional changes (e.g., creepdown, thermal expansion, elastic deformation, and stress irradiation growth) are neglected. The rationale for these assumptions is that while these neglected effects may affect temperatures within the pellet, they have little or no impact on the cladding temperature, the parameter for which margin must be demonstrated. This assumption is used in Section 6.3.2.1.

\subsubsection{Omission of Cladding Crud}

Appropriate emissivity values for light water fuel cladding without layers of crud are assumed. The rationale for using these values is that crud generally increases emissivity values, and crud-free oxide thicknesses provide appropriately conservative cladding temperatures. This assumption is used in Section 6.3.2.1.

\subsubsection{Oxide Thicknesses}

The nominal zirconium-oxide thickness for PWR fuel is $50.8 \mu \mathrm{m}$ ( 2 mils) and that for BWR fuel is $101.6 \mu \mathrm{m}$ ( 4 mils). These values are typical of fuel that has been exposed in commercial light water reactors. From Waste Form Characteristics Report, Revision 1 (Stout and Leider 1997 [DIRS 100419], p. 2.1.3.1-3), $50 \mu \mathrm{m}(\sim 2 \mathrm{mils})$ is a conservative maximum for PWR commercial 
SNF with a discharge exposure of about $40 \mathrm{GWd} / \mathrm{MTHM}$ (gigawatt-days/metric tons heavy metal). BWR environments induce a significantly thicker cladding oxide layer in the 40 to 50 GWd/MtHM exposure range. The $101.6 \mu \mathrm{m}$ thickness represents an upper bound based on BWR design experience as described in Determination of $7 x 7$ Rod Array BWR SNF Assembly Effective Thermal Conductivity (CRWMS M\&O 1996 [DIRS 144269]), Determination of 8x8 Rod Array BWR SNF Assembly Effective Thermal Conductivity (CRWMS M\&O 1996 [DIRS 144270]), and Determination of $9 x 9$ Rod Array BWR SNF Assembly Effective Thermal Conductivity (CRWMS M\&O 1996 [DIRS 144271]). This assumption is used in determining decay for Section 6.1.2.

\subsubsection{Assumptions for Waste Package Fire Analyses}

Calculations for fire analyses are performed parametrically, assuming the worst fire conditions will not exceed those for transportation casks. Fire controls are anticipated to result in far less severe fire conditions. An axisymmetric, finite element representation of the waste package is used. The waste package inner components are integrally connected and fuel assemblies are modeled with an effective thermal conductivity. The integral connection provides minimal thermal resistance to the fire and is therefore conservative in estimating peak clad temperatures from a fire. More detailed assumptions are given in reports for fire analysis of each type of waste package. This assumption is used in Section 6.3.2.

\subsubsection{Assumptions for Waste Package/Surface Facility Analyses}

Thermal analyses of the loaded waste packages in the surface facilities are performed. This assures cladding temperature limits are not violated and that waste package surface temperatures in the weld zone do not exceed temperatures acceptable for welding Alloy 22 N06022. The analyses use an axisymmetric, finite element representation for the waste package and transporter. The concrete welding cell is represented in three dimensions with concrete walls at ambient temperature. The initial temperature of the spent fuel is taken from representative transportation cask reports. Only radiation heat transfer is considered. Convection cooling is conservatively neglected. The rationale for this assumption is that it results in conservative values for peak cladding temperature. This assumption is used in Section 6.3.2.

\subsubsection{Assumptions for DRIFTFLOW Calculations}

DRIFTFLOW is used to determine the effect of ventilation in the repository drifts. All modes of heat transfer (conduction, radiation, and convection) are considered. An overall convection heat transfer coefficient is used. Conduction is calculated by superimposing temperature responses to yearly pulses of heat. The temperature responses are based on ANSYS results for a "pillar" analysis. The rationale is that mathematical solutions can be superimposed and overall heat transfer coefficients are common practice in heat transfer calculations. This assumption is used in Section 6.3.2.

\subsubsection{Assumptions for FLUENT Calculations}

Convection heat transfer coefficients are calculated by Computational Fluid Dynamics analysis. Ventilation flow rates are specified and a uniform rock temperature $16 \mathrm{ft}(5 \mathrm{~m})$ from the surface 
are taken from ANSYS calculations. The rationale for this is that previous calculations have shown that drift internal structures have little impact on rock temperatures at this location (CRWMS M\&O 1997 [DIRS 101538], Appendix A). This assumption is used in Section 6.3.2.3.

\subsection{SHIELDING ANALYSIS ASSUMPTIONS}

Assumptions used in performing shielding analysis are discussed in this section.

\subsubsection{Use of an Axial Peaking Factor}

Because the radiation source terms are generated with the assumption that the burnup is uniformly distributed within a SNF assembly, an axial power peaking factor is used to develop neutron and photon source strengths in the active fuel region. The rationale for this assumption is to conservatively account for the maximum values of the actual axial source distributions. The axial power peaking factor of a PWR SNF assembly is 1.25 (BSC 2004 [DIRS 169593], Section 5.2.1]. This value is based on the predicted axial decay heat rate profile of a PWR SNF assembly provided in Testing and Analyses of the TN-24P PWR Spent-Fuel Dry Storage Cask Loaded with Consolidated Fuel (EPRI 1989 [DIRS 101947], p. 3-26). The axial power peaking factor of a BWR SNF assembly is 1.25 (BSC 2003 [DIRS 166596], Section 3.5, page 9). This peaking factor has been determined from the axial burnup profile of a BWR SNF assembly as a function of average assembly burnup (BSC 2003 [DIRS 164364], p. 47). The rationale for using this value is that it is conservative for an assembly average burnup of 40 GWD/MTU or higher. This assumption is used in the shielding analysis discussed in Section 6.4.2.

\subsubsection{Homogenization of the Radiation Source Region for Commercial Spent Nuclear Fuel}

In a three-dimensional shielding analysis for the waste packages containing commercial SNF, the contents and radiation sources of each SNF assembly region (i.e., plenum, end fitting, and active fuel) are uniformly homogenized. The rationale for this assumption is based upon a study of the effect of source geometry on the waste package surface dose rates described in Calculation of the Effect of Source Geometry on the 21-PWR WP Dose Rates (CRWMS M\&O 1998 [DIRS 102134 ], pp. 22 to 26). The results of the study indicate that identical dose rates on the external surfaces of a waste package are obtained for two different source geometry representations: a detailed geometric representation, and a representation in which the contents and radiation sources are homogenized inside region dimensions. This assumption is used in the shielding analysis discussed in Section 6.4.2.

\subsubsection{Homogenization of the U.S. Department of Energy Spent Nuclear Fuel Canister}

The contents and radiation source of the DOE SNF canisters are homogenized inside the cavity of the DOE SNF canister. However, if the DOE SNF canister contains one intact SNF assembly (e.g., Shippingport Light Water Breeder Reactor SNF), the assembly contents and radiation sources are homogenized inside the assembly dimensions. The rationale for this assumption is that the homogenization process decreases the fuel self-shielding and moves the radiation source closer to the outer surfaces of the waste package, allowing more particles to reach the outer 
surface and, hence, increasing the dose rate. This assumption is used in the shielding analysis discussed in Section 6.4.2.

\subsubsection{Omission of Waste Package Internals}

For the one-dimensional shielding analysis of waste package radial dose rates, the fuel region of the waste package, which consists of the waste form, neutron absorber plates, thermal shunts, and other structural members, is radially homogenized inside the waste package cavity with some internal components omitted. The rationale for this assumption is that it is conservative for calculating dose rates on the surfaces of the waste package because the structure components that would otherwise attenuate neutrons and photons are not represented. This assumption is used in the shielding analysis discussed in Section 6.4.2.

\subsubsection{Use of a Watt Fission Spectrum}

A Watt fission spectrum (Briesmeister 1997 [DIRS 103897], Appendix H, pp. H-2 and H-3) is used for the neutron source energy distribution of DOE SNF because the actual neutron spectra are not available for most of the DOE fuels. The rationale for this assumption is that the dose rate evaluation is not sensitive to the neutron spectrum because the neutron dose rate contribution to the total dose rate outside of the waste package is negligible for the repository preclosure period. This assumption is used in the shielding calculations discussed in Section 6.4.2.

\subsubsection{Fresh Fuel Assumption}

The composition of fresh fuel is used to represent the attenuation properties of spent fuel in the shielding calculations. The rationale for this assumption is that, while photon attenuation properties of spent fuel and fresh fuel are similar, fresh fuel has a conservatively higher neutron dose rate, due to greater production of fission neutrons. This is not due to the fission yield for neutrons, but rather to the greater abundance of fissile constituents. The neutron and gamma ray sources in the actinides and fission products are derived from the spent fuel composition and are represented as fixed sources in the shielding calculations. Therefore, the radiation sources are not affected by this assumption. This assumption is used in the shielding calculations discussed in Section 6.4.2.

\subsubsection{Treatment of Trace Elements}

For material compositions having elements with specified ranges (i.e., weight percentages of each constituent), the midpoint value is used and the abundance of the most abundant element is adjusted upward to maintain the material density. The rationale for this assumption is that small weight percentage variations of each element constituent do not affect the accuracy of dose results, as long as the density is maintained. This assumption is used in the shielding calculations discussed in Section 6.4.2.

\subsubsection{High-Level Radioactive Waste Glass Source Terms}

The source terms for the design basis glass developed at the Savannah River Site Defense Waste Processing Facility are assumed for all other HLW glass forms. The rationale for this assumption is that these source terms provide conservative (higher) dose rates for the codisposal waste 
packages because the source terms for the rest of the HLW glass forms are less intense (CRWMS M\&O 2000 [DIRS 151947], Attachments V and VI). This assumption is used in the shielding calculations discussed in Sections 6.1.2.3 and 6.4.2.2.

\subsubsection{Infinite Cylinder Representation of a Waste Package in SAS1 Analyses}

For waste package shielding analysis, SAS1 is an effective tool for evaluating the radiation levels on and beyond the radial outer surface of a waste package. SAS1 assumes a waste package to be an infinite cylinder with a homogenized fuel region in the center, enclosed by the inner vessel and corrosion barrier. The rationale for this assumption is that, because the length of a waste package is approximately three times the diameter, the infinite cylinder representation of the waste package should yield accurate dose results for the radial direction. This assumption is used in Section 6.4.1.4.

\subsection{CRITICALITY ANALYSIS ASSUMPTIONS}

Specific assumptions used in criticality analysis are documented in loading curve evaluations. General assumptions used in performing criticality analyses are given in the following model reports: Errata for Isotopic Model Report for Commercial SNF Burnup Credit (BSC 2003 [DIRS 168037]), Configuration Generator Model for In-Package Criticality (BSC 2003 [DIRS 165629]), Geochemistry Model Validation Report: External Accumulation Model (BSC 2001 [DIRS 156324]), Geochemistry Model Validation Report: Material Degradation and Release Model (BSC 2001 [DIRS 156790]), and Criticality Model Report (BSC 2003 [DIRS 165733]).

\section{WASTE PACKAGE COMPONENT ANALYSIS METHODS AND COMPUTATIONAL TOOLS}

This section describes the analytical methodology and computational tools used in each of the design disciplines. For each discipline, reference is made to specific computational tools and their current qualified versions, to demonstrate that qualified computer codes embodying these methodologies exist. However, this should not be construed to limit subsequent analyses and calculations to only these versions. New versions of these computer codes can and will be qualified for future analyses and calculations.

Although this section does not discuss them, there are standard engineering approaches applicable to various problems. This report does not provide estimates of any of the factors for the Postclosure Safety Case or Potentially Disruptive Events.

\subsection{SOURCE TERM DETERMINATION}

The following describes the generation of source terms for the commercial SNF, DOE SNF, and HLW. The method for calculating DOE SNF and HLW source terms differs from that for the commercial SNF, as the information available for these waste streams is considerably different from that for commercial SNF. For a commercial SNF assembly with any given enrichment, burnup, and cooling time, a burnup calculation can be performed that reasonably simulates the irradiation history of the assembly in the reactor core and the subsequent decay after it is removed from the reactor. For DOE SNF, the source term methodology is described in Source Term Estimates for DOE Spent Nuclear Fuels (DOE 2003 [DIRS 163377]). For the HLW, the 
chemical composition (in the case of the HLW) and the estimated radionuclide inventory at a certain year are provided. The source terms for these waste forms can be computed by simply decaying the radionuclides to the desired times. The methodology and computational tools used to generate the source terms are presented in more detail in the following sections.

\subsubsection{Computational Tools}

Oak Ridge National Laboratory developed the SCALE code system for the NRC to satisfy the need for standardized analysis methods for licensing evaluations of nuclear fuel facilities and package designs. The SCALE system is a collection of well-established functional modules (computer codes) that can be used individually or in combination to perform criticality, shielding, and heat transfer analyses. The system has many control modules, each of which combines several functional modules into analysis sequences to perform a specific analysis. The SAS2H control module and the ORIGEN-S functional module in the SCALE system are the primary computational tools for source term generation.

\subsubsection{Description of SAS2H for Commercial Spent Nuclear Fuel Source Term Generation}

In depletion analyses, the fuel isotopics change with time and are significantly different between fuel cycles. Hence, the fuel isotopics and their macroscopic cross sections must be updated to reflect these changes. For each time-dependent fuel composition, SAS2H performs onedimensional neutron transport analyses of the fuel assembly using a two-part procedure with two separate lattice-cell representations. The first representation (Path A) is a unit fuel-pin cell from which cell-weighted cross sections are obtained. The cell-weighted cross sections from this calculation are used in a second representation of a larger unit-cell (Path B) that represents the entire assembly within an infinite lattice. The zones in Path B can be structured for different types of BWR or PWR assemblies containing water rods, burnable poison rods, gadolinium fuel rods, etc. The fuel neutron flux spectrum obtained from Path B is used to update the nuclide cross sections for the specified burnup-dependent fuel composition. The updated cross sections are then used in a point-depletion computation to produce the burnup-dependent fuel composition to be used in the next spectrum calculation. This sequence is repeated over the entire irradiated history of the assembly. An example of these representations for a BWR assembly is presented in Figure 7.

The functional modules executed by SAS2H to carry out the depletion analysis are BONAMI-S, NITAWL-II, XSDRNPM-S, COUPLE, and ORIGEN-S. BONAMI-S and NITAWL-II perform resonance self-shielding analyses of the cross sections in each irradiation cycle. XSDRNPM-S performs the one-dimensional neutron transport analyses in Path A and Path B. COUPLE updates the cross-section constants of all nuclides in the ORIGEN-S nuclear information library with the cell-weighted information and the weighting spectrum from XSDRNPM-S. ORIGEN-S calculates the fuel depletion in all cycles and the decay of nuclides at the completion of fuel irradiation. A more detailed description of ORIGEN-S is provided in the next section. The computational flow diagram in SAS2H for commercial SNF source term generation is presented in Figure 8. 


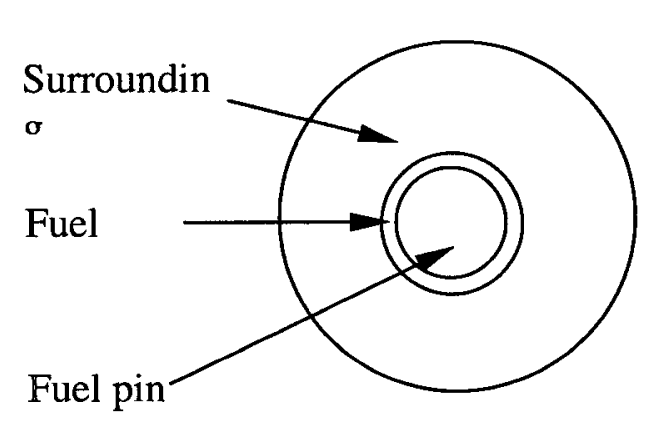

Path A

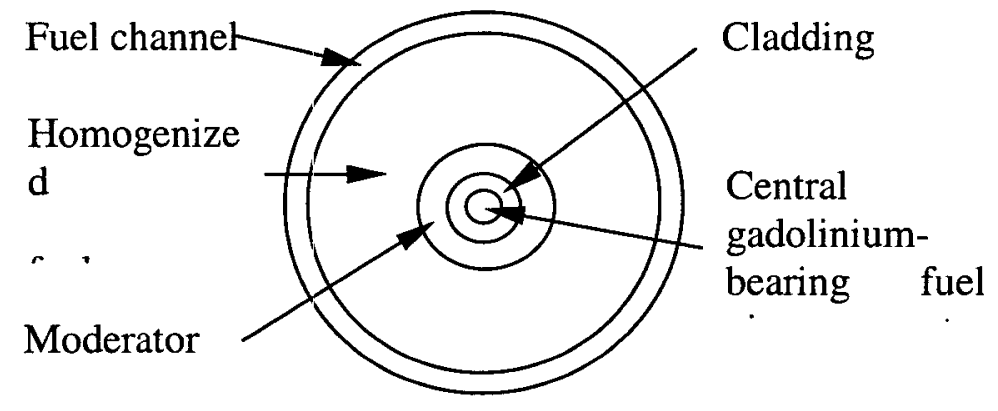

Path B

Figure 7. SCALE Representation of the Fuel Pin Cell and Assembly for SAS2H Calculations

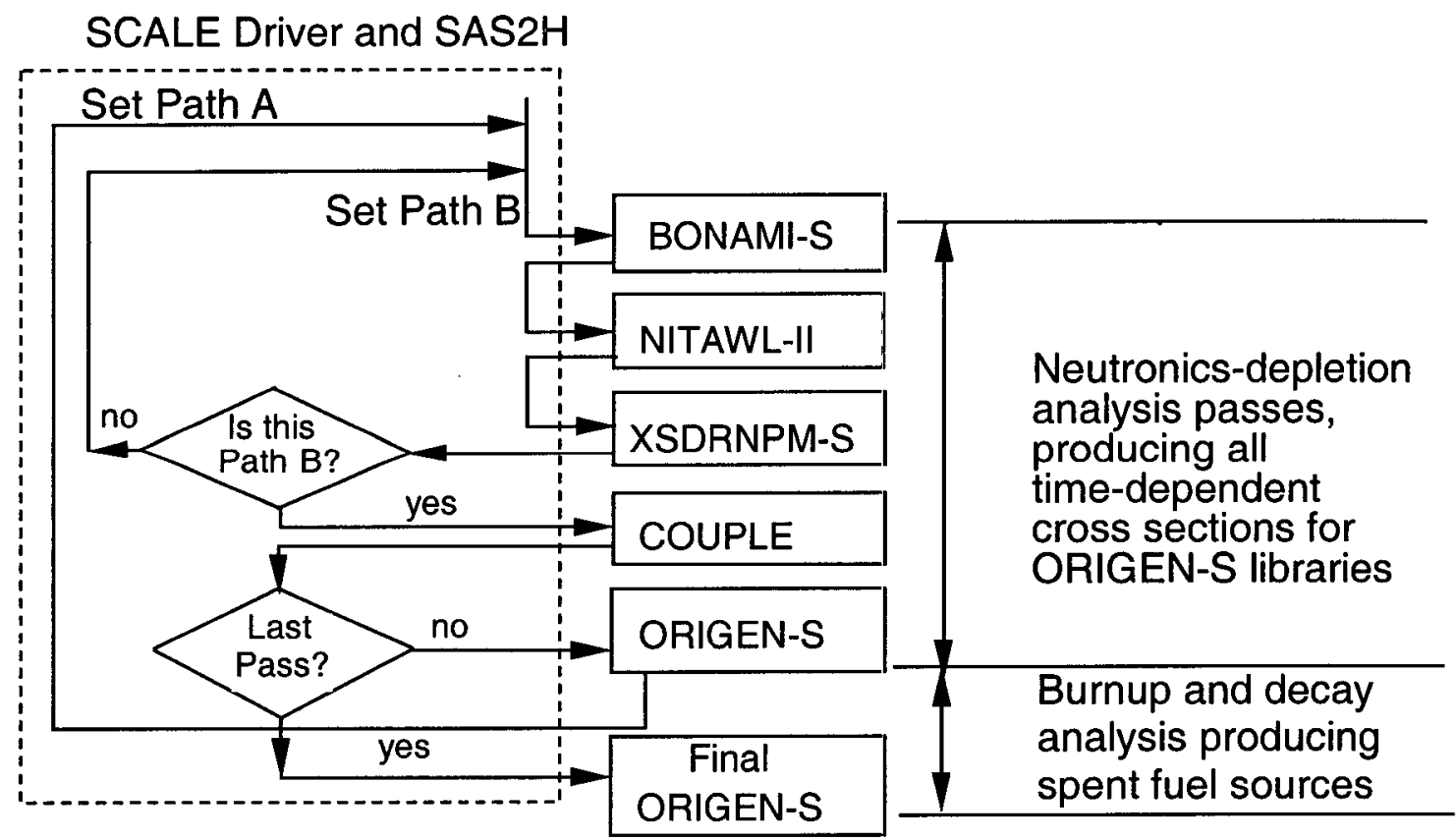

Figure 8. Calculational Flow for Source Term Generation

The source terms for DOE SNF and HLW are not calculated with the SAS2H control module because the radionuclide inventories are given for these fuels. Source terms for DOE SNF and HLW come from Source Term Estimates for DOE Spent Nuclear Fuels (DOE 2003 [163377]), TRIGA (UZrH) Fuel Characteristics for Disposal Criticality Analysis (DOE 1999 [DIRS 103891] and Source Terms for HLW Glass Canisters (CRWMS M\&O 2000 [DIRS 151947]). The inventories are entered directly into ORIGEN-S to be decayed to the desired times. This is similar to the final ORIGEN-S case for the commercial SNF. In the case of HLW, the radionuclide inventory is mixed with the glass chemical composition and then decayed. 


\subsubsection{Description of ORIGEN-S}

ORIGEN-S is the main functional module of SAS2H (the control module of the SCALE code system) that carries out the depletion and decay calculations. It can also be used as a stand-alone program. ORIGEN-S computes time-dependent concentrations and source terms of a large number of isotopes, which are simultaneously generated or depleted through neutronic transmutation, fission, radioactive decay, input feed rates, and physical or chemical removal rates.

ORIGEN-S can use three kinds of cross-section libraries: card image libraries with nuclear and photon yield information, binary libraries with nuclear transition and photon yield information, and the Master Photon Data Base containing detailed photon energy and intensity data. The second of these, the binary library, contains the same type of information that the card-image libraries do, but for only one kind of problem. This represents a major advantage over the card image libraries in that these cross sections can be replaced with those determined from the detailed neutronics calculations performed by the functional modules that precede ORIGEN-S in the SAS2H module. This means that rather than using a previously defined cross-section set, the code can be used in conjunction with the cross-section processing codes of SCALE that create problem-specific libraries. This capability has led to the NRC preference for ORIGEN-S. The following is an excerpt from NUREG-1536 (NRC 1997 [DIRS 101903], p.5-3):

"Generally, the applicant will determine the source terms using ORIGEN-S (e.g., as a SAS2 sequence of SCALE), ORIGEN2, or the DOE Characteristics Data Base. Although the latter two are easy to use, both have energy group structure limitations.... If the applicant has used ORIGEN2, verify that the chosen crosssection library is appropriate for the fuel being considered. Many libraries are not appropriate for a burnup that exceeds $33,000 \mathrm{MWd} / \mathrm{MtU} . "$

This statement derives from the fact that previous cornpilations of source term values used ORIGEN2 and relied on previously calculated cross-section libraries of limited information, which can easily be used outside the applicable range. These libraries are only appropriate for fuels that have undergone certain irradiation histories and are not as accurate as the problemspecific libraries generated for ORIGEN-S.

\subsubsection{Description of Pertinent Analyses}

\subsubsection{Commercial Waste Forms}

The thermal output, radionuclide inventories, and radiation spectra for commercial SNF are developed according to the source term methodology described in this document. Radiation source terms for commercial SNF have been created for PWR and BWR fuels. These are documented in:

- PWR Source Term Generation and Evaluation (BSC 2004 [DIRS 169061])

- BWR Source Term Generation and Evaluation (BSC 2003 [DIRS 164364]). 
The commercial SNF source terms calculated with the representative PWR and BWR assemblies can be interpolated to provide reasonably close approximations for all assemblies in the waste stream. It is assumed that these interpolated source terms are comparable to those that would have been obtained from a detailed calculation for each assembly. The waste stream source terms are developed by convoluting source terms for PWR and BWR assemblies with detailed assembly information for a specified waste stream. They are documented in Waste Packages and Source Terms for the Commercial 1999 Design Basis Waste Streams (CRWMS M\&O 2000 [DIRS 138239]).

\subsubsection{U.S. Department of Energy Spent Nuclear Fuel}

The total initial radionuclide inventory provided by Idaho National Engineering and Environmental Laboratory (DOE 2003 [163377]) for the year 2010 can be used to calculate the total radionuclide inventory and the source terms for the average DOE SNF canisters for the time period out to one million years. The ORIGEN-S program is used to perform the decay calculations. The following two documents provide the results of calculations based on earlier data (DTN: MO0001SPADBE00.001 [DIRS 146362]):

- Radionuclide Inventories for DOE SNF Waste Stream and Uranium/Thorium Carbide Fuels (CRWMS M\&O 2000 [DIRS 153247])

- Source Terms for Average DOE SNF Canisters (CRWMS M\&O 2000 [DIRS 153345])

- Inventory and Characteristics of Potential Repository Waste (BSC 2004 [DIRS 167441])

\subsubsection{Defense High-Level Radioactive Waste}

According to Waste Acceptance System Requirements Document (DOE 2002 [DIRS 158873], Section 5.4.1.B(2)), the producers of HLW are required to report the estimated total and individual canister inventory, and the associated uncertainties, of radionuclides (in curies) that have half-lives longer than 10 years and that are, or will be, present in concentrations greater that 0.05 percent of the total radioactive inventory indexed to the years 2010 and 3110 . This may become an operational requirement not connected to the initial inventories supplied by the various sites. The time-dependent photon and neutron sources, decay heat sources, and radionuclide contents and activities of the HLW forms are generated in ORIGEN-S decay calculations using the initial radionuclide inventories and chemical compositions of the HLW forms provided by the producers. These calculations are documented in Source Terms for HLW Glass Canisters (CRWMS M\&O 2000 [DIRS 151947]), which provides time-dependent source terms for HLW.

\subsection{STRUCTURAL DESIGN}

Structural design uses the computational tools discussed in Section 6.2.1 to perform various analysis discussed in Section 6.2.2. A discussion of the mesh used for structural calculations is given in Section 6.2.3. Design stress and ductility limits are presented in Sections 6.2 .4 and 6.2.5. 


\subsubsection{Computational Tools}

Structural calculations are performed using ANSYS, LS-DYNA, and MATHCAD.

\subsubsection{ANSYS}

ANSYS is a finite-element software package that can be used to solve a variety of problems. Waste packages, drip shields, and pallets can be represented as two-dimensional or threedimensional finite-element geometries, depending on the symmetry of the design or the loading. ANSYS is widely used for structural evaluations of static and dynamic problems. Materials can be represented with elastic or elastic-plastic temperature-dependent properties. Dynamic evaluations can be performed, such as real-time events with gravitational acceleration acting on component masses. Interfaces between components are represented with contact elements that incorporate interface stiffness and friction. Seismic evaluations can be performed as frequency domain analyses using a response spectrum or can be solved as time-domain analyses using time histories (acceleration, velocity, or displacement). Thermal expansion and stress can be calculated by combining thermal and structural representations into a single analysis.

\subsubsection{LS-DYNA}

LS-DYNA is a finite element program for nonlinear dynamic analysis of structures in three dimensions. Livermore Software Technology Corporation is the development source for the LS-DYNA finite element analysis software program. LS-DYNA is capable of simulating complex real world problems and is widely accepted as the premier analysis software package for a vast number of engineering applications. LS-DYNA analysis capabilities include, but are not limited to, nonlinear dynamics, rigid multi-body dynamics, quasi-static simulations, thermal analysis, fluid analysis, fluid-structure interactions, and finite element method-rigid multi-body dynamics coupling. LS-DYNA is well suited for performing dynamic impact analyses of the waste packages, drip shields, and emplacement pallets.

\subsubsection{MATHCAD}

MATHCAD can solve systems of equations, allowing the user to evaluate the impact of parameter variance quickly.

\subsubsection{Description of Pertinent Analyses}

Structural calculations demonstrate that the waste package, drip shield, and emplacement pallet meet the requirements for normal operations and event sequences. These fall into the following broad groups:

- Geometric Design

- Normal Operations

- Internal Pressurization

- Impacts on Waste Package or Drip Shield

- Dynamic Impacts on the Waste Package

- Seismic Evaluations 
- Residual Stress Reduction.

\subsubsection{Geometric Design Calculations}

Geometric design calculations are primarily sizing calculations to verify that each component is designed to accept the waste form with the dimensions given in the system description documents. Additionally, external features and features that interface with the Facilities Design Project are discussed and demonstrated to show compliance with the proper criteria. These calculations are written as sections of the analysis of the appropriate component design, rather than as stand-alone calculations.

\subsubsection{Normal Operations Calculations}

Normal operating loads are those associated with expected normal operations, such as loading, maneuvering, and emplacing the waste packages.

Vertical and Horizontal Lifting by Trunnion Ring_Lifting calculations will be performed as three-dimensional, static finite-element analyses for each design configuration. The geometry of the internal structure of the waste packages will be simplified, and symmetries will be taken into account within the representation of the waste packages. Nevertheless, the features of the problem relevant to the structural calculations (overall dimensions, masses, and mechanical properties of the materials) will be preserved.

In the horizontal lifting calculations, the trunnion ring will be taken into account by applying appropriate boundary conditions to the waste package. As for the vertical lifting calculations, a potential/proposed design of the trunnion ring will be included in the finite element representation.

Lifting by Emplacement Pallet-A lifting calculation is performed for the heaviest waste package design loaded on an emplacement pallet. This calculation is performed as a quartersymmetric, three-dimensional, static finite-element analysis using LS-DYNA. This representation includes the waste package as a cylinder of shell elements. A force is applied inside of the cylinder to account for the overall mass of the simplified waste package. This approach preserves all features of the problem relevant to the structural calculation.

Static Loading of Waste Package on Emplacement Pallet-The stresses in the emplacement pallet, due to the static loading of the waste package on the pallet, are assessed using a quartersymmetric, three-dimensional, static, finite-element analysis in LS-DYNA. The waste package is represented as a simple rigid cylindrical shell with an internal force applied to account for the weight of the non-represented parts and internals mass.

The stresses in the waste package, due to static support from the emplacement pallet are also assessed using quarter-symmetric shell elements, three-dimensional, static, finite-element analysis in LS-DYNA. A simplified inner vessel with lids and a simplified outer corrosion barrier with lids are represented on a simplified rigid pallet. An equivalent force applied on the inside of the inner vessel represents the mass that was removed from the inner vessel and outer corrosion barrier. 
Static Load of Collapsed Drift on Drip Shield-The stress and buckling within the drip shield, due to static load of the rock from the collapsed drift, is evaluated using a quarter-symmetric, three-dimensional, static, finite-element LS-DYNA analysis. The drip shield connector plates, connector plate guides, and lifting plates are not included in this representation. This slightly conservative approach has a negligible effect on calculated results. The overburden pressure, which takes into account the masses of the loose rock, is applied statically on appropriate structural members.

Residual and Differential Thermal Expansion Stresses-Residual and differential thermal expansion stresses are evaluated in the axial and radial directions for all waste packages. This stress is evaluated parametrically using the highest projected surface temperature of the waste package near $239^{\circ} \mathrm{C}$ (BSC 2003 [DIRS 164726], Section 6). The inner vessel and outer corrosion barrier are designed with radial and axial gaps to prevent contact that would result from thermal expansion. These gaps are toleranced such that the nominal dimension is the minimum gap allowed.

\subsubsection{Internal Pressurization Calculations}

The pressurization of the waste package is assumed to occur due to the rupture of all fuel rod cladding or other primary barriers contained in the waste package, provided the waste form retains an intact pressure boundary before being loaded into the waste package. The calculation uses a closed-form solution to the problem of a cylindrical shell subject to internal pressure load to determine the maximum stresses in the waste package. In this evaluation, the inner vessel lid is assumed to fail before the outer lid; however, no structural credit is assumed for the outer lid. Evaluations are performed over uniform waste package temperatures ranging from $20^{\circ} \mathrm{C}$ to $600^{\circ} \mathrm{C}$. The peak stresses (membrane and bending) at the junction of the cylinder and lid from these evaluations are obtained and shown to be less than the ultimate tensile stress.

\subsubsection{Impacts on Waste Package or Drip Shield}

Rockfall on Waste Package-The waste package rockfall is evaluated as a three-dimensional, transient dynamic, elastic-plastic finite-element analysis using LS-DYNA. The interaction of the waste package internals, inner vessel, and outer corrosion barrier is conservatively assumed to maximize the stress on the inner vessel and outer corrosion barrier. A realistic rock geometry is assumed for this evaluation. The rock shape and dimensions are based on rock fracture characteristics, and static material properties are conservatively used due to the unavailability of dynamic material properties. Further conservatism is included by use of material properties at maximum repository temperatures.

The rock may have an initial velocity due to a seismic event and then be accelerated due to gravitational forces until it strikes the waste package surface. The simulation is continued throughout the impact until the rock begins to rebound at which time the induced stresses reach peak values. This approach also provides the results of the rock impact in terms of the residual stresses because the finite element simulation is continued until the steady state values of stresses are obtained. The resulting residual stresses are subsequently used to assess the susceptibility of the drip shield design to stress corrosion cracking. 
Rockfall on Drip Shield-The fall of rocks onto a drip shield is evaluated as a three-dimensional, transient dynamic, elastic-plastic finite-element analysis using LS-DYNA. A realistic rock geometry was assumed. The shape and dimensions of the rocks are obtained from appropriate sources, and static material properties are conservatively used due to the unavailability of dynamic material properties (DTN: MO0301MWD3DE27.003 [DIRS 161536]). Further conservatism is included by use of material properties at maximum repository temperatures.

The relative velocity between the rock and the drip shield includes three different phases prior to impact: initial velocity of the rock toward the drip shield in accordance with the seismic ground motion, the velocity gained by the rock due to gravitational acceleration, and the velocity of the drip shield toward the rock just prior to impact in accordance with the seismic ground motion. The finite element simulation is continued throughout the impact until the rock begins to rebound, at which time the stresses reach peak values and the maximum displacements are obtained. This approach also provides the results of the rock impact in terms of the residual stresses because the finite element simulation is continued until the steady state values of stresses are obtained. The resulting residual stresses are subsequently used to assess the susceptibility of the drip shield design to stress corrosion cracking.

Equipment Impact on End of Waste Package-The fall of handling equipment onto the end of a waste package will be evaluated as a three-dimensional, transient dynamic, elastic-plastic finite-element analysis using LS-DYNA.

Missile Impact on Waste Package-The calculation for missile impact on a waste package is performed as a dynamic, low-velocity impact analysis using basic strength-of-materials relationships and empirical relationships obtained for the impact of projectiles onto plates. The region of missile impact is represented as a flat plate supported at each end. The analysis is for missiles resulting from accident conditions and not for missiles due to terrorism.

\subsubsection{Dynamic Waste Package Impacts}

Vertical Drop-The vertical drop evaluation is performed for a waste package as a three-dimensional, transient dynamic, elastic-plastic finite-element analysis using LS-DYNA. This representation of the waste package is positioned just above an unyielding surface. The interaction of the waste package internals is conservatively assumed to maximize the stress on the inner vessel and outer corrosion barrier. While static and kinetic friction both have negligible effects, approximate friction coefficients are used. Static material properties are conservatively used, due to the unavailability of dynamic material properties.

Swing Down-The swing down evaluation is performed for a waste package as a three-dimensional, transient dynamic, elastic-plastic finite-element analysis using LS-DYNA. The waste package is being lifted in a horizontal orientation at a height of $2.4 \mathrm{~m}$ when the lifting device inadvertently releases one end. One end of the waste package remains held by the lifting device while the other end swings down and impacts the ground.

10-Degree Oblique Drop with Slap Down-The 10 degree oblique drop with slap down evaluation is performed for a waste package as a three-dimensional, transient dynamic, elastic- 
plastic finite-element analysis using LS-DYNA. The waste package is being lifted in a horizontal orientation at a height of $2.4 \mathrm{~m}$ when the lifting device inadvertently releases both ends. The waste package impacts the ground with a 10 degree angle between the ground and the centerline of the waste package then slaps onto the unyielding surface.

Tipover-The tipover evaluation is performed for a waste package as a three-dimensional, transient dynamic, elastic-plastic finite element analysis using LS-DYNA. A representation of the waste package is oriented with an angle from vertical such that the center of mass is rotated past the pivot point, inducing an overturning moment. The waste package representation is then allowed to rotate and impact an unyielding surface.

Tipover from Elevated Surface-This evaluation is performed as a three-dimensional, transient, dynamic, elastic-plastic finite element analysis using LS-DYNA. A representation of the waste package is oriented with an angle from the vertical such that the center of mass is rotated past the pivot point. The pivot point is a surface that is a specified distance above the impact surface. The finite element representation is initiated just before the waste package makes impact, with the appropriate initial conditions. The impact surface is unyielding to ensure conservative results. The problem is solved iteratively until the reported stresses have climaxed.

Horizontal Drop on Flat Surface-The horizontal drop evaluation is performed for a waste package as a three-dimensional, transient dynamic, elastic-plastic finite element analysis using LS-DYNA. A representation of the waste package is positioned just above an unyielding surface. The interaction of the waste package internals and waste package is conservatively assumed to maximize the stress on the inner vessel and outer corrosion barrier. Static and kinetic friction both have negligible effects.

Horizontal Drop with Emplacement Pallet-The horizontal drop with emplacement pallet evaluation is performed as a three-dimensional, transient dynamic, elastic-plastic finite element analysis using LS-DYNA. A quarter-symmetric representation of the waste package and pallet is positioned above an unyielding surface.

Corner Drop-The corner drop evaluation is performed for a waste package as a threedimensional, transient dynamic, elastic-plastic finite-element analysis using LS-DYNA. A full representation of the waste package is oriented with an angle from vertical such that the center of mass is located directly above the corner of impact. In addition, this representation of the waste package is positioned just above an unyielding surface. The interaction of the waste package internals and waste package is conservatively assumed to maximize the stress on the inner vessel and outer corrosion barrier. Static and kinetic friction both have a negligible effect. The striking of the upper end of the waste package after the corner impact is not assessed because that damage is bounded by the tipover analysis from an elevated surface.

Transporter Runaway-Events resulting in a transporter runaway are not credible (BSC 2003 [DIRS 164128], Section 5.6.4), but transporter accidents may occur. For these events, if identified, evaluations will be performed as a three-dimensional, transient dynamic, elasticplastic finite-element analysis using LS-DYNA, in a manner similar to the vertical and horizontal drops. Impact limiters (represented as yielding surfaces) may be required to show compliance with the criteria. 
Sliding and Inertial Effects of Waste Package Contents-Inertial effects of waste package contents are an intrinsic part of dynamic structural calculations performed explicitly by finite element codes. Sliding effects of waste package contents during impacts are evaluated in calculations where specific answers about stresses in the waste package contents are to be determined. Coefficients of friction are used based on the materials and situation. When coefficient of friction has no effect on the answer, it may not be used. When the waste package contents are not specifically under evaluation, those contents are often simplified so that the mass and inertial effects are accounted for but geornetry is simplified. A rationale for simplifying waste package contents is to decrease computer execution time and size of the finite element representation. Another rationale is that for evaluation of the outer corrosion barrier, waste package contents need not be modeled in detail to get an accurate answer.

\subsubsection{Seismic Evaluations}

Evaluation of Waste Package Component Exposed to Vibratory Ground Motion-The motion of repository components (namely waste package, pallet, and drip shield) due to a seismic event is evaluated using a three-dimensional finite element representation with an acceleration time history as an externally applied load. Because these repository components are not anchored to the drift invert or to each other, in case of an extremely intense seismic event they are free to move and impact each other, generating considerable contact forces as well as material and structural (geometrical) nonlinearities. The nonlinearity of the problem is further exacerbated by an essential role played by friction. The primary objective of these simulations is to evaluate structural response of the waste package outer corrosion barrier in the course of seismic events of various intensities and frequencies of occurrence. The maximum stress intensity and the residual ( $1^{\text {st }}$ principal) stress field are, therefore, determined in order to evaluate likelihood of immediate breach of the outer corrosion barrier and to estimate the area of the containment barrier in which the residual stress exceeds a certain stress threshold. This analysis is intended for use in support of the total system performance assessment (TSPA)-License Application. The simulations are performed by using the double-precision version of the explicit LS-DYNA finite element code. Multiple simulations are performed at each annual frequency of occurrence. The stochastic input parameters in these structural response calculations are the ground motion time history, metal-to-metal friction coefficient, and metal-to-rock friction coefficient. Structural response is evaluated at two different environment temperatures, and the effect of the drip shield constraint is studied to a certain extent.

Evaluation of Drip Shield Exposed to Vibratory Ground Motion-The motion of the three interlocking drip shields due to a seismic event are evaluated using a three-dimensional finite element representation with an acceleration time history as an externally applied load. The primary objective of these simulations is to evaluate structural response of the drip shield in the course of seismic events of various intensities and frequencies of occurrence. The maximum stress intensity and residual $\left(1^{\text {st }}\right.$ principal) stress field are, therefore, determined in order to evaluate likelihood of immediate breach of the drip shield plates and to estimate the drip shield area in which the residual stress exceeds a certain stress threshold. Another important goal is to examine the likelihood of drip shield separation during the seismic event. This analysis is intended for use in support of the TSPA-License Application. The simulations are performed by using the double-precision version of the explicit LS-DYNA finite element code. Multiple simulations are performed at each annual frequency of occurrence. The stochastic input 
parameters in these structural response calculations are the ground motion time history, metal-tometal friction coefficient, and metal-to-rock friction coefficient. Structural response is evaluated at two different environment temperatures.

\subsubsection{Residual Stress Reduction}

The waste package is designed to retain hermeticity for very long periods of time. The chief challenge to maintaining this objective is stress corrosion cracking. Three conditions are simultaneously required to induce stress corrosion cracking: a corrosive environment, a material susceptible to corrosion, and tensile stresses. Removing or reducing the effect of any one of these conditions could eliminate or lessen the potential for stress corrosion cracking. While a non-corrosive environment cannot be assured, Alloy 22 N06022 has been chosen as the waste package outer corrosion barrier material by virtue of its low susceptibility to corrosion, addressing the material requirement. However, residual tensile stresses in the final closure weld of the waste package may make Alloy 22 N06022 susceptible to stress corrosion cracking at these weld locations.

Two countermeasures are currently being evaluated to remove residual tensile stresses in the waste package final closure weld by inducing compressive stresses in the weld. One is controlled plasticity burnishing; the other is laser peening. A third counter measure (Induction Heating) provides an additional backup method to remove residual tensile stresses. The details of these methodologies have not been established at the time of this writing, but a brief description of each is given below. The method of choice will be determined at a future date.

Localized Compressive Stress Creation by Laser Peening-In the laser peening operation, high-energy density laser pulses with selected intensities and duration are directed at the surface of the final closure weld. A method is currently being developed to confine the release of laser pulse energy at the material surface. The resulting pressure or shock wave is propagated into the material, thus imparting a compressive surface residual stress layer in the affected material.

Localized Compressive Stress Creation by Controlled Plasticity Burnishing-Controlled plasticity burnishing is a method to apply deep compressive residual stresses to the final closure weld. Controlled plasticity burnishing produces a layer of compressive stress of high magnitude and depth, with minimal plastic deformation. In the controlled plasticity burnishing process, a smooth, free-rolling spherical ball is pressed against and rolled along the surface of the material. To ensure free rolling, the ball is supported in a fluid bearing spherical-socket with sufficient fluid pressure and flow to maintain the ball out of contact with the socket. The normal force with which the ball is pressed against the surface is made large enough to deform the surface layer of material into a state of compression, taking account of any tensile stress that exists in the material prior to burnishing.

Weld Annealing by Induction Heating-Induction heating may provide an alternative technology to induce compressive stresses. This technique involves localized treatment of the material using induction heating. The material is then quickly cooled to room temperature by quenching. At present there are no plans to use this technique. 


\subsubsection{Mesh Discretization}

The purpose of mesh refinement is to ensure the mesh objectivity of the finite element analyses, i.e., that the results obtained are not mesh-sensitive. The mesh-refinement study consists of the development of an optimum (cost-effective) mesh that is believed to give mesh-objective (mesh-insensitive) results. That mesh is then refined again and computational results for the two mesh sizes are compared. The finite-element representation is considered mesh-objective if the relative difference in results between the two meshes is approximately an order of magnitude smaller than the relative difference in mesh size in the region of interest; otherwise further mesh refinement is needed. The mesh size, as used throughout this section, refers to the volume or the area of the representative (3-dimension or 2-dimension, respectively) element in the region of interest (for example, the element characterized by the highest stresses or strains).

The optimum mesh is created by the following sequence of steps:

The initial mesh is created by pursuing the customary engineering practices: the element type is appropriately chosen; the mesh is refined in the regions of interest (the highest stress/strain regions, initial impact regions, stress concentration regions, etc.); the mesh is mapped whenever possible; and the aspect ratio of elements is kept reasonable.

In the region of interest, the initial mesh is refined in one direction while the element size in the other two directions is kept unchanged (for example, the mesh is refined across the thickness while kept unchanged in the hoop and axial directions). The mesh-refinement procedure is repeated in this manner until the relative difference in results between the two successive meshes is acceptable (i.e., approximately an order of magnitude smaller than the relative difference in the mesh size). The mesh dimension in this direction is then fixed at the largest value that satisfied the previously mentioned criterion.

The same procedure is consecutively repeated in the remaining two directions.

The intention of this one-direction-at-a-time mesh refinement is to create, in a consistent and systematic manner, a mesh that is cost-effective and objective.

Whether the created mesh meets the latter requirement is verified by the final step: the simultaneous mesh refinement in all three directions. The level of this mesh refinement should be similar in all three directions. In this final step, the same mesh-acceptance criterion is evoked: the mesh is considered objective if the relative difference in results between the two meshes is approximately an order of magnitude smaller than the relative difference in mesh size in the region of interest.

It should be emphasized that the mesh objectivity is verified by the final step regardless of whether the final mesh is arrived at by the described one-direction-at-a-time mesh refinement or not. The one-direction-at-a-time mesh refinement is optional because its only purpose is to develop a cost-effective mesh (that satisfies the objectivity requirement). 


\subsubsection{Waste Package Component Design Stress Limits and Failure Criteria}

For structural analyses of preliminary designs that consider material nonlinear behavior, the maximum-shear-stress or Tresca (strength of materials) criterion is used in determining stress limits. In general terms, this criterion assumes that the design is safe as long as stress intensity (the difference between maximum and minimum principal stress) remains below a certain limit. In particular, the failure criterion chosen was the acceptance criteria for plastic analysis (ASME 2001 [DIRS 158115] Section III, Division 1, Appendix F, F-1341.2). This is an acceptable vessel designer choice of ASME Code acceptance criteria for service loadings with Level D service limits for vessel designs in accordance with NC-3200 (Safety Class 2 vessels) when a complete stress analysis is performed (ASME 2001 [DIRS 158115], NC-3211.1(c), Appendix XIII and Note (4) to Table NC-3217-1).

The ASME Code suggests the following primary stress intensity limits for plastic analyses (ASME 2001 [DIRS 158115], Section III, Division 1, Appendix F, F-1341.2):

- The general primary membrane stress intensity shall not exceed $0.7 S_{u}$ for ferritic steel materials included in Section II, Part D, Subpart 1, Table 2A and the greater of $0.7 S_{u}$ and $S_{y}+{ }_{1}^{1} 3\left(S_{u}-S_{y}\right)$ for austenitic steel, high-nickel alloy, and copper-nickel alloy materials included in Section II, Part D, Subpart 1, Table 2A, where $S_{u}$ and $S_{y}$ are tensile strength and yield strength, respectively.

- The maximum primary stress intensity at any location shall not exceed $0.9 S_{u}$.

- The average primary shear across a section loaded in pure shear shall not exceed $0.42 S_{u}$.

The Pressure Vessel Research Council of the Welding Research Council provides guidelines (Hechmer and Hollinger 1998 [DIRS 166147]) to the ASME Boiler and Pressure Vessel Code Rule Committees for assessing stress results from three-dimensional finite element analysis in terms of stress limits in the design-by-analysis rules (ASME 2001 [DIRS 158115], Section III [Class 1, NB] and Section VIII, Division 2). These guidelines were developed for linear analyses and Pressure Vessel Research Council recommends that future research work should be conducted to generate state-of-the-art guidelines for applying inelastic, large-deformation analyses. Therefore, a cautious use of the Pressure Vessel Research Council recommendations was made in developing methodologies for post-processing LS-DYNA nonlinear plastic simulations to assure conservative representations of the general primary membrane stress intensity and maximum primary stress intensity.

The Pressure Vessel Research Council recommendations also refer to an earlier Pressure Vessel Research Council (Phase 1) report (Hechmer and Hollinger 1998 [DIRS 166147]), which recommended that the ASME Code (ASME 2001 [DIRS 158115], Appendix F) "should be revised to provide a limit on effective plastic strain which is more appropriate for events that are energy controlled, rather than load controlled, which is all that was considered when ASME B\&PV [Boiler and Pressure Vessel] Code Appendix F was written." The YMP recognizes that strain-based or deformation-based criterion may be more appropriate than stress-based limits for evaluation of the credible preclosure sequence events (Section 4.1.4.1). However, the project is also committed to applying the ASME Code for structural analyses, and until the ASME Boiler 
and Pressure Vessel Code Rule Committees prepare rules in the ASME Code, Appendix F (ASME 2001 [DIRS 158115], Appendix F) for using strain limits, primary stress intensity limits will be used.

The ASME Code design-by-analysis guidance recognizes the differences in importance of different types of stresses and provides guidance on their correct assignment to the different categories of stress intensity used to evaluate different types of failure modes (ASME 2001 [DIRS 158115]). The three types of stresses are membrane, bending and peak stresses. The three categories of stress intensity are primary $\left(P_{m}, P_{L}\right.$ and $P_{b}$ [general primary membrane, local primary membrane, and primary bending, respectively]), secondary $(Q)$, and peak $(F)$.

A primary stress is defined as "a normal stress developed by the imposed loading which is necessary to satisfy the laws of equilibrium of external and internal forces and moments. The basic characteristic of a primary stress is that it is not self-limiting. Primary stresses which considerably exceed the yield strength will result in failure or, at least, in gross distortion" (ASME 2001 [DIRS 158115], Section III, Division 1, Appendix XIII, XIII-1123(h)).

A secondary stress is defined as "a normal or a shear stress developed by the constraint of adjacent parts or by self-constraint of the structure. The basic characteristic of a secondary stress is that it is self-limiting. Local yielding and minor distortions can satisfy the conditions which cause the stress to occur and failure from one application of the stress is not expected" (ASME 2001 [DIRS 158115], Section III, Division 1, Appendix XIII, XIII-1123(i)). A cited example of a secondary stress is "bending stress at a gross structural discontinuity." A gross structural discontinuity is defined as "a source of stress or strain intensification which affects a relatively large portion of a structure and has a significant effect on the overall stress or strain pattern or on the structure as a whole" (ASME 2001 [DIRS 158115], Section III, Division 1, Appendix XIII, XIII-1123(b)). Cited examples of gross structural discontinuities are head-to-shell junctions and junctions between shells of different thickness.

A local primary membrane stress is defined as "a membrane stress produced by pressure or other mechanical loading and associated with a discontinuity [that] would, if not limited, produce excessive distortion in the transfer of load to other portions of the structure. Conservatism requires that such a stress be classified as a local primary-membrane stress even though it has some characteristics of a secondary stress" (ASME 2001 [DIRS 158115], Section III, Division 1, Appendix XIII, XIII-1123(j)). The other differentiating feature of a local primary membrane stress is that it is localized, and guidance is provided (ASME 2001 [DIRS 158115]) for evaluating if membrane stress fields are adequately "local" to be assigned a $P_{L}$ classification rather than a more restrictive $P_{m}$ classification.

The failure mode being addressed by the general primary membrane stress intensity $\left(P_{m}\right)$ limit is "collapse" in the sense that collapse includes tensile instability and ductile rupture under short term loading (Hechmer and Hollinger 1998 [DIRS 166147], Guideline 1). The principle failure mode being addressed by the maximum primary stress intensity $\left(P_{L}+P_{b}\right)$ is excessive plastic deformation. However, it also relates to tensile instability due to the nature of $P_{b}$.

The sequence events considered in this report are not repetitive where fatigue cracking or incremental collapse might be an issue. It follows that evaluation of secondary stress intensities 
(Q) or maximum total stress intensities $\left(P_{L}+P_{b}+\mathrm{Q}+\mathrm{F}\right)$ are not appropriate. Brittle fracture is also precluded by the high ductility of the outer boundary material, Alloy 22 (UNS N06022), at the temperatures experienced after waste form loading. Although the high-stress areas are comprised of primary, secondary, and peak stresses, only the primary stress intensities $\left(P_{m}, P_{L}\right.$ and $P_{b}$ ) contribute to plastic instability (tensile tearing) or excessive plastic deformation, and therefore, only the primary stress intensities are evaluated for the sequence events.

The ASME Code was used to determine which stress fields should be classified as primary and which should be classified as secondary when evaluating the sequence events (ASME 2001 [DIRS 158115], Section III, Division 1, Appendix XIII, Table XIII-1130-1). All membrane stress fields were conservatively classified as primary. Classification of the bending stresses was more involved.

Review of representative analyses for the sequence events indicated that the most important wall-bending stresses in the outer corrosion barrier occurred near gross structural discontinuities. Some of these gross structural discontinuities were integral to the outer boundary and some were introduced by the constraint of adjacent parts or impact surfaces.

The integral gross discontinuities in the outer corrosion barrier are similar to ASME Code vessel details such as shell-to-lid junctures and step-changes in wall thickness. The bending stresses are being created by self-constraint, and the ASME Code classifies these bending stresses as secondary (ASME 2001 [DIRS 158115], Section III, Division 1, Appendix XIII, Table XIII1130-1). The only exception is at the shell-lid junction, where concern about the predictability of the central stresses of the lid leads the ASME Code to caution the designer to consider classifying the bending stresses as $P_{b}$ (ASME 2001 [DIRS 158115], Section III, Division 1, Appendix XIII, Table XIII-1130-1, Note (4)). However, this is not appropriate guidance for inelastic analyses because the increased flexibility of the juncture caused by inelastic behavior is correctly captured and the central stresses of the lid are accurately predicted.

The bending stresses created by the constraint of adjacent parts or impact surfaces (which can be considered [temporary] "adjacent parts") were reviewed on individual cases with attention to the amount and type of constraint introduced. In the design analyses to date, the constraint of the adjacent part (e.g., trunnion sleeve) or impact surface (e.g., emplacement pallet, crane hook, or rock) created local yielding and minor distortions in the outer barrier. The outer corrosion barrier distorted shape reduced the outer corrosion barrier bending stresses while increasing the outer corrosion barrier membrane stresses. The bending stresses in these locally yielded regions are therefore self-limiting and satisfy the basic characteristic of a secondary stress.

The structural criterion developed for the outer boundary for the sequence events was to directly address the dominant failure mode, tensile instability, and limit the membrane stresses to acceptable limits. The use of inelastic analyses ensures that local thinning or shape changes that could increase membrane stresses are properly accounted for.

Inelastic analyses were conducted using true stress and true strain based constitutive relationships, therefore for Alloy 22 (UNS N06022), the limit on $P_{m}$ is $0.7 \sigma_{\mathrm{u}}$, the limit on $P_{L}$ is $0.9 \sigma_{u}$ (where $P_{b}=0$ ), where $\sigma_{u}$ is the true tensile strength at temperature (ASME 2001 [DIRS 158115], Section III, Division 1, Appendix F, F-1322.3(b) and F-1341.2). 
As stated earlier, $P_{L}$ must be "local" to not be classified as a more restrictive general primary membrane stress intensity, $P_{m}$ (ASME 2001 [DIRS 158115], Section III, Division 1, Appendix XIII, XIII-1123(j)). Interpretation of this guidance with respect to the ASME B\&PV Code Appendix F (ASME 2001 [DIRS 158115]) limits results in requiring $P_{L}$ values exceeding $0.77 \sigma_{u}$ to not extend for greater than $\sqrt{R \cdot t}$ in any direction, where $R$ is the midsurface radius and $t$ is the thickness of the outer barrier.

Rigorously performed, calculation of the primary membrane stress intensities involves:

- Identifying the governing wall location, which may not necessarily contain the maximum stressed point (Hechmer and Hollinger 1998 [DIRS 166147], Guidelines 3 and 4)

- Identifying the orientation of the stress classification line, typically normal to the midplane of the shell or lid thickness (Hechmer and Hollinger 1998 [DIRS 166147], Guideline 4d).

- Identifying the stress component $\left(\sigma_{x}, \sigma_{y}, \sigma_{z}, \tau_{x y}, \tau_{y z}, \tau_{z x}\right)$ fields across the wall of the outer corrosion barrier

- Averaging the stress component fields to create wall-averaged stress components

- Translating the wall-averaged stresses to principle stress directions by solving a cubic equation

- Calculating the difference between the maximum $\left(\sigma_{1}\right)$ and minimum $\left(\sigma_{3}\right)$ principle stress direction values.

To simplify the calculation, the wall-average of the element total stress intensity (twice the maximum shear stress) values through the outer corrosion barrier is used to define the primary membrane stress intensities. This is a conservative representation because it ignores possibly changing principle stress planes through the wall, and it includes the secondary and peak stress contributions.

The failure criterion used is broken into tiered screening criteria shown below. The easiest to apply and most conservative criteria are applied initially. If these can not be met, less conservative screening criteria are imposed that require more calculations. These screening criteria in decreasing order of conservatism are listed below. An element's total stress intensity, $\sigma_{\text {int }}$, is equal to twice the element's maximum shear stress (ASME 2001 [DIRS 158115], Section III, Division 1, NB-3000).

\begin{tabular}{|llr|l|}
\hline \multicolumn{2}{|c|}{ Criteria } & \multicolumn{1}{c|}{ Condition of Acceptance } \\
\hline $\begin{array}{l}\text { Maximum } \\
\text { No. }\end{array}$ & $\sigma_{\text {int }}<0.7 \sigma_{\mathrm{u}}$ ? & $\begin{array}{l}\text { Yes: Meets } P_{m} \text { and } P_{L} \text { limits without the need for } \\
\text { wall averaging. }\end{array}$ \\
\hline $\begin{array}{l}\text { Maximum } \\
\text { No. }\end{array}$ & $\sigma_{\text {int }}<0.77 \sigma_{\mathrm{u}}$ ? & $\begin{array}{l}\text { Yes: Meets } P_{L} \text { limit without the need for wall } \\
\text { averaging, but the stress field must not be uniform }\end{array}$ \\
\hline
\end{tabular}




\begin{tabular}{|l|l|}
\hline & $\begin{array}{l}\text { around the entire circumference (only a concern } \\
\text { for vertical drop events). }\end{array}$ \\
\hline $\begin{array}{l}\text { Maximum wall-averaged } \sigma_{\mathrm{int}}<0.7 \sigma_{\mathrm{u}} ? \\
\text { No. }\end{array}$ & Yes: Meets $P_{m}$ and $P_{L}$ limits. \\
\hline $\begin{array}{l}\text { Maximum wall-averaged } \sigma_{\mathrm{int}}<0.77 \sigma_{\mathrm{u}} ? \\
\text { No. }\end{array}$ & $\begin{array}{l}\text { Yes: Meets } P_{L} \text { limit if the stress fields are not } \\
\text { uniform around the entire circumference (only a } \\
\text { concern for vertical drop events). }\end{array}$ \\
\hline $\begin{array}{l}\text { Maximum wall-averaged } \sigma_{\mathrm{int}}<0.84 \sigma_{\mathrm{u}} \\
\text { and wall-averaged } \sigma_{\text {int }}<0.77 \sigma_{\mathrm{u}} \text { at } \sqrt{R \cdot t} \\
\text { surrounding maximum location? } \\
\text { No. }\end{array}$ & Yes: Meets $P_{L}$ and average primary shear limit. \\
\hline $\begin{array}{l}\text { Maximum wall-averaged } \sigma_{\mathrm{int}}<0.9 \sigma_{\mathrm{u}} \\
\text { and wall-averaged } \sigma_{\text {int }}<0.77 \sigma_{\mathrm{u}} \text { at } \\
\sqrt{R \cdot t} \text { surrounding maximum location } \\
\text { and wall-average of each shear stress on } \\
\text { the stress classification line }\left(\tau_{\mathrm{xy}}, \tau_{\mathrm{yz}} \text { and }\right. \\
\left.\tau_{\mathrm{xz}}\right)<0.42 \sigma_{\mathrm{u}} ?(\mathrm{x}, \mathrm{y}, \mathrm{z} \text { are element (not } \\
\text { global) directions) } \\
\text { No: Fails simplified screening criterion. }\end{array}$ & $\begin{array}{l}\text { Yes: Meets } P_{L} \text { and average primary shear limit } \\
\text { orthogonal to the Stress Classification Line) }\end{array}$ \\
\hline
\end{tabular}

Note: $P_{m}$ is the general primary membrane stress intensity

$P_{L}$ is the local primary membrane stress intensity

$P_{b}$ is the primary bending stress intensity

$R$ is the median wall radius

$t$ is the wall thickness.

If the wall-averaged $\sigma_{\text {int }}$ limits can not be met, perform a more rigorous evaluation using all six stress components (and solve a cubic equation for principle stress direction values) or use multiple stress classification lines to extrapolate to governing wall locations when they have significant non-membrane stress contributions.

If the average primary shear limit can not be met, then review appropriateness of using a stress classification plane rather than a stress classification line.

If the screening criteria can not be met, perform a rigorous Code evaluation using quantitative instead of bounding stress classifications. This will require additional elastic finite element analysis with variable Modulus of Elasticity and time-slicing.

For lifting analyses, the acceptance criteria are outlined in American National Standard for Radioactive Materials-Special Lifting Devices for Shipping Containers Weighing 10000 Pounds (4500 kg) or More (ANSI N14.6-1993 [DIRS 102016], Section 4.2.1.1). The loadbearing members of the lifting device shall be capable of lifting three times the combined weight 
of the shipping container, plus the weight of the intervening components of the lifting device, without generating a combined shear stress or maximum principal stress at any point in the device in excess of $S_{y}$. The lifting device shall also be capable of lifting five times the weight without exceeding $S_{\mathrm{u}}$.

Finally, for structural analyses of the seismic events, the acceptance criterion is based on stresscorrosion-cracking considerations. It is generally assumed that crack initiation will not occur if the residual stress is below a certain threshold value. Thus, the first residual principal stress at any location exposed to an aggressive environment shall not exceed the lower bound of $0.8 S_{y}$ as suggested by Errata for Seismic Consequence Abstraction (BSC 2004 [DIRS 167780], Section 6.3.1, p. 32).

\subsubsection{Calculations for True Measures of Ductility}

The material properties in the engineering handbooks and vendor catalogs refer to engineering stress and strain definitions: $s=P / A_{0}$ and $e=L / L_{0}-1$ (Dieter 1976 [DIRS 118647], Chapter 9), where $P$ is the force applied during a static tensile test, $L$ is the length of the deformed specimen, and $L_{0}$ and $A_{0}$ are the original length and cross-sectional area of the specimen, respectively. The engineering stress-strain curve does not give a true indication of the deformation characteristics of a material during plastic deformation because it is based entirely on the original dimensions of the specimen. In addition, ductile metal that is pulled in tension becomes unstable and necks down in the course of the test. Hence, LS-DYNA finite element code requires input in terms of true stress and strain definitions: $\sigma=P / A$ and $\varepsilon=\ln \left(L / L_{0}\right)$.

The true stresses and strains $(\sigma, \varepsilon)$ are calculated by using their engineering counterparts $(s, e)$ based on the following relations:

$$
\begin{gathered}
\sigma=s \cdot(1+e) \\
\varepsilon=\ln (1+e)
\end{gathered}
$$

Equations 1 and 2 can be readily derived based on constancy of volume $\left(A_{0} \cdot L_{0}=A \cdot L\right)$ and strain homogeneity during plastic deformation (Dieter 1976 [DIRS 118647], Chapter 9). These expressions are applicable only in the hardening region of the stress-strain curve that is limited by the onset of necking.

Equations 1 and 2 are used to calculate true tensile strength and true uniform strain (the strain corresponding to tensile strength). These material properties are then used to calculate the hardening (tangent) modulus (Figure 9). 


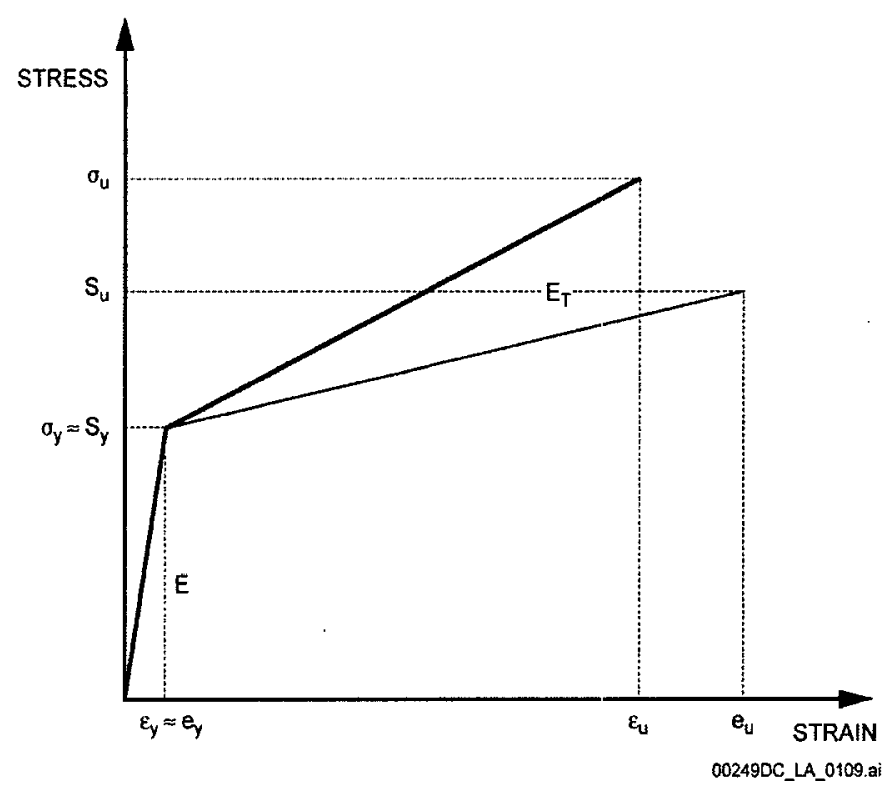

Figure 9. Key Attributes of Bilinear Stress-Strain Curves

\subsubsection{Fracture Mechanics Analyses}

The structural analysis methods discussed so far predict margin to failure by ductile collapse. Fracture Mechanics may be used to analyze the potential for brittle fracture. Depending on the material properties, a combination of ductile and brittle failure may need to be considered. The waste package and the drip shield materials, in general, possess ductile behavior. Specific material properties for specific structural calculations may show that only ductile collapse must be considered. For such cases, no further investigation of brittle fracture is required. However, any potential brittle behavior due to environmental conditions in the repository, such as the hydrogen embrittlement of titanium or fabrication effects/defects on titanium and Alloy 22 N06022 may require further investigation of these materials. For these cases, fracture mechanics calculations can be performed using either the stress intensity factor or the J-integral at the crack tip, depending on the extent of plasticity that results from the impact. If these fracture parameters exceed the material limits, the crack propagation will be evaluated. Otherwise, the crack growth is arrested and there is no failure. Hence, a rigorous analysis of the crack propagation scheme may determine the consequence of such an event.

The specific problem of crack propagation in the waste package and drip shield materials involves low-velocity impact of two structural components. Existing cracks (manufacturing flaws) on metallic plates may be analyzed. The crack propagation or arrest under dynamic loads due to rock impacts or handling accidents may be investigated using the commercially available software. This problem may require elastic-plastic material properties and large deformation simulations in addition to the contact between the impacting object and the metal plate.

\subsection{THERMAL DESIGN}

The purpose of the waste package thermal analyses is to ensure that the waste form temperatures do not exceed levels that are important to maintaining their long-term integrity. For commercial 
SNF, this involves ensuring that the cladding temperature does not induce rupture, compromising the cladding as a barrier to radionuclide release. For DOE SNF, thermal limits will be defined. For defense HLW glass, this involves ensuring that the glass does not reach a transition temperature that would result in an alteration of the glass microstructure, increasing the solubility of the glass and reducing the time required for mobilization of the radionuclides embedded in the glass matrix.

Various thermal calculations require different computational tools and may be performed for different levels of detail and accuracy. The suite of computational tools used is discussed in Section 6.3.1, and the types of calculations performed for thermal analysis are discussed in Section 6.3.2.

\subsubsection{Computational Tools}

Thermal analyses are performed to assure that fuel cladding, waste package, and rock temperatures do not exceed maximum limits. Benchmarking calculations are performed to demonstrate that the thermal analysis methods produce valid results. The benchmark calculations will include comparisons with data from large experiments at Yucca Mountain, including the Large Block Test, the Single Heater Test, and the Drift Scale Test. Benchmark calculations of forced and natural convection are compared to data from quarter scale testing.

\subsubsection{ANSYS}

The primary computational tool used for heat transfer calculations is ANSYS. ANSYS solves all three modes of heat transfer: conduction, convection, and radiation. Typically, convection is not specifically calculated in ANSYS. Instead, heat removed by ventilation is subtracted from the total heat source and only the net heat transferred to the drift walls is included in the calculations. For conduction, the thermal conductivity and specific heats may be spatially varying and temperature-dependent. For radiation, ANSYS determines an effective thermal conductivity and applies it to Fourier's Law of heat conduction. This effective thermal conductivity is computed from the thermal communication between each element of the surfaces, using gray body diffuse radiation theory. For an enclosed system of finite radiating surfaces, the theory of gray body diffuse radiation heat transfer is appropriate (Siegel and Howell 1992 [DIRS 100687], Equation 7-31, p. 271).

ANSYS allows three types of thermal boundary conditions: temperature, heat flux, and a convection condition. The boundary condition of convection does not imply a detailed convection calculation but rather a heat flux proportional to the difference between the instantaneous surface temperature and the free-stream temperature (i.e., Newton's Law of Cooling). These boundary conditions are applied at the surfaces of the problem domain.

Consistent with finite element analysis, the problem domain is divided into polygons. Within these solids, the thermal transport properties and volumetric heat generation magnitudes (as appropriate) are spatially constant. However, the variation in temperature within and among the polygons is approximated by one of a number of shape functions. This technique permits larger polygons to be used than would be possible with uniform temperatures within the polygons and a simplistic relationship among the polygons. 


\subsubsection{FLUENT}

The FLUENT computational fluid dynamics code is used to calculate heat transfer coefficients, air temperature, air velocity, heat flux, and pressure distribution in the drift.

\subsubsection{DRIFTFLOW}

DRIFTFLOW is a Microsoft Visual Basic macro that operates in Microsoft Excel 97. DRIFTFLOW is intended to represent the entire repository and give a quick best-estimate of thermal conditions therein, during preclosure (forced ventilation as well as natural ventilation) and postclosure periods. Conduction is calculated by superimposing thermal responses to a series of heat pulses calculated in ANSYS. Convection and radiation heat transfer are calculated using empirical correlations derived for a concentric tube annulus. Ventilation flow rate is specified during the forced ventilation period and calculated from pneumatic pressure differences during the natural ventilation period.

\subsubsection{MATHCAD}

MATHCAD can solve systems of equations, allowing the user to evaluate the impact of parameter variance quickly.

\subsubsection{Description of Pertinent Analyses}

Table 5 gives an outline of the various types of thermal design calculations performed.

\subsubsection{Fuel Assembly-Scale Calculations}

Fuel assembly-scale calculations are used to develop effective thermal conductivity correlations. These calculations impose a uniform surface temperature on the four sides of a fuel assembly and calculate the steady state temperature distribution. The results are used to determine effective thermal conductivity for the fuel assembly.

Effective thermal conductivity is used to predict peak cladding temperatures for SNF assemblies. Rather than representing the waste package and every fuel rod in every assembly, this method represents the fuel assemblies as a smeared (distributed) solid volume with uniform volumetric heat generation. The smeared properties represent the combined thermal radiation and conduction heat transfer from the fuel rods to the inner-basket structure. This method, developed in Spent Nuclear Fuel Effective Thermal Conductivity Report (CRWMS M\&O 1996 [DIRS 100215]), provides a best estimate of peak cladding temperatures compared to correlations such as "Wooton-Epstein" (Wooton and Epstein 1963 [DIRS 144379]), which produces an undetermined degree of conservatism. Such conservatism often leads to over-design.

To determine the appropriate effective thermal conductivities for PWR and BWR commercial SNF assemblies, detailed thermal representations of typical fuel assemblies are developed using a finite-element computer code (Figure 10). Vacuum conditions and fill gases of helium, nitrogen, and argon are evaluated with various rod array. For BWR fuel, the evaluation considers the effects of channels between fuel assemblies, cladding oxidation, locations of guide tubes and water rods, emissivity variation, and inner-basket temperature gradients. Calculated 
effective thermal conductivities are found to be highly temperature-dependent due to the contribution of thermal radiation with little dependence on assembly heat output.

Results from this method are compared to those from previous applications using alternate methods and to actual test results from spent-fuel storage casks (CRWMS M\&O 1996 [DIRS 100215], Sections 7.2 and 7.3). The comparisons to experimental data and test calculations indicate that the effective thermal conductivities provide a best estimate of cladding temperatures within a spent-fuel waste package. Further, the effective thermal conductivities are consistent with single-point values previously published by storage cask vendors (CRWMS M\&O 1996 [DIRS 100215], Section 8.0). 
Table 5. Summary of Thermal Calculations for Waste Package Component Design

\begin{tabular}{|c|c|c|c|}
\hline Type of Calculation & Phenomena Calculated & $\begin{array}{l}\text { Boundary conditions } \\
\text { Initial conditions } \\
\text { Assumptions }\end{array}$ & Calculation Results \\
\hline $\begin{array}{l}\text { 2-D ANSYS calculation at } \\
\text { Fuel Assembly-Scale }\end{array}$ & $\begin{array}{l}\text { Steady State } \\
\text { Conduction heat transfer } \\
\text { Radiative heat transfer }\end{array}$ & $\begin{array}{l}\text { Uniform assembly outer } \\
\text { temperature } \\
\text { Matrix of calculations (power, } \\
\text { temp) } \\
\text { Ignores convection heat transfer }\end{array}$ & $\begin{array}{l}\text { Results used to determine an effective } \\
\text { thermal conductivity for fuel assembly } \\
\text { as a function of temperature. Variation } \\
\text { with power is small, but averaged in } \\
\text { result. }\end{array}$ \\
\hline $\begin{array}{l}\text { 2-D ANSYS calculation at } \\
\text { Waste Package-Scale }\end{array}$ & $\begin{array}{l}\text { Pseudo-Transient } \\
\text { Conduction heat transfer } \\
\text { Radiation heat transfer }\end{array}$ & $\begin{array}{l}\text { Waste package temperatures } \\
\text { specified at top, side and bottom, } \\
\text { and interpolated between these } \\
\mathrm{K}_{\mathrm{eft}} \text { for each assembly } \\
\text { lgnores convection heat transfer }\end{array}$ & $\begin{array}{l}\text { Keff for entire waste package contents } \\
\text { Waste package interior temperature } \\
\text { distribution (most importantly the peak } \\
\text { cladding temperature) vs. time. }\end{array}$ \\
\hline $\begin{array}{l}\text { 2-D or 3-D ANSYS calculation at } \\
\text { Drift-Scale }\end{array}$ & $\begin{array}{l}\text { Pseudo-Transient } \\
\text { Conduction and radiation heat } \\
\text { transfer }\end{array}$ & $\begin{array}{l}\text { Uniform temp } 5 \mathrm{~m} \text { into rock wall } \\
\text { obtained from "pillar" calculation at } \\
\text { same linear heat rate } \\
\text { Keff }_{\text {er waste package contents }}\end{array}$ & $\begin{array}{l}\text { Evaluate impact of drip shield and invert } \\
\text { on waste package surface and peak } \\
\text { cladding temperatures vs. time. }\end{array}$ \\
\hline $\begin{array}{l}\text { 2-D ANSYS calculation at } \\
\text { Repository Scale } \\
\text { ("pillar".calculation) }\end{array}$ & $\begin{array}{l}\text { Transient } \\
\text { Conduction and radiation heat } \\
\text { transfer }\end{array}$ & $\begin{array}{l}\text { Lower and upper sink temps. } \\
\text { Line heat source } \\
\text { Keff for waste package contents } \\
\text { Arbitrary deletion of heat removed } \\
\text { by ventilation }\end{array}$ & $\begin{array}{l}\text { Response surface for peak drift wall } \\
\text { temperatures vs. repository parameters } \\
\text { such as ventilation duration and linear } \\
\text { heat loading. Each peak drift wall (or } \\
5 \mathrm{~m} \text {-into-rock) temperature on the } \\
\text { response surface can then be used as a } \\
\text { boundary condition for a drift-scale. } \\
\text { calculation, to also generate such a } \\
\text { response surface for peak waste } \\
\text { package surface temperature. }\end{array}$ \\
\hline $\begin{array}{l}\text { Axisymmetric ANSYS calculation } \\
\text { for Fire Analysis }\end{array}$ & $\begin{array}{l}\text { Transient } \\
\text { Radiation and convection from } \\
\text { fire, only radiation cooling after } \\
\text { fire }\end{array}$ & $\begin{array}{l}\text { Stainless steel initial temp } \\
\text { Detailed waste package contents }\end{array}$ & $\begin{array}{l}\text { Waste package surface, peak shell, and } \\
\text { peak cladding temperatures }\end{array}$ \\
\hline $\begin{array}{l}\text { Axisymmetric ANSYS calculation in } \\
\text { Surface Facility }\end{array}$ & $\begin{array}{l}\text { Transient } \\
\text { Conduction heat transfer } \\
\text { Radiation heat transfer }\end{array}$ & $\begin{array}{l}\text { Stainless steel initial temp } \\
\text { Ambient concrete wall temp } \\
\mathrm{K}_{\text {eff }} \text { for waste package contents }\end{array}$ & $\begin{array}{l}\text { Waste package surface, peak shell, and } \\
\text { peak cladding temperatures } \\
\text { Temperature in weld zone }\end{array}$ \\
\hline
\end{tabular}


Table 5. Summary of Thermal Calculations for Waste Package Component Design (Continued)

\begin{tabular}{|c|c|c|c|}
\hline Type of Calculation & Phenomena Calculated & $\begin{array}{l}\text { Boundary conditions } \\
\text { Initial conditions } \\
\text { Assumptions }\end{array}$ & Calculation Results \\
\hline $\begin{array}{l}\text { 1-D DRIFTFLOW calculation at } \\
\text { Repository-Scale }\end{array}$ & $\begin{array}{l}\text { Transient conduction, } \\
\text { convection, and radiation heat } \\
\text { transfer } \\
\text { Natural ventilation air flow }\end{array}$ & $\begin{array}{l}\text { Line heat source } \\
\text { Coupled with 2-D ANSYS Repository } \\
\text { Scale calculation to account for } \\
\text { transient effects of heat storage in the } \\
\text { rock. } \\
\text { Overall convective coefficient (h) from } \\
\text { FLUENT } \\
\text { Static pressure difference for natural } \\
\text { ventilation -or - flow rate for forced } \\
\text { ventilation }\end{array}$ & $\begin{array}{l}\text { Results used to determine the } \\
\text { fraction of heat removed by } \\
\text { ventilation, or ventilation } \\
\text { efficiency. } \\
\text { Waste package and drift wall } \\
\text { temperatures vs. time. }\end{array}$ \\
\hline $\begin{array}{l}\text { 3-D FLUENT calculation at Drift- } \\
\text { Scale }\end{array}$ & $\begin{array}{l}\text { Transient and steady state } \\
\text { convection, conduction, and } \\
\text { radiation heat transfer. } \\
\text { Steady state, laminar and } \\
\text { turbulent flow }\end{array}$ & $\begin{array}{l}\text { Uniform rock temperature } 5 \mathrm{~m} \text { from } \\
\text { surface (from ANSYS Repository-Scale } \\
\text { calculations) } \\
\text { Waste package surface heat flux } \\
\text { Ventilation flow rate }\end{array}$ & $\begin{array}{l}\text { Overall convective coefficient (h), } \\
\text { detailed } h \text {, } \\
\text { Waste package and drift wall } \\
\text { temperatures } \\
\text { Iterate with ANSYS to converge } \\
\text { on temperatures }\end{array}$ \\
\hline $\begin{array}{l}\text { 3-D ANSYS calculation at } \\
\text { Repository-Scale } \\
\text { ("pillar" calculation) }\end{array}$ & $\begin{array}{l}\text { Transient } \\
\text { Conduction and radiation heat } \\
\text { transfer }\end{array}$ & $\begin{array}{l}\text { Lower and upper sink temps. } \\
\text { Ketf }_{\text {ef }} \text { for waste package contents } \\
\text { Typ. Set of waste packages } \\
\text { Arbitrary deletion of heat removed by } \\
\text { ventilation }\end{array}$ & $\begin{array}{l}\text { Reference point to quantify axial } \\
\text { drift wall and waste package } \\
\text { surface temperature variations } \\
\text { along the drift, relative to the 2-D } \\
\text { repository-scale calculation. The } \\
\text { appropriate "offset" can be } \\
\text { applied to the entire response } \\
\text { surface generated from the 2-D } \\
\text { results. }\end{array}$ \\
\hline
\end{tabular}

NOTE: Transient effects for fuel assembly-scale, waste package-scale, and drift-scale calculations (out to $5 \mathrm{~m}$-into-rock) have small time constants compared to their inputs (decay of the fuel heat and mountain heat storage). Therefore, these calculations are performed as a series of steady-state calculations, at "snapshots" in time, using (a) the power produced by the fuel assemblies at that point in time, or (b) the $5 \mathrm{~m}$-into-rock temperature at that point in time.

$1-D=$ one-dimensional; $2-D=$ two-dimensional; $3-D=$ three-dimensional. 


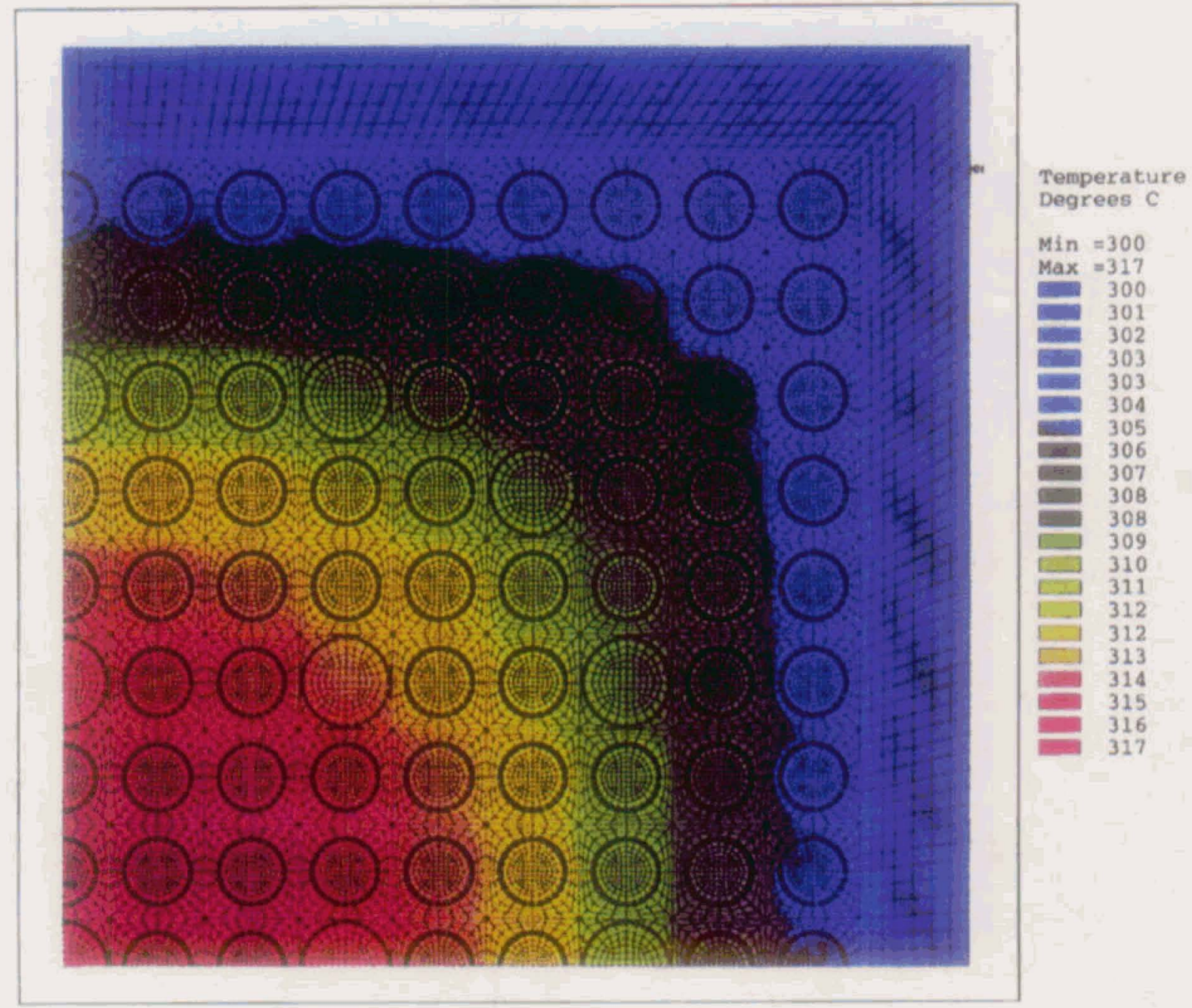

Figure 10. Finite Element Representation of Fuel Assembly

\subsubsection{Waste Package-Scale Calculations}

Thermal detail internal to a waste package is obtained using an ANSYS 2-D representation at the mid-plane of a waste package. Temperatures are imposed on the waste package surface at the top, side, and bottom positions. Interpolation is used to specify surface temperatures between these points. Fuel assemblies are represented by effective thermal conductivity, but detailed geometry of the other waste package internals is used. Waste package-scale calculations provide a means to analyze loading variations within a waste package. An effective thermal conductivity for the entire contents of a waste package can also be determined.

\subsubsection{Drift-Scale Calculations}

Drift-scale calculations can be either 2-D or 3-D but represent only the first 5 meters of rock from the drift wall. Previous calculations have shown that drift internal structures have little impact on rock temperatures at this location (CRWMS M\&O 1997 [DIRS 101538], Appendix A). Temperatures at 5-m are specified from "pillar" calculations. Calculations performed at drift-scale must use the same average heat load that was used in the corresponding 
"pillar" calculation for consistency. Drift-scale calculations provide a faster method to study variations in invert design, drip shield design, and waste package emplacement order.

\subsubsection{Repository-Scale Two-Dimensional Calculations}

While detailed repository-scale three-dimensional calculations are necessary to demonstrate margin to the waste form thermal requirements, repository-scale two-dimensional calculations are appropriate to study the sensitivity of the temperature field to changes in the major thermal variables. Such a representation consists of a perpendicular slice through a single waste package, extending from the top of the mountain to well into the saturated zone, and accounting for the thermal transport properties of each stratigraphic unit (Figure 11). Such a representation appropriately calculates the temperature field for a drift located near the center of the repository, provided the packages may be approximated as an infinitely long cylinder with an axially uniform heat generation rate.

Two-dimensional representations have low computational requirements, and a large number of calculations may be performed quickly. This rapidity of computation enables time-dependent temperature field calculations that span the design space (i.e., the range of independent variables). Low-order, multi-variant regressions may then be performed and response surfaces created. The functional form of the response surface selected is based on insight into the heattransfer physics and the fidelity with which the particular functional form reproduces the calculational results.

This representation is truly applicable only to an infinitely long waste package; however, simple adjustments may be made to approximate three-dimensional effects. The effect of increases in waste package separation is obtained by adjusting the average waste package heat generation rate (and hence the linear power). This functional form is shown in Equation 3.

$$
\mathrm{P}_{\text {linear }}=\frac{\mathrm{Q}_{\mathrm{WP}}}{\left(\overline{\mathrm{L}}_{\mathrm{WP}}+\delta\right)}
$$

Here $Q_{W P}$ is the waste package heat generation rate at emplacement, $\overline{\mathrm{L}}_{\mathrm{WP}}$ is the average-waste package length, and $\delta$ is the skirt-to-skirt gap between waste packages. The appropriateness of this adjustment decreases with increasing waste package spacing because the localized relatively high heat regions of such an arrangement are not accounted for. For the range of waste package spacings currently considered, this adjustment is applicable. 


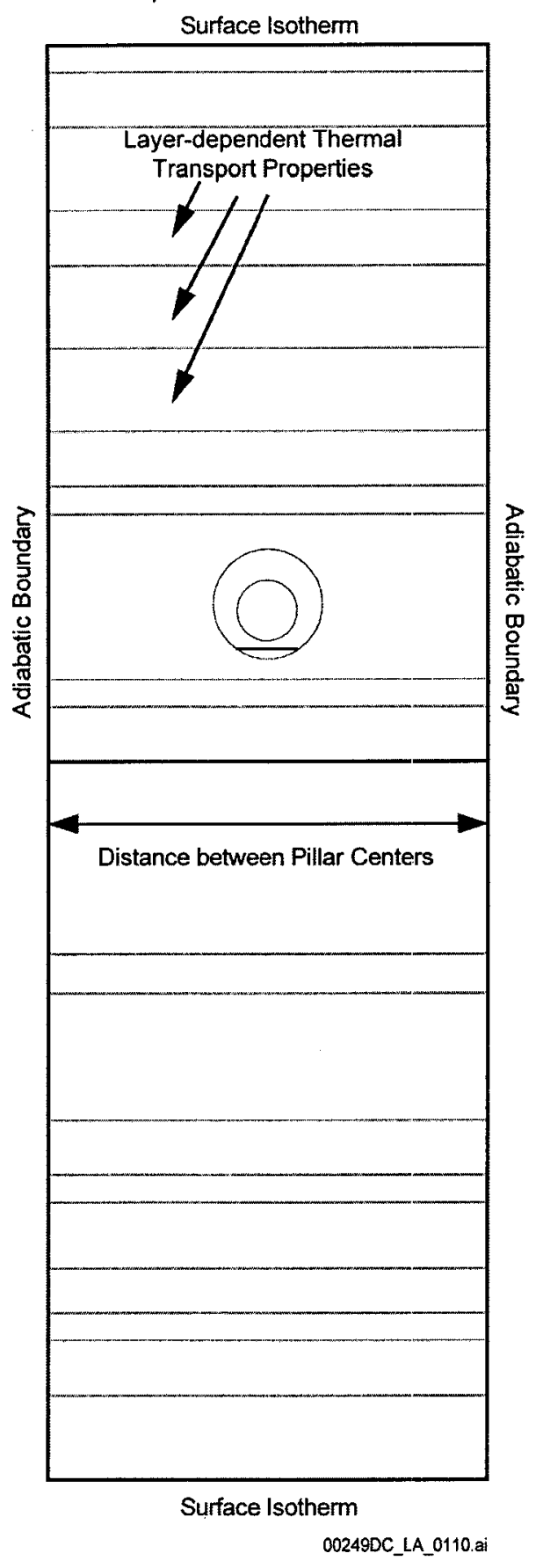

Figure 11. Illustration of Two-dimensional Repository Representation

An operating curve is the locus of values for two independent variables-holding other independent variables constant-which results in a particular temperature value on the response surface. For instance, if the ventilation duration, waste package heat generation rate at emplacement, and backfill effective-thermal conductivity (if backfill is present) are fixed, a curve may be constructed providing the combinations of skirt-to-skirt separations and heat removal fractions necessary to obtain a given peak-drift wall temperature. This process is illustrated in Figure 12. 
While these two-dimensional calculations cannot legitimately be used to quantify the effect of non-uniform heat generation rates, the results from previous three-dimensional cases may be used in conjunction with these to estimate the magnitudes of three-dimensions and nonuniformity. By assuming the nominal heat generation rate for the repository, peaking factors may be developed for a range of design basis heat generation rates. For instance, simple linear correlations for incremental temperature increases for non-uniformity may be developed. Such a functional form is shown in Equation 4.

$$
\Delta T=a_{0}+a_{1} \cdot P_{\text {linear }}
$$

Here, $a_{0}$ and $a_{1}$ are fit coefficients and $P_{\text {linear }}$ is that shown in Equation 3.

Such an expression is used to adjust upward the peak waste package-surface temperature and the corresponding peak-cladding temperature for the particular design basis heat generation rate. The waste form limit may then be decremented by the difference between the peak-cladding and waste package surface from the two-dimensional calculation. The resulting waste package surface temperature is now the target peak waste package-surface temperature. This is illustrated in Figure 13.

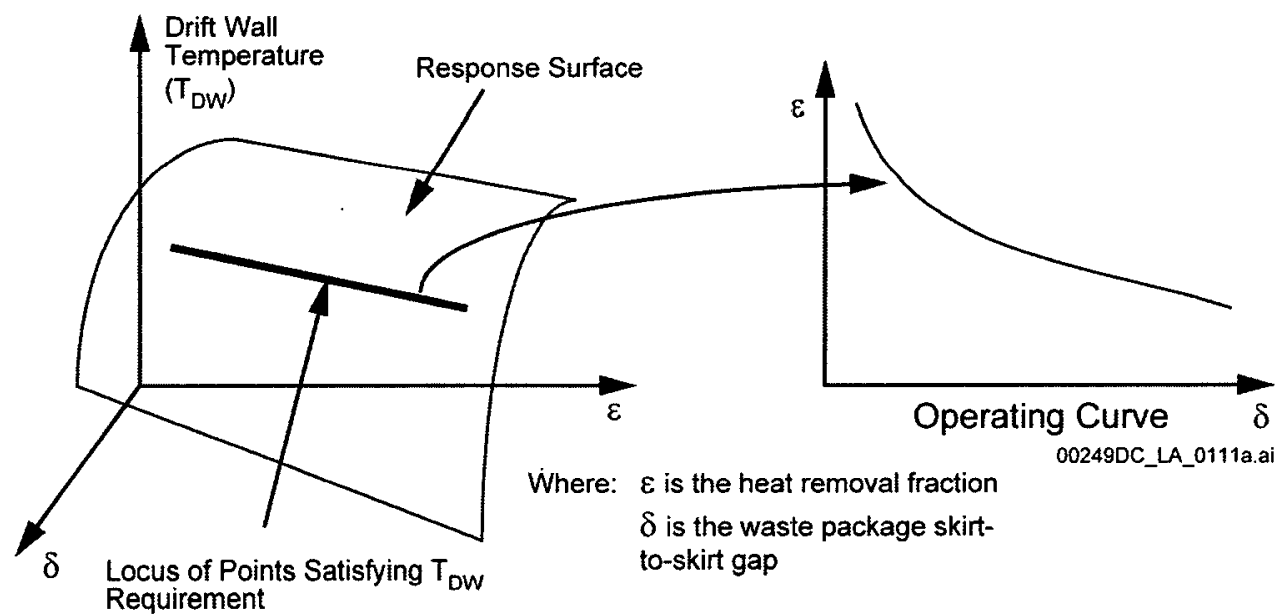

Figure 12. Illustration of Response Surface Interrogation 


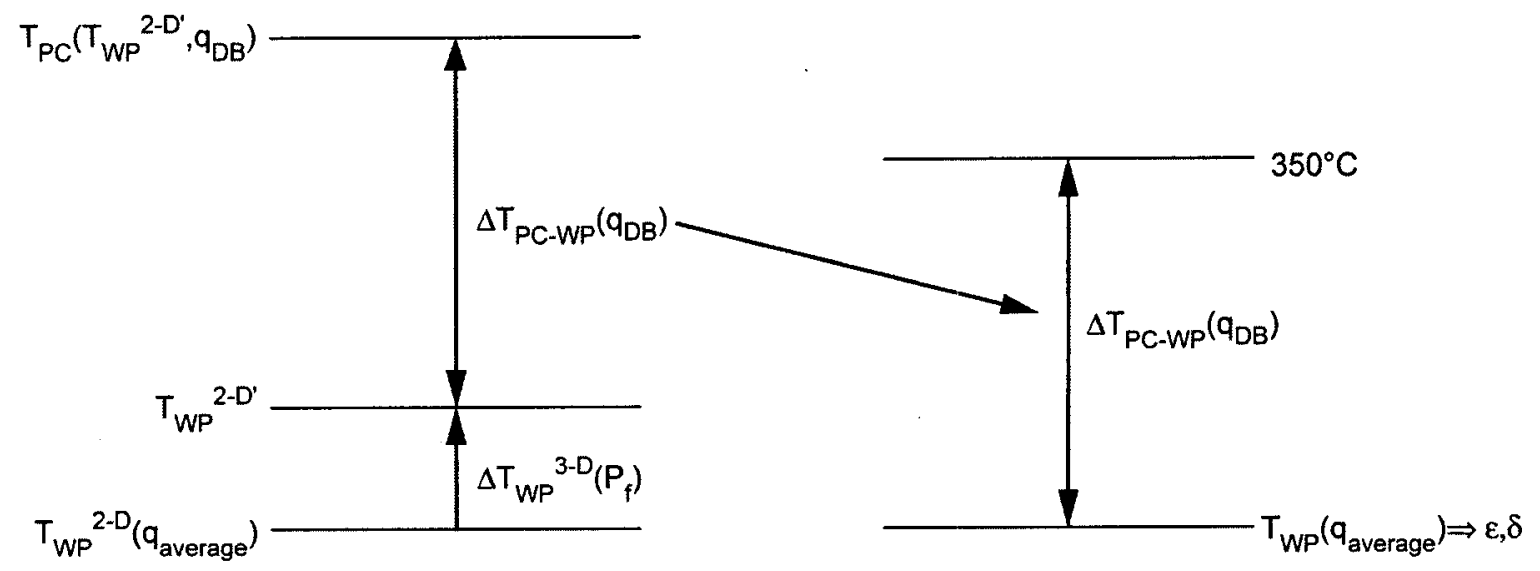

$T_{W P}^{2-D}\left(q_{\text {average }}\right)-$ WP surface temperature from response surface at the emplacement repository thermal load

$T_{W P}^{2-D^{\prime}}-$ WP surface temperature modified for three-dimensional effects

$T_{P C}\left(T_{W P}{ }^{2-D^{\prime}}, q_{D B}\right)$ - Peak-cladding temperature given modified WP surface temperature and design-basis thermal load 00249DC_LA_0112.ai

NOTE: In this process, the locus of points $\left(\varepsilon, \delta\right.$. for which $T_{P C}\left(T_{W P}{ }^{2 \cdot D^{\prime}}, q_{D B}\right)=350{ }^{\circ} \mathrm{C}$ (Minwalla 2003 [DIRS 161362], Section 5.1.3.2), is determined. This creates the operation curve for peak waste package cladding temperature.

Figure 13. Three-Dimensional Effect Accommodation

\subsubsection{Repository-Scale Three-Dimensional Analyses}

While two-dimensional calculations are appropriate for scoping studies, three-dimensional calculations are needed for detailed, final design evaluations. The interface flow of two-dimensional and three-dimensional calculations is presented in Figure 14. 


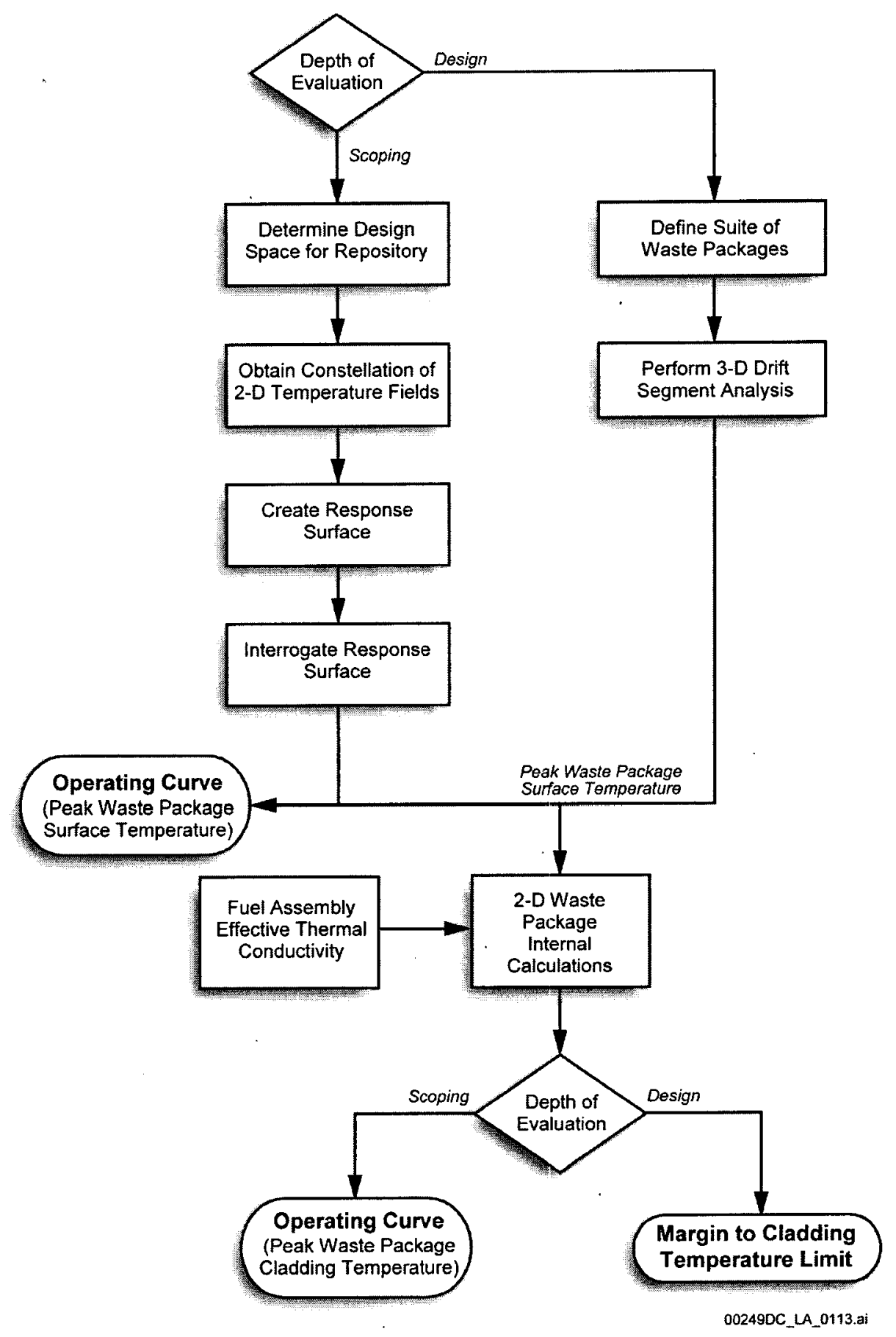

Figure 14. Thermal Analysis Technique Decision Flowchart

The thermal three-dimensional representation approximates the repository as an infinitely repeating series of "pillars," extending from the top of the mountain to a plane well into the saturated zone. Layers corresponding to the stratigraphy of the mountain represent the host rock of the repository. For each of these layers, thermal transport properties (viz., temperaturedependent thermal conductivity and specific heat) appropriate to the local rock properties are used. Laterally, adiabatic surfaces are placed at the center of the rock masses between the drifts. 
The variability of the waste package heat generation rates is incorporated by representing just a few waste packages within the drift segment. The thermal transport properties of these waste packages are represented by temperature-dependent effective values, but not with an explicit representation of the internals. The time-dependent heat generation rates of the waste packages are adjusted to ensure that the average heat generation rate of the drift segment is the same as that of the repository as a whole.

Figure 15 shows the interaction of repository-scale, fuel assembly-scale, and waste packagescale representations.

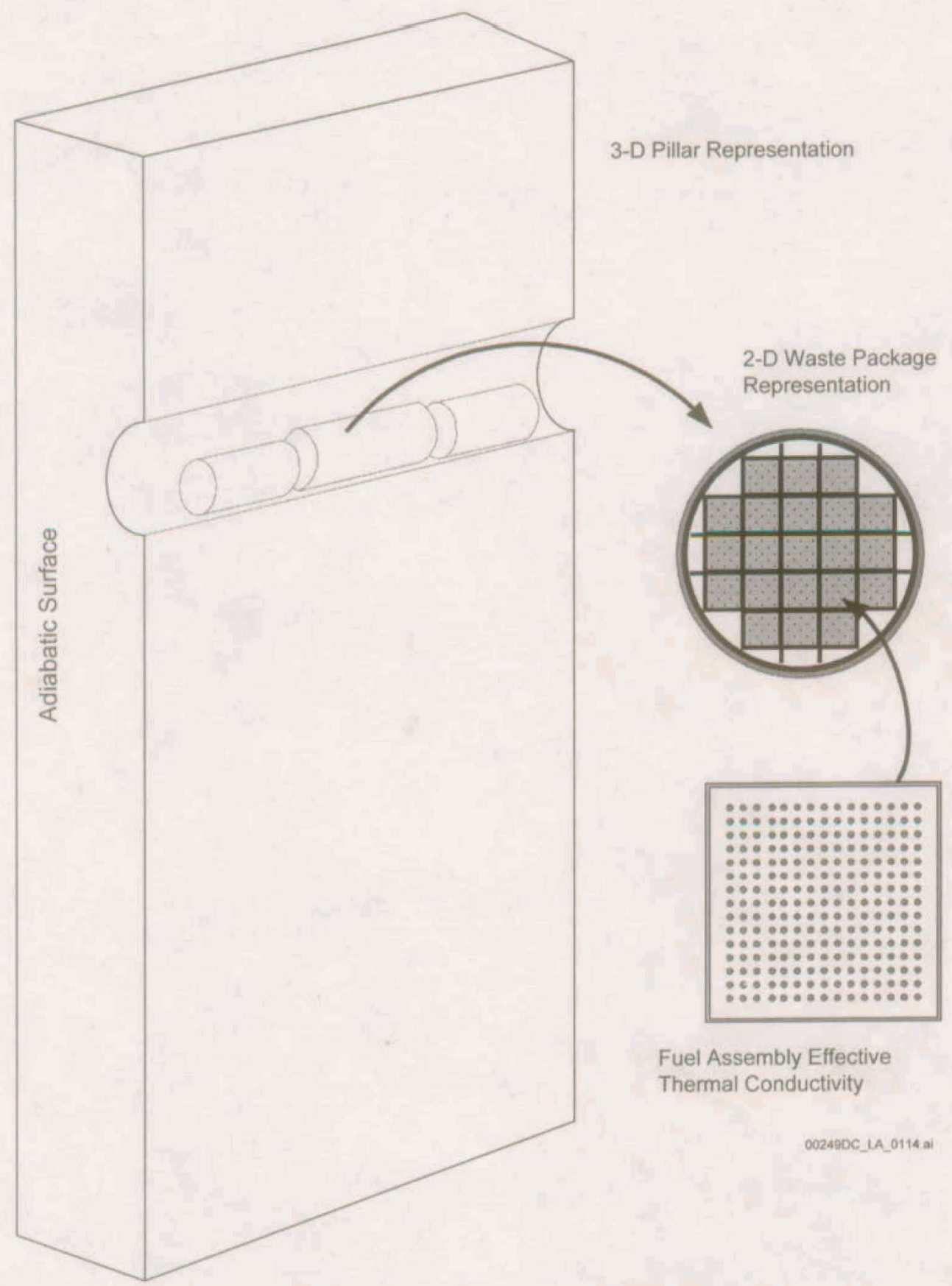

Figure 15. Multi-Scale Thermal Analysis Representation 


\subsubsection{Thermal Calculatioins of Fire Events}

Parametric calculations are performed using a 2-D ANSYS simulation of a waste package. Duration and temperature of the fire conditions are varied.

\subsubsection{Thermal Calculations in Surface Facilities}

Calculations are performed using a 2-D ANSYS simulation of a waste package in surface facilities. Thermal response is calculated for waste packages located on the transporter, in the weld cell, and other surface facilities.

\subsection{SHIELDING DESIGN}

The purpose of shielding analyses is to evaluate the effects of ionizing radiation on personnel, equipment, and materials. For waste package shielding, gamma rays and neutrons emitting from the commercial SNF or defense HLW are the primary radiation sources. During normal operations at the repository, loading and handling of the waste packages will be carried out remotely to avoid exposure of personnel to harmful radiation levels. Shielding analyses are performed primarily to assess radiation effects to materials and equipment.

Because waste packages are required to contain waste for thousands of years, the waste package barriers must reduce radiation levels at the waste package surface such that radiolysis-enhanced corrosion under aqueous conditions is negligible. Shielding analyses are hence carried out to determine radiation dose on the waste package surface, in order to evaluate the consequence of the radiolytically induced corrosion. Shielding evaluations are also performed to determine radiation exposure to equipment during welding of the waste package closure lids. Monitoring and control equipment, such as the welding heads and cameras, will be relatively close to the radiation sources. The results of the shielding evaluation will be used to quantify the shielding necessary for equipment to function properly at a given location for a required period of time.

In the event of emergency situations, personnel access in the proximity of the waste packages. may be required. Shielding analyses provide an evaluation of the radiation environment surrounding the waste packages, assuring safety of the personnel.

\subsubsection{Computational Methods and Tools}

Shielding analyses concern attenuation of neutrons and gamma rays through materials. The radiation dose rates outside a waste package are determined by solving the Boltzmann equation for radiation transport, which governs the behavior of the radiation particles in a material. Two methods for solving the Boltzmann transport equation for shielding applications have received the most development and have been used extensively for radiation shielding problems. They are the discrete-ordinates method and the Monte Carlo method. The computational tools used for waste package shielding analyses rely on these two methods.

\subsubsection{Monte Carlo Method}

The Monte Carlo method obtains radiation doses for shielding problems by employing a stochastic process to solve the Boltzmann transport equation. Using random variables, an 
"analog" Monte Carlo method simulates the histories of individual particles through the geometry (the "random-walk" process) and then analyzes these particle histories to derive the desired responses, such as flux density and dose rate. One particle history includes the birth of a particle at its source, its "random walk" through the transporting medium as it undergoes various scattering interactions, and ultimately the death of the particle, which terminates the history. A death can occur when the particle is absorbed, leaves the system, or loses significance owing to other factors.

For waste package shielding analyses, the analog Monte Carlo method is inadequate and inefficient in calculating radiation responses with acceptable accuracy because the events of interest are usually very rare. From the shielding point of view, the particles that escape the waste package are of primary interest for radiation dose evaluation. However, the probability of recording such an event in a Monte Carlo calculation is extremely low (less than $1 \times 10^{-5}$ ), and an unacceptably large number of histories is required to obtain acceptable results. For this reason, variance-reduction techniques must be employed for Monte Carlo shielding analyses. Variance-reduction techniques are procedures for altering the analog Monte Carlo process so as to reduce the variance of the calculated results. They are also known as "importance sampling" or "biasing techniques." The natural distributions in the "random walk" are modified by some importance function, and the particle statistical weights are adjusted from the analog value of unity to remove the bias. The purpose of variance-reduction techniques in Monte Carlo shielding analyses is to improve the efficiency of a calculation by reducing the variance of the results without increasing the computing time. The objective is to maintain a reasonable particle population in the primary regions of interest and, at the same time, control the fluctuation of statistical weight of the particles.

\subsubsection{Discrete-Ordinates Method}

The discrete-ordinates, or $S_{n}$, method solves the Boltzmann transport equation by using deterministic numerical techniques. The $S_{n}$ method is based on expressing the continuous form of the Boltzmann transport equation in terms of discrete values of the space, energy, and angle variables. Whereas the continuous transport equation represents particle balance over differential intervals, the discrete-ordinates formulation represents particle balance over finite intervals. The spatial variables are expressed as finite intervals; the angular variables are specified in terms of a finite number of discrete directions and corresponding weights, representing solid angles, and the energy domain is divided into a finite number of ranges called energy groups.

\subsubsection{MCNP}

MCNP (Briesmeister 1997 [DIRS 103897]) is a general-purpose Monte Carlo computer code for neutron, photon, electron, or coupled neutron/photon/electron transport. It is capable of calculating eigenvalues for critical systems and performing fixed-source (shielding) calculations to obtain radiation doses. For waste package design, MCNP is used for criticality and shielding calculations. The code allows a detailed geometric representation of the system being analyzed. MCNP uses continuous-energy nuclear and atomic data libraries. The MCNP package provides nuclear data tables derived from the Evaluated Nuclear Data File system, the Evaluated Nuclear Data Library and the Activation Library from Lawrence Livermore National Laboratory, and 
evaluations from the Applied Nuclear Science (T-2) Group at Los Alamos (Briesmeister 1997 [DIRS 103897], p. 1-4). MCNP evaluates the secondary gamma radiation in a coupled neutron/photon transport as well as the Bremsstrahlung radiation produced by the electrons generated in the photon transport.

Because of its versatility, MCNP is extensively used in dose rate evaluations for the waste packages. MCNP also serves to confirm the validity of the homogenized SNF assemblies used in SAS1 calculations (Section 6.4.1.4) and to determine the effect of source geometry on the waste package surface doses. The input specification in the MCNP dose rate calculations represents a conservative or equivalent treatment of the system being analyzed. MCNP applicability to the dose rate evaluations for the waste packages requires the following code features and calculational approaches:

- Separate photon and neutron transport calculations.

- A coupled neutron/photon transport calculation when capture gamma rays (photons created as a result of a neutron being captured by a nucleus) significantly contribute to the total dose rate.

- Photon or neutron surface flux tally specification.

- Dose function specification, which consists of flux-to-dose conversion factors provided in the MCNP manual (Briesmeister 1997 [DIRS 103897], pp. H-5 and H-6). These fluxto-dose conversion factors are extracted from ANSI/ANS-6.1.1-1977 [DIRS 107016].

- Photon interaction information.

- Available neutron continuous-energy cross-section tables, preferably those for neutron interaction cross-sections with elements of the attenuating medium.

- Contents of the source regions homogenized inside region volumes. The commercial SNF consists of four source regions: bottom end-fitting, active fuel, plenum, and top endfitting regions. Studies of the effect of source geometry on the waste package surface dose rates have shown that the homogenization of the assembly contents and source inside the assembly regions gives practically the same waste package surface dose rates as does the detailed geometric representation (CRWMS M\&O 1998 [DIRS 102134], pp. 22 to 26 ).

- Uniform volume source distribution specifications in each source region of the uncanistered commercial fuel and defense HLW packages. Because the radiation source generation method assumes a uniform burnup within the active fuel region, an axial peaking factor is used for photon and neutron source intensity in the active fuel region to conservatively account for the maximum values of the actual axial source distributions.

- The default implicit neutron and photon capture and cell importance based on the MCNP manual recommendations (Briesmeister 1997 [DIRS 103897], p. 2-121) as variance 
reduction techniques. The shielding analysis for the waste packages does not require extensive use of variance reduction techniques.

\subsubsection{SAS1}

SAS1 is a one-dimensional discrete-ordinates shielding calculation sequence using simplified input. SAS1 is a module of the SCALE computer code system (NRC 1997 [DIRS 122675]) consisting of three processes: (1) preparation of the multi-group cross-section information and mixing table used for the shielding calculation, (2) execution of a one-dimensional radiation transport analysis, and (3) calculation of dose rates outside the waste package. The default neutron and photon-to-dose rate conversion factors used in SAS1 are extracted from ANSI/ANS-6.1.1-1977 [DIRS 107016]. For waste package shielding analyses, SAS1 provides an effective and efficient tool for evaluating the radiation level on and beyond the radial outer surface of the waste package.

SAS1 represents a waste package as an infinite cylinder with a homogenized fuel region in the center, enclosed by the inner vessel and outer corrosion barrier. The homogenized fuel region consists of the waste form, neutron absorber plates, thermal shunts, and other structural members. Because the length of a waste package is approximately three times the diameter, the infinite cylinder representation of the waste package is assumed (Section 5.4.9) and has been shown to yield accurate dose results for the radial direction. SAS1 can also be used to estimate the radiation dose rate in the emplacement drift because the internals of the waste package are approximated as an infinite cylinder.

SAS1 has been applied in parametric studies to evaluate the effect of shielding materials on the waste package and to examine the individual dose rates due to neutrons, gamma rays, and capture gamma rays as functions of time for waste forms with different initial enrichment and burnup characteristics.

\subsubsection{Description of Pertinent Analyses}

Shielding analyses are performed for several waste package designs including: the 21-PWR waste package, 44-BWR waste package, and the 5-DHLW/DOE SNF codisposal waste package. For all waste package designs, radiation dose rates in the axial and radial directions are determined on segments of the waste package surfaces. The results of the shielding calculations allow an estimation of the average operation time of welding equipment, radiolysis-induced corrosion, and the radiation environment outside the waste packages for personnel access.

\subsubsection{21-PWR and 44-BWR Waste Package Analyses}

For the 21-PWR and 44-BWR waste packages, neutrons are present only in the active fuel, but photons emit from the active fuel, plenum, top-end-fitting, and bottom-end-fitting regions. Shielding calculations for the commercial waste packages must include all sources in order to compute the dose rates of the waste packages. Dose rates due to average, design basis, and maximum sources are computed. 


\subsubsection{5-DHLW/DOE Spent Nuclear Fuel Waste Package Analyses}

The 5-DHLW/DOE SNF waste package contains five HLW glass canisters and a central DOE SNF canister. The DOE SNF canister holds one of the many DOE-owned waste forms (BSC 2003 [DIRS 167273], Table 5). The HLW glass canister from the Savannah River Site is used in the shielding analyses because it has the maximum intensity among the HLW glass canisters (CRWMS M\&O 2000 [DIRS 151947], pp. V-1 to V-7 and pp. VI-1 to VI-7).

\subsection{CRITICALITY DESIGN}

The purpose of the waste package criticality analyses is to ensure that the waste package designs will fulfill their safety and waste isolation functions for nuclear criticality.

\subsubsection{Computational Methods and Tools}

The criticality analysis methodology for the repository is described in two reports. The process for analyzing the potential for criticality during preclosure is described in the Preclosure Criticality Analysis Process Report (BSC 2004 [DIRS 169590]). This report describes how criticality safety analyses are performed for various configurations of waste in and out of waste packages that could occur during preclosure as a result of normal operations or event sequences. The methodology for analyzing the potential for criticality during postclosure is described in the Disposal Criticality Analysis Methodology Topical Report (YMP 2003 [DIRS 165505]). This report describes how criticality and consequence analyses are performed for various configurations of waste in and out of waste packages in the repository during postclosure. The preclosure and postclosure methodologies are consistent with the NRC approach of supporting risk-informed performance-based regulation as required by 10 CFR Part 63 [DIRS 156605]. Details of the modeling approaches supporting the preclosure and postclosure analyses are documented in five model reports: Errata for Isotopic Model Report for Commercial SNF Burnup Credit (BSC 2003 [DIRS 168037]), Criticality Model Report (BSC 2003 [DIRS 165733]), Configuration Generator Model for In-Package Criticality (BSC 2003 [DIRS 165629]), Geochemistry Model Validation Report: External Accumulation Model (BSC 2001 [DIRS 156324]), and Geochemistry Model Validation Report: Material Degradation and Release Model (BSC 2001 [DIRS 156790]). The first two model reports support preclosure and postclosure analyses, whereas the last three reports support only postclosure analyses.

\subsubsection{Description of Pertinent Analyses}

Preclosure criticality analyses were performed for each waste package design configuration (e.g., 21-PWR Absorber Plate, 44-BWR Absorber Plate, 5-DHLW Codisposal short) during normal operations and for event sequences that could lead to a criticality event. Out-of-package evaluations for all waste forms and in-package evaluations for DOE SNF and HLW use the most reactive fuel state (i.e., fresh fuel assumption with no burnup credit for nonbreeder reactor fuel or calculated most reactive state for breeder reactor fuel). In-package evaluations for commercial SNF include credit for burnup similar to the postclosure methodology (YMP 2003 [DIRS 165505]). The design configuration analyses for commercial SNF packages produce loading curves (i.e., the minimum burnup) as a function of initial ${ }^{235} \mathrm{U}$ enrichment, at which assemblies may be loaded in a given waste package design configuration, and still keep the 
effective neutron multiplication factor $\left(\mathrm{k}_{\mathrm{eff}}\right)$ below the administrative safety limit. These analyses incorporate the methodology of Errata for Isotopic Model Report for Commercial SNF Burnup Credit (BSC 2003 [DIRS 168037]) and Criticality Model Report (BSC 2003 [DIRS 165733]). The design configuration analyses for DOE SNF and HLW waste packages verify that the waste package $k_{\text {eff }}$ remains below the administrative safety limit during normal operations. The event sequences analyses verify that the waste package $\mathrm{k}_{\text {eff }}$ remains below the administrative safety limit during credible event sequences.

Postclosure criticality analyses are also performed for each waste package design configuration. These analyses evaluate the combined system of waste form, waste package, engineered barrier, and repository for effectiveness in limiting the potential for criticality, the consequences of criticality, and the affect of any consequences on the regulatory performance objectives of the repository (YMP 2003 [DIRS 165505], Abstract). Analyses are performed for waste packages in a variety of hypothetical conditions ranging from geometrically intact through various stages of degradation of the package and contents (e.g., basket intact but fuel degraded or basket and fuel degraded). The analyses of degraded conditions rely on information from all the model reports described in Section 6.5.1. In addition to various stages of degradation, conditions such as partial or full flooding and partial or full reflection by tuff and water are considered. Furthermore, the effects of seismic rockfall and volcanic disruptive events are considered. Because the events and conditions that may occur cannot be predicted, the determination of possible criticality scenarios, analyses of those scenarios, and the resulting consequences are evaluated using a risk-informed, performance-based methodology (YMP 2003 [DIRS 165505]). The references listed in Section 6.5.1 provide technical details of the criticality analyses.

\section{SUMMARY}

No developed data were created in this report. This report describes the methodology used to design waste packages, emplacement pallets, and drip shields; however, it does not make recommendations regarding the final design of waste packages or the repository. The accounting of uncertainties in the methodology falls into one of two categories. For most, parameters are selected that produce net conservative results. These require no additional treatment. For a few, comparison against benchmarks, either experimental or high-order computational methods, must be performed. Comparisons against benchmarks are to be addressed in later revisions of this document. Because this is a design methodology, subsequent use is restricted only to the extent that such use would invalidate the theory underlying the particular application. 


\section{INPUTS AND REFERENCES}

\subsection{DOCUMENTS CITED}

Allegheny Ludlum. 1999. "Technical Data Blue Sheet, Stainless Steels, Chromium-NickelMolybdenum, Types 316 (S31600), 316L (S31603), 317 (S31700), 317L (S31703)." Pittsburgh, Pennsylvania: Allegheny Ludlum Corporation. Accessed July 31, 2000. TIC: 248631. [DIRS 151409]

http://www.alleghenytechnologies.com/ludlum/ pages/products/t316_317.pdf

Allison, J.M. 2004. "Request for Referenceable Information on High-Level Waste (HLW) Radionuclide Inventories in Support of Preparation of the Yucca Mountain Project License Application (Your Letter, JCP-0445, 1/28/04)." Memorandum from J.M. Allison (DOE/SR) to J. Arthur, III (OCRWM), February 26, 2004, 0303040661, with attachment. ACC: MOL.20040317.0265. [DIRS 168734]

Arthur, W.J., III. 2003. "Level 2 Directed Baseline Change (DOE/RW-0600, Rev. 0)." Letter from W.J. Arthur, III (DOE/ORD) to J.T. Mitchell (BSC), March 6, 2003, OPC\&M:JCD-0610, 0307036365, with enclosure. ACC: DOC.20030528.0004. [DIRS 164983]

ASHRAE (American Society of Heating, Refrigerating \& Air-Conditioning Engineer) 2001. 2001 ASHRAE Handbook, Fundamentals. Inch-Pound Edition. Atlanta, Georgia: American Society of Heating, Refrigerating and Air-Conditioning Engineers. TIC: 249910. [DIRS 157789]

ASM (American Society for Metals) 1961. Properties and Selection of Metals. Volume 1 of Metals Handbook. 8th Edition. Lyman, T., ed. Pages 506-507. Metals Park, Ohio: American Society for Metals. TIC: 252319. [DIRS 158132]

ASM (American Society for Metals) 1978. Properties and Selection: Irons and Steels. Volume 1 of Metals Handbook. 9th Edition. Bardes, B.P., ed. Metals Park, Ohio: American Society for Metals. TIC: 209799. [DIRS 102018]

ASM 1980. Properties and Selection: Stainless Steels, Tool Materials and Special-Purpose Metals. Volume 3 of Metals Handbook. 9th Edition. Benjamin, D., ed. Metals Park, Ohio: American Society for Metals. TIC: 209801. [DIRS 104317]

ASM International 1990. Properties and Selection: Nonferrous Alloys and Special-Purpose Materials. Volume 2 of ASM Handbook. Formerly 10th Edition, Metals Handbook. 5th Printing 1998. [Materials Park, Ohio]: ASM International. TIC: 241059. [DIRS 141615]

ASM International. 1987. Corrosion. Volume 13 of Metals Handbook. 9th Edition. Metals Park, Ohio: ASM International. TIC: 209807. [DIRS 103753]

Avallone, E.A. and Baumeister, T., III, eds. 1987. Marks' Standard Handbook for Mechanical Engineers. 9th Edition. New York, New York: McGraw-Hill. TIC: 206891. [DIRS 103508] 
Beer, F.P. and Johnston, E.R., Jr. 1977. Vector Mechanics for Engineers, Statics. 3rd Edition. New York, New York: McGraw-Hill Book Company. TIC: 247391. [DIRS 145138]

Bowles, J.E. 1980. Structural Steel Design. New York, New York: McGraw-Hill. TIC: 249182. [DIRS 153409]

Bowman, S.M.; Hermann, O.W.; and Brady, M.C. 1995. Sequoyah Unit 2 Cycle 3. Volume 2 of Scale-4 Analysis of Pressurized Water Reactor Critical Configurations. ORNL/TM-12294/V2. Oak Ridge, Tennessee: Oak Ridge National Laboratory. TIC: 244397. [DIRS 123796]

Boyer, H.E., ed. 2000. Atlas of Stress-Strain Curves. Metals Park, Ohio: ASM International. TIC: 248901. [DIRS 152656]

Briesmeister, J.F., ed. 1997. MCNP-A General Monte Carlo N-Particle Transport Code. LA12625-M, Version 4B. Los Alamos, New Mexico: Los Alamos National Laboratory. ACC: MOL.19980624.0328. [DIRS 103897]

BSC (Bechtel SAIC Company) 2001. Geochemistry Model Validation Report: External Accumulation Model. ANL-EBS-GS-000002 REV 00. Las Vegas, Nevada: Bechtel SAIC Company. ACC: MOL.20011008.0005. [DIRS 156324]

BSC (Bechtel SAIC Company) 2001. Geochemistry Model Validation Report: Material Degradation and Release Model. ANL-EBS-GS-000001 REV 00. Las Vegas, Nevada: Bechtel SAIC Company. ACC: MOL.20011017.0091. [DIRS 156790]

BSC (Bechtel SAIC Company) 2001. Statement of Work for DOE-Office of Civilian Radioactive Waste Management, Technical Assistance on Melt-Dilute Criticality and Shielding Analyses, Revision 2, May 30, 2001. Las Vegas, Nevada: Bechtel SAIC Company. ACC: MOL.20010619.0626. [DIRS 155245]

BSC (Bechtel SAIC Company) 2002. Software Code: ANSYS. V5.6.2. HP-UX 11.00. Software Tracking Number 10364-5.6.2-01. [DIRS 159357]

BSC (Bechtel SAIC Company) 2002. Software Code: DriftFlow. V1.0. Windows 2000. Software Tracking Number 10722-1.0-00. [DIRS 163090]

BSC (Bechtel SAIC Company) 2002. Software Code: LS-DYNA. V960.1106. HP9000. Software Tracking Number 10300-960.1106-00. [DIRS 158898]

BSC 2003. BSC Position on the Use of the ASME Boiler and Pressure Vessel Code for the Yucca Mountain Waste Packages. 000-30R-WIS0-00200-000-000. Las Vegas, Nevada: Bechtel SAIC Company. ACC: MOL.20030909.0054. [DIRS 165058]

BSC 2003. BWR Source Term Generation and Evaluation. 000-00C-MGR0-00200-000-00A. Las Vegas, Nevada: Bechtel SAIC Company. ACC: ENG.20030723.0001. [DIRS 164364] 
BSC (Bechtel SAIC Company) 2003. Configuration Generator Model for In-Package Criticality. MDL-EBS-NU-000001 REV 01 ICN 01. Las Vegas, Nevada: Bechtel SAIC Company. ACC: DOC.20030908.0004. [DIRS 165629]

BSC 2003. Criticality Model Report. MDL-EBS-NU-000003 REV 02. Las Vegas, Nevada: Bechtel SAIC Company. ACC: DOC.20031013.0002. [DIRS 165733]

BSC 2003. Determination of PWR Spent Nuclear Fuel Assembly Effective Thermal Conductivity. 000-00C-WIS0-00200-000-00A. Las Vegas, Nevada: Bechtel SAIC Company. ACC: ENG.20030324.0003. [DIRS 162416]

BSC (Bechtel SAIC Company) 2003. Dose Rate Calculation for 44-BWR Waste Package. 00000C-DSU0-02300-000-00A. Las Vegas, Nevada: Bechtel SAIC Company. ACC: ENG.20030909.0003. [DIRS166596]

BSC (Bechtel SAIC Company) 2003. Errata for Isotopic Model Report for Commercial SNF Burnup Credit. MDL-DSU-NU-000001 REV 00 ICN 01. Las Vegas, Nevada: Bechtel SAIC Company. ACC: DOC.20030904.0003; DOC.20031013.0004. [DIRS 168037]

BSC (Bechtel SAIC Company) 2003. Repository Twelve Waste Package Segment Thermal Calculation. 800-00C-WIS0-00100-000-00A. Las Vegas, Nevada: Bechtel SAIC Company. ACC: ENG.20030915.0003. [DIRS 164726]

BSC (Bechtel SAIC Company) 2003. Preliminary Categorization of Event Sequences for License Application. CAL-MGR-RL-000002 REV 00A. Las Vegas, Nevada: Bechtel SAIC Company. ACC: DOC.20030929.0010. [DIRS 164128]

BSC (Bechtel SAIC Company) 2003. Preliminary Nuclear Safety Design Bases for License Application. TDR-MGR-RL-000006 REV 00. Las Vegas, Nevada: Bechtel SAIC Company. ACC: DOC.20030930.0008. [DIRS165182]

BSC (Bechtel SAIC Company) 2003. Underground Layout Configuration. 800-P0C-MGR000100-000-00E. Las Vegas, Nevada: Bechtel SAIC Company. ACC: ENG.20031002.0007. [DIRS 165572]

BSC (Bechtel SAIC Company) 2004. DOE and Commercial Waste Package System Description Document. 000-3YD-DS00-00100-000-003. Las Vegas, Nevada: Bechtel SAIC Company. ACC: ENG.20040322.0008. [DIRS 167273]

BSC (Bechtel SAIC Company) 2004. Dose Rate Calculation for 21-PWR Waste Package. 00000C-DSU0-01800-000-00B. Las Vegas, Nevada: Bechtel SAIC Company. ACC:

ENG.20040607.0001. [DIRS 169593]

BSC (Bechtel SAIC Company) 2004. Drift Degradation Analysis. ANL-EBS-MD-000027 REV 02, Errata 1. Las Vegas, Nevada: Bechtel SAIC Company. ACC: DOC.20040325.0002; DOC.20030709.0003. [DIRS 168550] 
BSC (Bechtel SAIC Company) 2004. Inventory and Characteristics of Potential Repository Wastes. 000-00C-MGR0-01200-000-00A. Las Vegas, Nevada: Bechtel SAIC Company. ACC: ENG.20040517.0003. [DIRS 167441]

BSC (Bechtel SAIC Company) 2004. Naval Spent Nuclear Fuel Waste Package System Description Document. 000-3YD-DN00-00100-000-003. Las Vegas, Nevada: Bechtel SAIC Company. ACC: ENG.20040322.0006. [DIRS 167272]

BSC (Bechtel SAIC Company) 2004. PWR Source Term Generation and Evaluation. 000-00CMGR0-00100-000-00B. Las Vegas, Nevada: Bechtel SAIC Company. ACC: ENG.20040524.0007. [DIRS 169061]

BSC (Bechtel SAIC Company) 2004. Subsurface Facility Description Document. 800-3YDMGR0-00100-000-004. Las Vegas, Nevada: Bechtel SAIC Company. ACC: ENG.20040630.0006. [DIRS 170265]

BSC (Bechtel SAIC Company) 2004. Commercial SNF Waste Package Design Report. 00000C-DSU0-02800-000-00A. Las Vegas, Nevada: Bechtel SAIC Company. ACC:

ENG.20040315.0007. [DIRS 168217]

BSC (Bechtel SAIC Company) 2004. Errata for Seismic Consequence Abstraction. MDL-WISPA-000003 REV 0, Errata 1. Las Vegas, Nevada: Bechtel SAIC Company. ACC: DOC.20030818.0006; DOC.20040218.0002. [DIRS 167780]

BSC (Bechtel SAIC Company) 2004. HLW/DOE SNF Codisposal Waste Package Design Report. 000-00C-DS00-00600-000-00A. Las Vegas, Nevada: Bechtel SAIC Company. ACC: ENG.20040304.0003. (DIRS 166895)

BSC (Bechtel SAIC Company) 2004. Intact and Degraded Mode Criticality Calculations for the Codisposal of TMI-2 Spent Nuclear Fuel in a Waste Package. CAL-DSD-NU-000004 REV 00A. Las Vegas, Nevada: Bechtel SAIC Company. ACC: DOC.20040329.0002. [DIRS 168935 ]

BSC (Bechtel SAIC Company) 2004. Naval Waste Package Design Report. 000-00C-DNF000800-000-00A. Las Vegas, Nevada: Bechtel SAIC Company. ACC: ENG.20040316.0010. (DIRS 166894)

BSC (Bechtel SAIC Company) 2004. Waste Package Closure System Description Document. 100-3YD-HW00-00100-000-001. Las Vegas, Nevada: Bechtel SAIC Company. ACC: ENG.20040310.0021. [DIRS 167278]

BSC (Bechtel SAIC Company) 2004. Preclosure Criticality Analysis Process Report. TDREBS-NU-000004 Rev 03. Las Vegas, Nevada: Bechtel SAIC Company. ACC: DOC.20040524.0001. [DIRS 169590]

BSC (Bechtel SAIC Company) 2004. Steel Invert Structure-Emplacement Drifts. 800-SSCSSE0-00100-000-00A. Las Vegas, Nevada: Bechtel SAIC Company. ACC:

ENG.20040119.0012. [DIRS 166363] 
Canori, G.F. and Leitner, M.M. 2003. Project Requirements Document. TER-MGR-MD-000001 REV 02. Las Vegas, Nevada: Bechtel SAIC Company. ACC: DOC.20031222.0006. [DIRS $166275]$

Chun, R.; Witte, M.; and Schwartz, M. 1987. Dynamic Impact Effects on Spent Fuel Assemblies. UCID-21246. Livermore, California: Lawrence Livermore National Laboratory. ACC: HQX.19881020.0031. [DIRS 144357]

Creer, J.M.; Michener, T.E.; McKinnon, M.A.; Tanner, J.E.; Gilbert, E.R.; Goodman, R.L.; Dziadosz, D.A.; Moore, E.V.; McKay, H.S.; Batalo, D.P.; Schoonen, D.H.; Jensen, M.F.; and Mullen, C.K. 1987. The TN-24P PWR Spent-Fuel Storage Cask: Testing and Analyses. EPRI NP-5128. Palo Alto, California: Electric Power Research Institute. TIC: 228305. [DIRS 136937]

CRWMS M\&O (Civilian Radioactive Waste Management System) 1994. Thermal Evaluations of Waste Package Emplacement. BBA000000-01717-4200-00008 REV 00. Las Vegas, Nevada: CRWMS M\&O. ACC: MOL.19941007.0094. [DIRS 142611]

CRWMS M\&O 1995. Emplacement Scale Thermal Evaluations of Large and Small WP Designs. BB0000000-01717-0200-00009 REV 00. Las Vegas, Nevada: CRWMS M\&O. ACC: MOL.19960624.0097. [DIRS 106058]

CRWMS M\&O 1996. Determination of $7 x 7$ Rod Array BWR SNF Assembly Effective Thermal Conductivity. BBA000000-01717-0200-00015 REV 00. Las Vegas, Nevada: CRWMS M\&O.

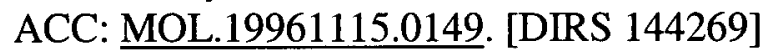

CRWMS M\&O 1996. Determination of 8x8 Rod Array BWR SNF Assembly Effective Thermal Conductivity. BBA000000-01717-0200-00017 REV 00. Las Vegas, Nevada: CRWMS M\&O. ACC: MOL.19961114.0090. [DIRS 144270]

CRWMS M\&O 1996. Determination of 9x9 Rod Array BWR SNF Assembly Effective Thermal Conductivity. BBA000000-01717-0200-00018 REV 00. Las Vegas, Nevada: CRWMS M\&O.

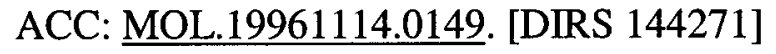

CRWMS M\&O 1996. Spent Nuclear Fuel Effective Thermal Conductivity Report. BBA00000001717-5705-00010 REV 00. Las Vegas, Nevada: CRWMS M\&O. ACC: MOL.19961202.0030. [DIRS 100215]

CRWMS M\&O 1997. Engineered Barrier System Performance Requirements Systems Study `Report. BB0000000-01717-5705-00001 REV 02. Las Vegas, Nevada: CRWMS M\&O. ACC: MOL.19970929.0316. [DIRS 101538]

CRWMS M\&O 1998. Calculation of the Effect of Source Geometry on the 21-PWR WP Dose Rates. BBAC00000-01717-0210-00004 REV 00. Las Vegas, Nevada: CRWMS M\&O. ACC: MOL.19990222.0059. [DIRS 102134]

CRWMS M\&O 1998. Software Code: MCNP. V4B2LV. HP, HPUX 9.07 and 10.20; PC, Windows 95; Sun, Solaris 2.6. Software Tracking Number 30033 V4B2LV. [DIRS 154060] 
CRWMS M\&O 1999. CRC Depletion Calculations for Quad Cities Unit 2. B00000000-017170210-00009 REV 01. Las Vegas, Nevada: CRWMS M\&O. ACC: MOL.19990929.0121. [DIRS 134650]

CRWMS M\&O 1999. Summary Report of Commercial Reactor Criticality Data for Quad Cities Unit 2. B00000000-01717-5705-00096 REV 01. Las Vegas, Nevada: CRWMS M\&O. ACC: MOL.19990917.0184. [DIRS 134660]

CRWMS M\&O 2000. Radionuclide Inventories for DOE SNF Waste Stream and Uranium/Thorium Carbide Fuels. CAL-MGR-NU-000003 REV 00. Las Vegas, Nevada: CRWMS M\&O. ACC: MOL.20000412.0764. [DIRS 153247]

CRWMS M\&O 2000. Software Code: SCALE. V4.4A. HP. Software Tracking Number 101294.4A-00. [DIRS 154394]

CRWMS M\&O 2000. Source Terms for Average DOE SNF Canisters. CAL-MGR-NU-000004 REV 00. Las Vegas, Nevada: CRWMS M\&O. ACC: MOL.20000627.0213. [DIRS 153345]

CRWMS M\&O 2000. Source Terms for HLW Glass Canisters. CAL-MGR-NU-000002 REV 01. Las Vegas, Nevada: CRWMS M\&O. ACC: MOL.20000823.0004. [DIRS 151947]

CRWMS M\&O 2000. Waste Packages and Source Terms for the Commercial 1999 Design Basis Waste Streams. CAL-MGR-MD-000001 REV 00. Las Vegas, Nevada: CRWMS M\&O.

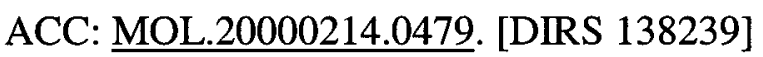

Dieter, G.E. 1976. Mechanical Metallurgy. 2nd Edition. Materials Science and Engineering Series. New York, New York: McGraw-Hill Book Company. TIC: 247879. [DIRS 118647]

DOE (U.S. Department of Energy) 1987. Appendix 2A. Physical Descriptions of LWR Fuel Assemblies. Volume 3 of Characteristics of Spent Fuel, High-Level Waste, and Other Radioactive Wastes Which May Require Long-Term Isolation. DOE/RW-0184. Washington, D.C.: U.S. Department of Energy, Office of Civilian Radioactive Waste Management. ACC: HQX.19880405.0024. [DIRS 132333]

DOE 1999. Fermi (U-Mo) Fuel Characteristics for Disposal Criticality Analysis.

DOE/SNF/REP-035, Rev. 0. Washington, D.C.: U.S. Department of Energy. TIC: 242461. [DIRS 104110]

DOE 1999. Shippingport LWBR (Th/U Oxide) Fuel Characteristics for Disposal Criticality Analysis. DOE/SNF/REP-051, Rev. 0. Washington, D.C.: U.S. Department of Energy, Office of Environmental Management. TIC: 245631. [DIRS 105007]

DOE 1999. Shippingport PWR (HEU Oxide) Fuel Characteristics for Disposal Criticality Analysis. DOE/SNF/REP-040, Rev. 0. Washington, D.C.: U.S. Department of Energy. TIC: 243528. [DIRS 104940] 
DOE 1999. TRIGA (UZrH) Fuel Characteristics for Disposal Criticality Analysis.

DOE/SNF/REP-048, Rev. 0. Washington, D.C.: U.S. Department of Energy. TIC: 244162. [DIRS 103891]

DOE 2000. N Reactor (U-Metal) Fuel Characteristics for Disposal Criticality Analysis. DOE/SNF/REP-056, Rev. 0. [Washington, D.C.]: U.S. Department of Energy, Office of Environmental Management. TIC: 247956. [DIRS 150095]

DOE 2002. Final Environmental Impact Statement for a Geologic Repository for the Disposal of Spent Nuclear Fuel and High-Level Radioactive Waste at Yucca Mountain, Nye County, Nevada. DOE/EIS-0250. Washington, D.C.: U.S. Department of Energy, Office of Civilian Radioactive Waste Management. ACC: MOL.20020524.0314; through; MOL.20020524.0320. [DIRS 155970]

DOE 2002. Waste Acceptance System Requirements Document. DOE/RW-0351, Rev. 4. Washington, D.C.: U.S. Department of Energy, Office of Civilian Radioactive Waste Management. ACC: MOL.20020326.0056. [DIRS 158873]

DOE 2003. Source Term Estimates for DOE Spent Nuclear Fuels. DOE/SNF/REP-078, Rev. 0. Idaho Falls, Idaho: U.S. Department of Energy, Idaho Operations Office. TIC: 254275. [DIRS 163377]

DOE 2004. Quality Assurance Requirements and Description. DOE/RW-0333P, Rev. 14. Washington, D.C.: U.S. Department of Energy, Office of Civilian Radioactive Waste Management. ACC: DOC.20040331.0004. [DIRS 168669]

EPRI (Electric Power Research Institute) 1989. Testing and Analyses of the TN-24P PWR SpentFuel Dry Storage Cask Loaded with Consolidated Fuel. EPRI NP-6191. Palo Alto, California: Electric Power Research Institute. TIC: 207047. [DIRS 101947]

Fluent, Inc. 2003. Software Code: FLUENT. V.6.0.12. HP, HP-UX 11.00. Software Tracking Number 10550-6.0.12-00. [DIRS 163001]

Framatome Cogema Fuels 1999. Operational Data - B\&W NSS. 51-5004349-01. Lynchburg, Virginia: Framatome Cogema Fuels. ACC: MOL.19990610.0201. [DIRS 146419]

Gartling, D.K.; Eaton, R.R.; and Thomas, R.K. 1981. Preliminary Thermal Analyses for a Nuclear Waste Repository in Tuff. SAND80-2813. Albuquerque, New Mexico: Sandia National Laboratories. ACC: HQS.19880517.2295. [DIRS 142640]

Goff, K.M. 1998. "Revision to Original INEEL Response to Yucca Mountain Site Characterization Office Data Call for High-Level Waste (Ref. Palmer and Benedict to Wichmann, July 2, 1997)." Letter from K.M. Goff (ANL-West) to M.B. Heiser (Lockheed), April 15, 1998, with attachment, "Modifications to Yucca Mountain Data Call." ACC: MOL.19990608.0032. [DIRS 104389] 
Hamel, W.F. 2003. "Waste Treatment and Immobilization Plant (WTP) High-Level Waste (HLW) Canister Production Estimates to Support Analyses by the Yucca Mountain Project." Memorandum from W.F. Hamel (DOE) to W.J. Taylor, June 26, 2003, with attachment. ACC:

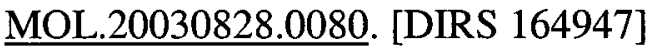

Haynes International. 1997. Hastelloy C-22 Alloy. Kokomo, Indiana: Haynes International. TIC: 238121. [DIRS 100896]

High Temp Metals. 2002. "17-4Ph Technical Data." Sylmar, California: High Temp Metals. Accessed March 19, 2002. TIC: 252170. [DIRS 158006] http://www.hightempmetals.com/hitemp17-4PHdata.htm

Inco Alloys International. 1988. Product Handbook. Huntington, West Virginia: Inco Alloys International. TIC: 239397. [DIRS 130835]

Incropera, F.P. and DeWitt, D.P. 1996. Introduction to Heat Transfer. 3rd Edition. New York, New York: John Wiley \& Sons. TIC: 241057. [DIRS 107784]

INEEL (Idaho National Engineering and Environmental Laboratory) 2002. FFTF (MOX) Fuel Characteristics for Disposal Criticality Analysis. DOE/SNF/REP-032, Rev. 1. Idaho Falls, Idaho: U.S. Department of Energy, Idaho National Operations Office. TIC: 252933. [DIRS 158820]

Jones, N. 1994. "Low Velocity Perforation of Metal Plates." Chapter 3 of Shock and Impact on Structures. Brebbia, C.A. and Sanchez-Galvez, V., eds. Billerica, Massachusetts: Computational Mechanics Publications. TIC: 247052. [DIRS 137700]

Jones, R.H. 1992. Spent Fuel Corrosion Product and Fuel Cleaning Assessment. Los Gatos,

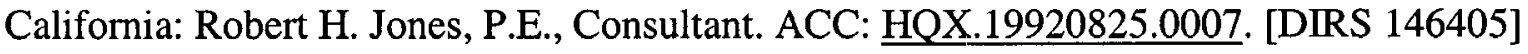

Kügler, A. 1997. Sheet and Plate for Nuclear Engineering, Bohler Neutronit A976. Houston, Texas: Bohler Bleche GmbH. TIC: 246410. [DIRS 134327]

Larsen, N.H.; Parkos, G.R.; and Raza, O. 1976. Core Design and Operating Data for Cycles 1 and 2 of Quad Cities 1. EPRI NP-240. Palo Alto, California: Electric Power Research Institute. TIC: 237267. [DIRS 146576]

Levy, I.S.; Chin, B.A.; Simonen, E.P.; Beyer, C.E.; Gilbert, E.R.; and Johnson, A.B., Jr. 1987. Recommended Temperature Limits for Dry Storage of Spent Light Water Reactor Zircaloy-Clad Fuel Rods in Inert Gas. PNL-6189. Richland, Washington: Pacific Northwest Laboratory. TIC: 231836. [DIRS 144349]

Lide, D.R., ed. 1995. CRC Handbook of Chemistry and Physics. 76th Edition. Boca Raton, Florida: CRC Press. TIC: 216194. [DIRS 101876] 
Ludwig, S.B. and Renier, J.P. 1989. Standard-and Extended-Burnup PWR and BWR Reactor Models for the ORIGEN2 Computer Code. ORNL/TM-11018. Oak Ridge, Tennessee: Oak Ridge National Laboratory. TIC: 203557. [DIRS 146398]

Luksic, A. 1989. Activation Measurements and Comparison with Calculations for Spent Fuel Assembly Hardware. Volume 1 of Spent Fuel Assembly Hardware: Characterization and 10 CFR 61 Classification for Waste Disposal. PNL-6906. Richland, Washington: Pacific Northwest Laboratory. ACC: NNA.19890926.0124. [DIRS 120506]

MathSoft 1998. Mathcad 8, User's Guide. Cambridge, Massachusetts: MathSoft. TIC: 242289. [DIRS 119437]

Meriam, J.L. and Kraige, L.G. 1987. Statics. Volume 1 of Engineering Mechanics. 2nd Edition. Pages 441, 443. New York, New York: John Wiley \& Sons. TIC: 241293. [DIRS 104306]

Minwalla, H.J. 2003. Project Design Criteria Document. 000-3DR-MGR0-00100-000-001. Las Vegas, Nevada: Bechtel SAIC Company. ACC: ENG.20030402.0001. [DIRS 161362]

Parrington, J.R.; Knox, H.D.; Breneman, S.L.; Baum, E.M.; and Feiner, F. 1996. Nuclides and Isotopes, Chart of the Nuclides. 15th Edition. San Jose, California: General Electric Company and KAPL, Inc. TIC: 233705. [DIRS 103896]

Picha, K.G., Jr. 1997. "Response to Repository Environmental Impact Statement Data Call for High-Level Waste." Memorandum from K.G. Picha, Jr. (DOE) to W. Dixon (YMSCO), September 5, 1997, with attachments. ACC: MOL.19970917.0273. [DIRS 104406]

Picha, K.G., Jr. 1998. "Clarification of High-Level Waste and Special Performance Assessment Required Data for Repository Environmental Impact Statement." Memorandum from K.G. Picha, Jr. (DOE) to K. Skipper (DOE/YMSCO), May 8, 1998, with attachments. ACC: MOL.19990610.0297. [DIRS 104407]

Picha, K.G., Jr. 1998. "Follow Up Response to Repository Environmental Impact Statement Data Call for High-Level Waste." Memorandum from K.G. Picha, Jr. (DOE) to W. Dixon (YMSCO), March 12, 1998, with attachment. ACC: MOL.19981006.0206. [DIRS 104413]

Plodinec, M.J. and Marra, S.L. 1994. Projected Radionuclide Inventories and Radiogenic Properties of the DWPF Product (U). WSRC-IM-91-116-3, Rev. 0. Aiken, South Carolina: Westinghouse Savannah River Company. TIC: 242337. [DIRS 101908]

Punatar, M.K. 2001. Summary Report of Commercial Reactor Criticality Data for Crystal River Unit 3. TDR-UDC-NU-000001 REV 02. Las Vegas, Nevada: Bechtel SAIC Company. ACC: MOL.20010702.0087. [DIRS 155635]

Sass, J.H.; Lachenbruch, A.H.; Dudley, W.W., Jr.; Priest, S.S.; and Munroe, R.J. 1988. Temperature, Thermal Conductivity, and Heat Flow Near Yucca Mountain, Nevada: Some Tectonic and Hydrologic Implications. Open-File Report 87-649. [Denver, Colorado]: U.S. Geological Survey. TIC: 203195. [DIRS 100644] 
Siddoway, D.W. 2003. Project Functional and Operational Requirements. TDR-MGR-ME000003 REV 01. Las Vegas, Nevada: Bechtel SAIC Company. ACC: ENG.20030630.0001. [DIRS 163904]

Siegel, R. and Howell, J.R. 1992. Thermal Radiation Heat Transfer. 3rd Edition. Washington, D.C.: Taylor \& Francis. TIC: $\underline{236759}$. [DIRS 100687]

Stout, R.B. and Leider, H.R., eds. 1997. Waste Form Characteristics Report Revision 1. UCRLID-108314. Version 1.2. Livermore, California: Lawrence Livermore National Laboratory. ACC: MOL.19980512.0133. [DIRS 100419]

Taylor, L.L. 2001. Fort Saint Vrain HTGR (Th/U Carbide) Fuel Characteristics for Disposal Criticality Analysis. DOE/SNF/REP-060, Rev. 0. [Washington, D.C.]: U.S. Department of Energy, Office of Environmental Management. TIC: 249783. [DIRS 154726]

Taylor, W.J. 1997. "Response to Clarification Data for the Repository Environmental Impact Statement (EIS) Data Call Memorandum Dated October 3, 1997." Memorandum from W.J. Taylor (DOE) to K.G. Picha, Jr. (Office of Waste Management), November 17, 1997, EIS:ARGEN-35721, with attachments. ACC: MOL.19990610.0295. [DIRS 104476]

TIMET. 2000. "Timetal 6-4, 6-4 ELI, 6-4-.1Ru Medium to High Strength General-Purpose Alloys." Denver, Colorado: Titanium Metals Corporation. Accessed August 26, 2002. TIC: 253102. [DIRS 160688]

http://www.timet.com/pdfs/6-4.pdf

Todreas, N.E. and Kazimi, M.S. 1990. Nuclear Systems I, Thermal Hydraulic Fundamentals. New York, New York: Hemisphere Publishing. TIC: 226511. [DIRS 107735]

Wooton, R.O. and Epstein, H.M. 1963. Heat Transfer from a Parallel Rod Fuel Element in a Shipping Container. Columbus, Ohio: Battelle Memorial Institute. TIC: 236802. [DIRS 144379]

WVNS (West Valley Nuclear Services Company) 1998. Waste Form Qualification Report (WQR), West Valley Demonstration Project. WVDP-186. West Valley, New York: West Valley Nuclear Services Company. TIC: 242094. [DIRS 103500]

WVNS (West Valley Nuclear Services) 2003. "Estimated Radionuclide Inventory." Addendum 2 of WVDP Waste Form Qualification Report - Waste Form Specifications, Radionuclide Inventory Specification. WVDP-186, Rev. 0. [West Valley, New York]: West Valley Nuclear Services. ACC: $\underline{\text { MOL.20040329.0112. [168661] }}$

YMP (Yucca Mountain Site Characterization Project) 2003. Disposal Criticality Analysis Methodology Topical Report. YMP/TR-004Q, Rev. 02. Las Vegas, Nevada: Yucca Mountain Site Characterization Office. ACC: DOC.20031110.0005. [DIRS 165505] 


\subsection{CODES, STANDARDS, REGULATIONS, AND PROCEDURES}

10 CFR 63. Energy: Disposal of High-Level Radioactive Wastes in a Geologic Repository at Yucca Mountain, Nevada. Readily available. [DIRS 156605]

10 CFR 961. Energy: Standard Contract for Disposal of Spent Nuclear Fuel and/or High-Level Radioactive Waste. Readily available.

ANSI N14.6-1993. American National Standard for Radioactive Materials - Special Lifting Devices for Shipping Containers Weighing 10000 Pounds (4500 kg) or More. New York, New York: American National Standards Institute. TIC: 236261. [DIRS 102016]

ANSIANS-6.1.1-1977. Neutron and Gamma-Ray Flux-to-Dose-Rate Factors. La Grange Park, Illinois: American Nuclear Society. TIC: 239401. [DIRS 107016]

AP-3.11Q, Rev. 4 ICN 1. Technical Reports. Washington, D.C.: U.S. Department of Energy, Office of Civilian Radioactive Waste Management. ACC: DOC.20040217.0001.

ASME (American Society of Mechanical Engineers) 2001. 2001 ASME Boiler and Pressure Vessel Code (includes 2002 addenda). New York, New York: American Society of Mechanical Engineers. TIC: 251425. [DIRS 158115]

ASTM A 240/A 240M-97a. 1997. Standard Specification for Heat-Resisting Chromium and Chromium-Nickel Stainless Steel Plate, Sheet, and Strip for Pressure Vessels. West

Conshohocken, Pennsylvania: American Society for Testing and Materials. TIC: 239431. [DIRS 102769]

ASTM A 887-89 (Reapproved 2000). 2000. Standard Specification for Borated Stainless Steel Plate, Sheet, and Strip for Nuclear Application. West Conshohocken, Pennsylvania: American Society for Testing and Materials. TIC: 249544. [DIRS 154062]

ASTM B 811-90. 1991. Standard Specification for Wrought Zirconium Alloy Seamless Tubes for Nuclear Reactor Fuel Cladding. Philadelphia, Pennsylvania: American Society for Testing and Materials. TIC: 239780. [DIRS 131753]

ASTM G 1-90 (Reapproved 1999). 1999. Standard Practice for Preparing, Cleaning, and Evaluating Corrosion Test Specimens. West Conshohocken, Pennsylvania: American Society for Testing and Materials. TIC: 238771. [DIRS 103515]

Gerhard, M.A.; Trummer, D.J.; Johnson, G.L.; and Mok, G.C. 1992. SCANS (Shipping Cask Analysis System) A Microcomputer Based Analysis System for Shipping Cask Design Review, User's Manual to Version 2 a (Including Program Reference). NUREG/CR-4554. Volume 1, Rev. 1. Washington, D.C.: U.S. Nuclear Regulatory Commission. TIC: 236412. [DIRS 145318] 
Hagrman, D.L.; Reymann, G.A.; and Mason, R.E., eds. 1981. MATPRO - Version 11 (Revision 2), A Handbook of Materials Properties for Use in the Analysis of Light Water Reactor Fuel Rod Behavior. NUREG/CR-0497, Rev. 2. Washington, D.C.: U.S. Nuclear Regulatory Commission. TIC: 209823. [DIRS 103522]

Hechmer, J.L. and Hollinger, G.L. 1998. 3D Stress Criteria Guidelines for Application. WRC Bulletin 429. New York, New York: Welding Research Council. TIC: 254432. [DIRS 166147]

Hermann, O.W.; Parks, C.V.; and Renier, J.P. 1994. Technical Support for a Proposed Decay Heat Guide Using SAS2H/ORIGEN-S Data. NUREG/CR-5625. Washington, D.C.: U.S. Nuclear Regulatory Commission. TIC: 212756. [DIRS 154045]

Holman, W.R. and Langland, R.T. 1981. Recommendations for Protecting Against Failure by Brittle Fracture in Ferritic Steel Shipping Containers up to Four Inches Thick. NUREG/CR1815. Washington, D.C.: U.S. Nuclear Regulatory Commission. TIC: 234364. [DIRS 145192]

Lee, A.S. and Bumpas, S.E. 1995. Buckling Analysis of Spent Fuel Basket. NUREG/CR-6322. Washington, D.C.: U.S. Nuclear Regulatory Commission. TIC: 232183. [DIRS 154068]

NRC (U.S. Nuclear Regulatory Commission) 1980. Control of Heavy Loads at Nuclear Power Plants. NUREG-0612. Washington, D.C.: U.S. Nuclear Regulatory Commission. TIC: 209017. [DIRS 104939]

NRC 1997. SCALE, RSIC Computer Code Collection (CCC-545). NUREG/CR-0200, Rev. 5. Washington, D.C.: U.S. Nuclear Regulatory Commission. TIC: 235920. [DIRS 122675]

NRC 1997. Standard Review Plan for Dry Cask Storage Systems. NUREG-1536. Washington, D.C.: U.S. Nuclear Regulatory Commission. ACC: MOL.20010724.0307. [DIRS 101903]

NRC 2000. Standard Review Plan for Spent Fuel Dry Storage Facilities. NUREG-1567. Washington, D.C.: U.S. Nuclear Regulatory Commission. TIC: 247929. [DIRS 149756]

NRC 2000. Standard Review Plan for Transportation Packages for Spent Nuclear Fuel. NUREG-1617. Washington, D.C.: U.S. Nuclear Regulatory Commission. TIC: 249470. [DIRS 154000]

NRC 2003. Yucca Mountain Review Plan, Final Report. NUREG-1804, Rev. 2. Washington, D.C.: U.S. Nuclear Regulatory Commission, Office of Nuclear Material Safety and Safeguards. TIC: 254568. [DIRS 163274]

Nuclear Waste Policy Amendments Act of 1987. Public Law No. 100-203, 101 Stat. 1330.

Readily available. [DIRS 100016]

Rack, H.J. and Knorovsky, G.A. 1978. An Assessment of Stress-Strain Data Suitable for FiniteElement Elastic-Plastic Analysis of Shipping Containers. NUREG/CR-0481. Washington, D.C.: U.S. Nuclear Regulatory Commission. TIC: 247953. [DIRS 145185]

Witte, M.C.; Hovingh, J.; Mok, G.C.; Murty, S.S.; Chen, T.F.; and Fischer, L.E. 1998. Summary 
and Evaluation of Low-Velocity Impact Tests of Solid Steel Billet Onto Concrete Pads. NUREG/CR-6608. Washington, D.C.: U.S. Nuclear Regulatory Commission. TIC: 239732. [DIRS 136115]

\subsection{SOURCE DATA, LISTED BY DATA TRACKING NUMBER}

GS000483351030.003. Thermal Properties Measured 12/01/99 to 12/02/99 Using the Thermolink Soil Multimeter and Thermal Properties Sensor on Selected Potential Candidate Backfill Materials Used in the Engineered Barrier System. Submittal date: 11/09/2000. [DIRS 152932]

GS950308319213.002. Downhole Temperature Data for Wells Near or At Yucca Mountain, Nevada. Submittal date: 03/08/1995. [DIRS 162990]

LL020603612251.015. Slow Strain Rate Test Generated Stress Corrosion Cracking Data.

Submittal date: 08/27/2002. [DIRS 160430]

MO0003RIB00079.000. Rock Mechanical Properties. Submittal date: 03/30/2000. [DIRS 148295]

MO0007RIB00077.000. In Situ Rock Conditions. Submittal date: 07/18/2000. [DIRS 154087]

MO0001SPADBE00.001. DOE Spent Nuclear Fuel Data Contained in DBEFEB99.XLS

Workbook. Submittal date: 01/04/2000. [DIRS 146362]

MO0301MWD3DE27.003. Results from 3Dec Nonlithophysal Rockfall Analyses with 10-7 Ground Motion Level. Submittal date: 01/23/2003. [DIRS 161536]

M09808RIB00041.000. Reference Information Base Data Item: Rock Geomechanical Properties. Submittal date: 08/05/1998. [DIRS 104850]

SN0303T0503102.008. Revised Thermal Conductivity of the Non-Repository Layers of Yucca Mountain. Submittal date: 03/19/2003. [DIRS 162401]

SN0404T0503102.011. Thermal Conductivity of the Potential Repository Horizon Rev 3. Submittal date: 04/27/2004. [DIRS 169129]

SN0307T0510902.003. Updated Heat Capacity of Yucca Mountain Stratigraphic Units. Submittal date: 07/15/2003. [DIRS 164196] 